MATHEMATICS OF COMPUTATION

Volume 78, Number 265, January 2009, Pages 441-483

S $0025-5718(08) 02105-4$

Article electronically published on May 2, 2008

\title{
NONTRIVIAL ELEMENTS OF SHA EXPLAINED THROUGH K3 SURFACES
}

\author{
ADAM LOGAN AND RONALD VAN LUIJK
}

\begin{abstract}
We present a new method to show that a principal homogeneous space of the Jacobian of a curve of genus two is nontrivial. The idea is to exhibit a Brauer-Manin obstruction to the existence of rational points on a quotient of this principal homogeneous space. In an explicit example we apply the method to show that a specific curve has infinitely many quadratic twists whose Jacobians have nontrivial Tate-Shafarevich group.
\end{abstract}

\section{INTRODUCTION}

By Faltings' Theorem every curve of genus 2 defined over a number field $k$ has only finitely many rational points. Several methods have been developed to find all rational points of a given curve $C$, such as the method of Chabauty-Coleman, the Mordell-Weil sieve, and combinations of these with covering techniques. All these methods require that we know the finitely generated abelian group $J(k)$ of rational points on the Jacobian $J$ of $C$, at least up to a subgroup of finite index. The torsion subgroup of $J(k)$ is generally easy to find (see [7], sections 8.1-2) and the problem is therefore to find the rank $r$ of $J(k)$. The rank can be read off from the size of the group $J(k) / 2 J(k)$ once the torsion subgroup is known. This group fits into an exact sequence

$$
0 \rightarrow J(k) / 2 J(k) \rightarrow \operatorname{Sel}^{(2)}(J / k) \rightarrow \amalg(J / k)[2] \rightarrow 0,
$$

where $\operatorname{Sel}^{(2)}(J / k)$ is the 2-Selmer group of $J / k$ and $\amalg(J / k)[2]$ is the 2-torsion subgroup of the Tate-Shafarevich group $\amalg(J / k)$ of $J / k$. The 2-Selmer group is computable (see 20]). It is, however, not even known whether the Tate-Shafarevich group is always finite. Many papers have been devoted to exhibiting nontrivial elements of $\amalg(J / k)$. In this paper we will follow a new method, suggested by Michael Stoll, which leads to the following result, our main theorem.

Theorem 1.1. Let $S$ be the union of $\{5\}$ with the set of primes that split completely in the field

$$
\mathbb{Q}(\sqrt{-1}, \sqrt{2}, \sqrt{5}, \sqrt{-3(1+\sqrt{2})}, \sqrt{6(1+\sqrt{5})}) .
$$

Then for all $n$ that are products of elements of $S$, the 2-part of the Tate-Shafarevich group of the Jacobian of the curve defined by

$$
y^{2}=-6 n\left(x^{2}+1\right)\left(x^{2}-2 x-1\right)\left(x^{2}+x-1\right)
$$

is nontrivial.

Received by the editor June 16, 2007 and, in revised form, November 19, 2007.

2000 Mathematics Subject Classification. Primary 14H40, 11G10, 14J27, 14J28.

(C) 2008 American Mathematical Society Reverts to public domain 28 years from publication 
Our method uses the fact that every element of $\operatorname{Sel}^{(2)}(J / k)$ can be represented by an everywhere locally solvable 2-covering of $J$. A 2-covering of $J$ is a surface $X$ together with a morphism $\pi: X \rightarrow J$, defined over $k$, such that over the algebraic closure $\bar{k}$ there exists an isomorphism $X_{\bar{k}} \cong J_{\bar{k}}$ making the following diagram commutative:

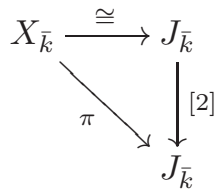

The image of $X$ in $\amalg(J / k)$ is trivial if and only if $X$ has a rational point. Unfortunately, the easiest way to describe $X$ in general is as the intersection of 72 quadrics in $\mathbb{P}^{15}$ (see [7, section 2.3 for the statement). The isomorphism in the diagram above is determined up to translation of $J$ by a 2 -torsion point. Since multiplication by -1 commutes with these translations, it induces a unique involution $\iota$ on $X$. Our strategy to prove that $X$ has no rational points is to show there is a Brauer-Manin obstruction to the existence of rational points on $X / \iota$, or rather on a minimal nonsingular model $V$ of this quotient variety. Note that $V$ is a twist of the Kummer surface associated to $J$.

We will consider curves of genus 2 given in the most general form $y^{2}=f(x)$, where $\operatorname{deg} f=6$. In the case $\operatorname{deg} f=5$, the methods of this paper may be applied after changing coordinates so that there is no rational Weierstrass point at infinity. The methods of [6] can also be used in that case; that article uses a del Pezzo surface which is a quotient of our surface $V$.

The goal of this paper is twofold. In addition to proving the main theorem, we will analyze the geometry of $V$. By [7, Chapter 16, the surface $V$ can be embedded in $\mathbb{P}^{5}$ as the complete intersection of three quadrics. In this reference this is only done when $V$ is a trivial twist, but we will see that it holds for any twist. In this embedding, $V$ contains 32 lines that generically generate the Néron-Severi group of $V$. We will also investigate the intersection pairing among these lines and exhibit 15 pairs of elliptic fibrations, each associated to one of the nontrivial 2-torsion points of $J$. In section 2 we will find the fields of definition of these lines and elliptic fibrations together with explicit equations for them. Then in section [3, we will use the information we have acquired in section 2 to exhibit an explicit example for which we are able to show a Brauer-Manin obstruction. This will be the most important part of the proof of the main theorem.

\section{THE GEOMETRY OF THE SURFACE}

In this section we will investigate the geometry of the K3 surfaces that arise as the quotients of the principal homogeneous spaces under the Jacobian as described in the introduction. These K3 surfaces are twists of the Kummer surface associated to the Jacobian. In [7, sect. 16.2, it is remarked that the Kummer surface itself, i.e., the trivial twist, contains 32 lines. We will give a direct proof that all twists contain 32 lines. We will analyze the Galois action on the set of these lines. We will also describe certain elliptic fibrations, coming in pairs associated to pairs of roots of $f$. In the next section these will be used to find Brauer-Manin obstructions to the existence of rational points on some of these surfaces. In this section we will rarely use the fact that these K3 surfaces are twists of the Kummer surface. Given 
that our goal is to actually implement an algorithm, we will keep everything very explicit, including our proofs. We will, however, refer to [7] at times in order not to lose the context in which our work should be seen.

2.1. The surface. Let $k$ be a field and $W$ a vector space over $k$ of dimension $r \geq 1$. We let $\mathbb{P}(W)$ denote the projective space $(W-\{0\}) / k^{*}$ associated to $W$. The homogeneous coordinate ring of $\mathbb{P}(W)$ is the symmetric algebra $S(\widehat{W})=$ $\bigoplus_{n \geq 0} S^{n}(\widehat{W})$, where $\widehat{W}=\operatorname{Hom}_{k}(W, k)$ is the dual of $W$. Let $\left(x_{1}, \ldots, x_{r}\right)$ be a basis of $\widehat{W}$. This basis yields an isomorphism $\mathbb{P}(W) \rightarrow \mathbb{P}_{k}^{r-1}$ that sends the element $w \in W$ to $\left[x_{1}(w): \ldots: x_{r}(w)\right]$. Thus the $x_{i}$ determine a coordinate system on $\mathbb{P}(W)$. The symmetric algebra $S(\widehat{W})$ is isomorphic to the polynomial ring $k\left[x_{1}, \ldots, x_{r}\right]$.

Let $f \in k[X]$ be a separable polynomial of degree 6 , and set $A_{f}=k[X] / f$. We will also denote the image of $X$ in $A_{f}$ by $X$. Consider $\delta \in A_{f}^{*}$ and set

$$
\mathcal{V}_{f, \delta}=\left\{q \in A_{f}: \exists c_{0}, c_{1}, c_{2} \text { such that } \delta q^{2}=c_{2} X^{2}+c_{1} X+c_{0}\right\} .
$$

Let $V_{f, \delta}$ denote the subset of $\mathbb{P}\left(A_{f}\right)$ corresponding to $\mathcal{V}_{f, \delta}$. We will soon see that $V_{f, \delta}$ is an algebraic set. For any $c \in k^{*}$ we obviously have $A_{c f}=A_{f}, \mathcal{V}_{c f, \delta}=\mathcal{V}_{f, \delta}$, and $V_{c f, \delta}=V_{f, \delta}$. We will often leave any subscript out of the notation that is clear from the context. Let $\left(a_{0}, \ldots, a_{5}\right)$ be the canonical basis of $\widehat{A}$ associated to the basis $\left(1, X, \ldots, X^{5}\right)$ of $A$, so that any $q \in A$ can be written as $q=\sum_{i=0}^{5} a_{i}(q) X^{i}$. As above the $a_{i}$ determine a coordinate system on $\mathbb{P}(A)$. Writing out $\delta q^{2}$, we see that there are quadratic forms $C_{0}, \ldots, C_{5}$ in the homogeneous coordinate ring $S(\widehat{A})$ of $\mathbb{P}(A)$, depending on $f$ and $\delta$, such that $a_{i}\left(\delta q^{2}\right)=C_{i}(q)$ for any $q \in A$. We have $q \in \mathcal{V}$ if and only if we have $C_{i}(q)=0$ for $3 \leq i \leq 5$. This implies that $V$ is an algebraic set in $\mathbb{P}(A)$, defined over $k$ by the three quadrics $C_{3}, C_{4}$, and $C_{5}$. We will express the $C_{i}$ in a new coordinate system, inspired by [7, Chapter 16 .

For any field extension $k^{\prime}$ of $k$ we write $A_{k^{\prime}}=A \otimes_{k} k^{\prime}$, viewed as a vector space over $k^{\prime}$, so that we have $\mathbb{P}\left(A_{k^{\prime}}\right) \cong \mathbb{P}(A)_{k^{\prime}}$. We write $\bar{A}$ and $\bar{V}$ for $A_{\bar{k}}$ and $V_{\bar{k}}$ respectively, where $\bar{k}$ is a fixed algebraic closure $k$. Let $\Omega$ denote the set of roots of $f$ in $\bar{k}$. Then $l=k(\Omega)$ is the splitting field of $f$. For $\omega \in \Omega$ we let $\varphi_{\omega}$ denote the $l$-algebra homomorphism $A_{l} \rightarrow l$ given by $X \mapsto \omega$. The $\varphi_{\omega}$ form a basis of $\widehat{A}_{l}$ and therefore induce a coordinate system on $\mathbb{P}\left(A_{l}\right)$.

Remark 2.1. Let $\left(P_{\omega}\right)_{\omega}$ be the canonical basis of $A_{l}$ associated to the basis $\left(\varphi_{\omega}\right)_{\omega}$ of $\widehat{A}_{l}$. Then for each $q \in A_{l}$ we have

$$
C_{i}(q)=a_{i}\left(\delta q^{2}\right)=a_{i}\left(\sum_{\omega} \varphi_{\omega}\left(\delta q^{2}\right) P_{\omega}\right)=\sum_{\omega} a_{i}\left(P_{\omega}\right) \varphi_{\omega}(\delta) \varphi_{\omega}(q)^{2},
$$

which implies $C_{i}=\sum_{\omega} a_{i}\left(P_{\omega}\right) \delta_{\omega} \varphi_{\omega}^{2}$, with $\delta_{\omega}=\varphi_{\omega}(\delta)$. Note that we have $\varphi_{\omega}=$ $\sum_{i=0}^{5} \omega^{i} a_{i}$, so we can also write the $C_{i}$ in terms of the coordinates $a_{i}$. We can make the constants $a_{i}\left(P_{\omega}\right)$ explicit by setting $P_{\omega}^{\prime}=\prod_{\theta \in \Omega \backslash\{\omega\}}(X-\theta)$. Then $P_{\omega}$ equals the Legendre polynomial $P_{\omega}^{\prime}(\omega)^{-1} P_{\omega}^{\prime}$.

For all $\omega \in \Omega$ we set $\lambda_{\omega}=\varphi_{\omega}\left(P_{\omega}^{\prime}\right)=P_{\omega}^{\prime}(\omega)$ with $P^{\prime}$ as in Remark 2.1 For $j=0,1,2$, set

$$
Q_{j}=\sum_{\omega} \omega^{j} \lambda_{\omega}^{-1} \delta_{\omega} \varphi_{\omega}^{2}
$$


Convention 1. From now on we will assume that the characteristic of $k$ is different from 2 .

Proposition 2.2. The algebraic set $V_{f, \delta}$ is a smooth, geometrically integral complete intersection of the three quadrics $Q_{0}, Q_{1}$, and $Q_{2}$. It is a K3 surface of degree 8.

Proof. Suppose that $f=\sum_{i=0}^{6} f_{i} X^{i}$. The set $V$ is defined by the quadrics $C_{3}, C_{4}$, $C_{5}$, so it is also defined by $Q_{0}^{\prime}=C_{5}, Q_{1}^{\prime}=C_{4}-f_{5} f_{6}^{-1} C_{5}$, and $Q_{2}^{\prime}=C_{3}-f_{5} f_{6}^{-1} C_{4}+$ $\left(f_{5}^{2} f_{6}^{-2}-f_{4} f_{6}^{-1}\right) C_{5}$. From the equations $-f_{5} f_{6}^{-1}=\sum_{\omega} \omega$ and $2 f_{4} f_{6}^{-1}=\sum_{\psi \neq \omega} \psi \omega$ we find that $Q_{i}^{\prime}=Q_{i}$ for $i=0,1,2$. Note that the $3 \times 3$ minors of the Jacobian matrix are equivalent to Vandermonde matrices after scaling the columns. It follows that the quadrics define a smooth complete intersection unless four or more of the scaling factors are 0 , which is equivalent to the vanishing of four or more of the $\varphi_{i}$. The vanishing of the three quadrics would then imply that all of the $\varphi_{i}$ vanish. It follows that $V_{f, \delta}$ is indeed a smooth complete intersection. Every smooth complete intersection of three quadrics in $\mathbb{P}^{5}$ is a K3 surface of degree 8.

Remark 2.3. The statement that $V$ is a K3 surface also follows from the fact that $V$ is the twist by $\delta$ of the desingularized Kummer surface associated to the Jacobian of the curve given by $y^{2}=f$; see [7, Chapter 16 .

Corollary 2.4. The Néron-Severi group $\mathrm{NS}(V)$ of $V$ is free, finitely generated, isomorphic to Pic $V$, and it has a lattice structure induced by the intersection pairing.

Proof. There are injections $\operatorname{Pic} V \hookrightarrow \operatorname{Pic} \bar{V}$ and $\operatorname{Pic}^{0} V \hookrightarrow \operatorname{Pic}^{0} \bar{V}$. As $V$ is a complete intersection by Proposition 2.2. we find from [8], Theorem 1.8, that $\operatorname{Pic}^{0} \bar{V}=0$, so $\mathrm{NS}(V)=\operatorname{Pic} V$. The Néron-Severi group of any projective variety is finitely generated; see [9], ex. V.1.7. Also by [8], Theorem 1.8, we find that Pic $\bar{V}$ is torsion-free, so it is free. In general, the intersection pairing induces a lattice structure on the Néron-Severi group modulo torsion (see [10]), which in this case is isomorphic to Pic $V$.

This theorem also follows from the fact that $V$ is a K3 surface, as shown for characteristic 0 in Prop. VIII.3.2 and on page 120 of [1, and for positive characteristic in Theorem 5 of [2].

Remark 2.5. Consider the net of quadrics $p Q_{0}+q Q_{1}+r Q_{2}$ vanishing on $V$. The curve $C$ in $\mathbb{P}^{2}(p, q, r)$ corresponding to singular quadrics is given by the equation $\operatorname{det}\left(p M_{0}+q M_{1}+r M_{2}\right)=0$ of degree 6 , where $M_{i}$ is the symmetric matrix corresponding to the quadratic form $Q_{i}$. For any $\omega \in \Omega$ the quadric hypersurface corresponding to any point on the line $p+\omega q+\omega^{2} r=0$ is singular at the point in $\mathbb{P}\left(A_{l}\right)$ given by $\varphi_{\theta}=0$ for all $\theta \neq \omega$. This implies that $C$ consists of 6 lines. The 15 intersection points are parametrized by pairs $(\omega, \psi) \in \Omega^{2}$ with $\omega \neq \psi$. The corresponding quadrics are given by $Q_{\omega \psi}=\omega \psi Q_{0}-(\omega+\psi) Q_{1}+Q_{2}$. The hypersurface given by $Q_{\omega \psi}$ is singular at every point on the line $m_{\omega \psi}$ given by $\varphi_{\theta}=0$ for $\theta \neq \omega, \psi$. This hypersurface is a cone over a cone over a quadric $D_{\omega \psi}$ in the $\mathbb{P}^{3}$ obtained by projecting $\mathbb{P}\left(A_{l}\right)$ away from $m_{\omega \psi}$, and therefore contains two families of linear three-spaces. Each family cuts out a family of curves on $V$, given by the two quadrics $Q_{0}, Q_{1}$ in these three-spaces. This yields two elliptic fibrations of $V$, both defined over a quadratic extension of $k(\omega \psi, \omega+\psi)$. We will see later which extension this is. Note that the projection from $m_{\omega \psi}$ induces a 4 -to- 1 map from 
$V$ to $D_{\omega \psi}$. The elliptic fibrations factor through this map. Since $D_{\omega \psi}$ satisfies the Hasse principle this map may be used to obtain information about the arithmetic of $V$.

Let $k^{\prime}$ be any field extension of $k$. For every $z \in A_{k^{\prime}}^{*}$, let $[z]$ denote the automorphism of $\mathbb{P}\left(A_{k^{\prime}}\right)$ induced by multiplication by $z$. Note that $[z]$ maps $V_{\delta}$ isomorphically to $V_{\delta z^{-2}}$, so if $\delta$ is a square in $A_{k^{\prime}}^{*}$, then $V_{\delta}$ is isomorphic to $V_{1}$ over $k^{\prime}$. For any commutative ring $R$ let $\mu(R)$ denote the kernel of the endomorphism $x \mapsto x^{2}$ of $R^{*}$. The scheme Spec $A[t] /\left(t^{2}-1\right)$ represents the functor from the category of $A$-algebras to the category of groups that sends $R$ to $\mu(R)$ in the sense that the elements of $\mu(R)$ are parametrized by the maps from $\operatorname{Spec} R$ to $\operatorname{Spec} A[t] /\left(t^{2}-1\right)$ that respect the map to Spec $A$. Such a map corresponds to the image of $t$ under the associated homomorphism $A[t] /\left(t^{2}-1\right) \rightarrow R$. Let $\mu_{A}$ be the Weil restriction of this scheme from $A$ to $k$. Then $\mu_{A}$ is a $k$-scheme representing the functor that sends a field extension $m$ of $k$ to $\mu\left(A_{m}\right)$. Let $\tilde{\mu}$ be the quotient of $\mu_{A}$ by the automorphism that is induced by $t \mapsto-t$ on Spec $A[t] /\left(t^{2}-1\right)$. Then for all field extensions $m$ of $k$ we have $\tilde{\mu}(m)=\left(\mu\left(A_{\bar{m}}\right) /\langle-1\rangle\right)^{G_{m}}$, where $G_{m}$ is the absolute Galois group of $m$.

Lemma 2.6. The homomorphism $A_{k^{\prime}}^{*} \rightarrow \operatorname{Aut}_{k^{\prime}} \mathbb{P}\left(A_{k^{\prime}}\right)$ that sends $z$ to $[z]$ has kernel $k^{\prime *}$. It induces an injective homomorphism $\tilde{\mu}\left(k^{\prime}\right) \rightarrow \operatorname{Aut}_{k^{\prime}} V_{k^{\prime}}$.

Proof. Note that for $z \in \mu\left(A_{k^{\prime}}\right)$ the automorphisms $[z]$ and $[-z]$ are equal, so the homomorphism $\tilde{\mu}\left(k^{\prime}\right) \rightarrow \operatorname{Aut}_{\bar{k}} V_{\bar{k}}$ is well defined and has image in $\operatorname{Aut}_{k^{\prime}} V_{k^{\prime}}$. We may therefore assume that $k^{\prime}$ is algebraically closed, so that $\tilde{\mu}\left(k^{\prime}\right)=\mu\left(A_{k^{\prime}}\right) /\langle-1\rangle$. Let $\rho$ denote the homomorphism $z \mapsto[z]$ in question. If $\rho(z)$ is the identity, then we have $z \cdot 1=1$ in $\left(A_{k^{\prime}}-\{0\}\right) / k^{\prime *}$, which implies that $z \in k^{\prime *}$. Set $H_{V}=\{\tau \in$ $\operatorname{Aut}_{k^{\prime}} \mathbb{P}\left(A_{k^{\prime}}\right)$ : $\left.\tau(V)=V\right\}$. Since $[z]$ maps $V_{\delta}$ to $V_{\delta z^{-2}}$, the restriction $\rho_{\mu}$ of $\rho$ to $\mu\left(A_{k^{\prime}}\right)$ factors through $H_{V}$. Because $V$ is not contained in a hyperplane, the map $H^{0}\left(\mathbb{P}^{5}, \varnothing_{\mathbb{P}^{5}}(1)\right) \rightarrow H^{0}\left(V, \varnothing_{V}(1)\right)$ is injective. As every element in Aut $_{k^{\prime}} \mathbb{P}_{k^{\prime}}^{5}$ is determined by its action on $H^{0}\left(\mathbb{P}^{5}, \varnothing_{\mathbb{P}^{5}}(1)\right)$, this implies that the restriction map $r: H_{V} \rightarrow$ Aut $_{k^{\prime}} V_{k^{\prime}}$ is injective. Hence, the composition $r \circ \rho_{\mu}: \mu\left(A_{k^{\prime}}\right) \rightarrow$ Aut $_{k^{\prime}} V_{k^{\prime}}$ has kernel ker $\rho_{\mu}=\mu\left(A_{k^{\prime}}\right) \cap k^{\prime *}=\{ \pm 1\}$ and therefore induces the injective homomorphism already mentioned.

For any $\zeta \in \mu\left(A_{k^{\prime}}\right)$ we write $\tilde{\zeta}$ for the image of $\zeta$ in $\tilde{\mu}\left(k^{\prime}\right)$, and $[\zeta]$ or $[\tilde{\zeta}]$ for the induced action by multiplication on $\mathbb{P}\left(A_{k^{\prime}}\right)$ and $V_{k^{\prime}}$. Let $T$ be the Weil restriction of the scheme $\operatorname{Spec} A[t] /\left(t^{2}-\delta\right)$ from $A$ to $k$ and let $\tilde{T}$ be the $k$-scheme that is the quotient of $T$ by the automorphism induced by $t \mapsto-t$ on $\operatorname{Spec} A[t] /\left(t^{2}-\delta\right)$. As for $\mu_{A}$ and $\tilde{\mu}$ above, we can make the identifications

$T(m)=\left\{\xi \in A_{m}: \xi^{2}=\delta\right\} \quad$ and $\quad \tilde{T}(m)=\left(\left\{\xi \in A_{\bar{m}}: \xi^{2}=\delta\right\} /\langle[-1]\rangle\right)^{G_{m}}$,

for every extension $m$ of $k$. Clearly, $T$ is a $k$-torsor under $\mu_{A}$, with the transitive free action of $\mu_{A}\left(k^{\prime}\right)=\mu\left(A_{k^{\prime}}\right)$ on $T\left(k^{\prime}\right)$ given by multiplication in $A_{k^{\prime}}$. Similarly, $\tilde{T}$ is a $k$-torsor under $\tilde{\mu}$.

For any $\xi \in T\left(k^{\prime}\right)$ the 2-dimensional subspace

$$
\mathcal{L}_{\xi}=\left\{\xi^{-1}(s X+t): s, t \in k^{\prime}\right\}
$$

of $A_{k^{\prime}}$ corresponds to a line in $\mathbb{P}\left(A_{k^{\prime}}\right)$, defined over $k^{\prime}$, which is contained in $V_{k^{\prime}}$ and which we will denote by $L_{\xi}$. Since $L_{\xi}=L_{-\xi}$, this implies that to each $\tilde{\xi} \in \tilde{T}\left(k^{\prime}\right)$ we can associate a unique line $L_{\tilde{\xi}}$, namely $L_{\tilde{\xi}}=L_{\xi}$, where $\xi \in T\left(\bar{k}^{\prime}\right)$ is a lift of $\tilde{\xi}$. Let 
$\Lambda\left(k^{\prime}\right)$ denote the set of all lines $L_{\tilde{\xi}}$ corresponding to some $\tilde{\xi} \in \tilde{T}\left(k^{\prime}\right)$. Note that for any $z \in \mu\left(A_{\bar{k}^{\prime}}\right)$ the automorphism $[z]$ maps $L_{\xi}$ to $L_{\xi z^{-1}}$. This induces an action of $\tilde{\mu}\left(k^{\prime}\right)$ on $\Lambda\left(k^{\prime}\right)$.

Lemma 2.7. The action of $\tilde{\mu}\left(k^{\prime}\right)$ on $\Lambda\left(k^{\prime}\right)$ is transitive and free.

Proof. Transitivity follows from the fact that the action of $\mu\left(A_{\overline{k^{\prime}}}\right)$ on $T\left(\overline{k^{\prime}}\right)$ is transitive and that the map $\tilde{T}\left(k^{\prime}\right) \rightarrow \Lambda\left(k^{\prime}\right)$ sending $\tilde{\xi}$ to $L_{\tilde{\xi}}$ is surjective and respects the action of $\mu\left(A_{k^{\prime}}\right)$. To show that the action is free, we may assume that $k^{\prime}$ is algebraically closed. Suppose that for $\tilde{\zeta} \in \tilde{\mu}\left(k^{\prime}\right)$ we have $\tilde{\zeta} L_{\xi}=L_{\xi}$ and let $\zeta \in \mu\left(A_{k^{\prime}}\right)$ be a lift of $\tilde{\zeta}$. Then multiplication by $\zeta$ sends the subspace $\mathcal{L}_{\xi}$ to itself, so it also sends the subspace $W=\left\{s X+t: s, t \in k^{\prime}\right\}$ to itself. In particular, this implies that there are $s, t \in k^{\prime}$ such that $\zeta \cdot 1=s X+t$. From the fact that $\zeta \cdot X \in W$ we then find $s=0$, so $\zeta=t$ is in $\mu\left(A_{k^{\prime}}\right) \cap k^{\prime}=\{ \pm 1\}$, which means $\tilde{\zeta}=1$. We conclude that the action of $\tilde{\mu}\left(k^{\prime}\right)$ on $\Lambda\left(k^{\prime}\right)$ is free.

Lemma 2.8. The map $T\left(\bar{k}^{\prime}\right) \rightarrow \Lambda\left(\bar{k}^{\prime}\right)$ that sends $\xi$ to $L_{\xi}$ induces a bijection $\tilde{T}\left(k^{\prime}\right) \rightarrow \Lambda\left(k^{\prime}\right)$ that respects the action of $\tilde{\mu}\left(k^{\prime}\right)$ and $G(\bar{k} / k)$.

Proof. It is obvious that we obtain a surjective map $\tilde{T}\left(k^{\prime}\right) \rightarrow \Lambda\left(k^{\prime}\right)$ that respects the action of $\tilde{\mu}\left(k^{\prime}\right)$ and $G(\bar{k} / k)$. Injectivity then follows from the fact that $\tilde{\mu}\left(k^{\prime}\right)$ acts transitively and freely on both $\tilde{T}\left(k^{\prime}\right)$ and $\Lambda\left(k^{\prime}\right)$, as we saw in Lemma 2.7

Remark 2.9. Lemmas 2.7 and 2.8 combined say that $\tilde{T}(\bar{k})$ and $\Lambda(\bar{k})$ are isomorphic over $k$ as $k$-torsors under $\tilde{\mu}(\bar{k})$.

Convention 2. For the rest of this section we will suppose that $l$ is contained in $k^{\prime}$.

By the Chinese Remainder Theorem, the map

$$
\varphi_{k^{\prime}}: A_{k^{\prime}} \rightarrow \bigoplus_{\omega \in \Omega} k^{\prime}
$$

induced by the $\varphi_{\omega}$ is an isomorphism, defined over $l$. Note that the induced Galois action on $\bigoplus_{\omega} k^{\prime}$ is given by acting on both the indices and the coefficients in $k^{\prime}$. In other words, for $\sigma \in G(\bar{k} / k)$ we have

$$
\sigma\left(\left(c_{\omega}\right)_{\omega \in \Omega}\right)=\left({ }^{\sigma} c_{\sigma-1}\right)_{\omega \in \Omega} .
$$

It follows that $\mu\left(A_{k^{\prime}}\right)$ and $\tilde{\mu}\left(k^{\prime}\right)$ are isomorphic to $\bigoplus_{\omega}\{ \pm 1\}$ and $\left(\bigoplus_{\omega}\{ \pm 1\}\right) /\{ \pm 1\}$ respectively.

Lemma 2.10. Either the set $\Lambda\left(k^{\prime}\right)$ is empty, or it contains exactly 32 lines.

Proof. Since $\tilde{\mu}\left(k^{\prime}\right)$ has exactly 32 elements, this follows from Lemma 2.7

For $I \subset \Omega$ let $\zeta_{I}$ denote the unique element in $\mu\left(A_{k^{\prime}}\right)$ with $\varphi_{\omega}\left(\zeta_{I}\right)=-1$ for $\omega \in I$ and $\varphi_{\omega}\left(\zeta_{I}\right)=1$ for $\omega \notin I$. Note that we have $\tilde{\zeta}_{I}=\tilde{\zeta}_{\Omega \backslash I}$. We will also denote this element by $\tilde{\zeta}_{\pi}$, where $\pi$ is the partition $\{I, \Omega \backslash I\}$ of $\Omega$. The map from the set $\mathcal{P}(\Omega)$ of all subsets of $\Omega$ to $\mu\left(A_{k^{\prime}}\right)$ that sends $I$ to $\zeta_{I}$ is a bijection. It induces a bijection from the set $\Pi$ of partitions of $\Omega$ into two sets to $\tilde{\mu}\left(k^{\prime}\right)$, sending $\pi$ to $\tilde{\zeta}_{\pi}$. The inverse $\pi: \tilde{\mu}\left(k^{\prime}\right) \rightarrow \Pi$ of the latter bijection is given by

$$
\pi(\tilde{\zeta})=\left\{\left\{\omega: \varphi_{\omega}(\zeta)=1\right\},\left\{\omega: \varphi_{\omega}(\zeta)=-1\right\}\right\},
$$


where $\zeta \in \mu\left(A_{k^{\prime}}\right)$ is a lift of $\tilde{\zeta}$. For any $\pi=\{I, J\} \in \Pi$ the automorphism $\left[\tilde{\zeta}_{\pi}\right]=\left[\zeta_{I}\right]$ is defined over the fixed field of the group $\left\{g \in G(\bar{k} / k):{ }^{g} \pi=\pi\right\}$. Note that $\left[\zeta_{I}\right]$ acts on $\mathbb{P}\left(A_{l}\right)$ by sending the coordinate $\varphi_{\omega}$ to $\pm \varphi_{\omega}$, where the sign is negative if and only if we have $\omega \in I$.

For any $\omega \in \Omega$ and $\xi \in T\left(k^{\prime}\right)$ we let $P_{\xi, \omega}$ denote the point on the line $L_{\xi}$ corresponding to the set

$$
\left\{\xi^{-1} s(X-\omega): s \in k^{\prime}\right\} \subset A_{k^{\prime}} .
$$

For any $z \in \mu\left(A_{k^{\prime}}\right)$ the map $[z]$ sends $P_{\xi, \omega}$ to $P_{\xi z^{-1}, \omega}$. The notation distinguishes the points $P_{\xi, \omega}$, indexed by two subscripts, from the polynomials $P_{\omega}$ from Remark 2.1. which are indexed by only one.

Proposition 2.11. For all $\omega \in \Omega$ and all $\xi \in T\left(k^{\prime}\right)$ we have $\zeta_{\omega} P_{\xi, \omega}=P_{\xi, \omega}$. Two lines $L, L^{\prime} \in \Lambda\left(k^{\prime}\right)$ intersect if and only if there exists an $\omega \in \Omega$ such that $\zeta_{\omega} L=L^{\prime}$, in which case the intersection point is $P_{\xi, \omega}=P_{\xi^{\prime}, \omega}$, where $\xi, \xi^{\prime} \in T\left(k^{\prime}\right)$ are such that $L=L_{\xi}$ and $L^{\prime}=L_{\xi^{\prime}}$.

Proof. One easily checks $\varphi\left(\left(\zeta_{\omega}-1\right)(X-\omega)\right)=0$, so we have $X-\omega=\zeta_{\omega}(X-\omega)$ for all $\omega \in \Omega$. This implies that for all $\xi \in T\left(k^{\prime}\right)$ we have $\zeta_{\omega} P_{\xi, \omega}=P_{\xi \zeta_{\omega}^{-1}, \omega}=P_{\xi, \omega}$, which proves the first statement. Let $\xi, \xi^{\prime} \in T\left(k^{\prime}\right)$ be such that $L=L_{\xi}$ and $L^{\prime}=L_{\xi^{\prime}}$. Suppose there is an $\omega \in \Omega$ such that $\zeta_{\omega} L=L^{\prime}$. Then $L$ and $L^{\prime}$ both go through the point $P_{\xi, \omega}=\zeta_{\omega} P_{\xi, \omega}$, so they intersect.

Conversely, suppose $L$ and $L^{\prime}$ intersect. Then the subspaces $\mathcal{L}_{\xi}$ and $\mathcal{L}_{\xi^{\prime}}$ have a nonzero intersection, so we can choose $s, t, s^{\prime}, t^{\prime} \in k^{\prime}$ such that $\xi^{-1}(s X+t)=$ $\xi^{\prime-1}\left(s^{\prime} X+t^{\prime}\right) \neq 0$. Applying $\varphi_{\omega}$ we find $\varphi_{\omega}(\zeta)(s \omega+t)=s^{\prime} \omega+t^{\prime}$ for all $\omega \in \Omega$, with $\zeta=\xi^{-1} \xi^{\prime} \in \mu\left(A_{k^{\prime}}\right)$. After replacing $\xi^{\prime}$ by $-\xi^{\prime}$ if necessary, we may assume that there are at least three $\omega \in \Omega$ with $\varphi_{\omega}(\zeta)=1$. Then the equation $s x+t=s^{\prime} x+t^{\prime}$ has at least three solutions in $x$, which imply $s^{\prime}=s$ and $t^{\prime}=t$. From $L \neq L^{\prime}$ we deduce $\zeta \neq 1$, so there is an $\omega$ with $\varphi_{\omega}(\zeta) \neq 1$. The equation $\varphi_{\omega}(\zeta)(s \omega+t)=s^{\prime} \omega+t^{\prime}=s \omega+t$ then yields $s \omega+t=0$. Since the equation $s x+t=0$ has at most one solution in $x$, this shows $\zeta=\zeta_{\omega}$ and $\zeta_{\omega} L=L^{\prime}$.

Corollary 2.12. For any line $L \in \Lambda\left(k^{\prime}\right)$ and any elements $\tilde{\zeta}, \tilde{\zeta}^{\prime} \in \tilde{\mu}\left(k^{\prime}\right)$ the lines $\tilde{\zeta} L$ and $\tilde{\zeta}^{\prime} L$ intersect if and only if we have $\tilde{\zeta} \cdot \tilde{\zeta}^{\prime}=\tilde{\zeta}_{\omega}$ for some $\omega \in \Omega$.

Proof. By Lemma 2.7 the element $\tilde{\zeta}^{\prime \prime}=\tilde{\zeta} \cdot \tilde{\zeta}^{\prime}=\tilde{\zeta}^{-1} \cdot \tilde{\zeta}^{\prime}$ is the unique element in $\tilde{\mu}\left(k^{\prime}\right)$ for which we have $\tilde{\zeta}^{\prime \prime} \cdot \tilde{\zeta} L=\tilde{\zeta}^{\prime} L$. By Proposition 2.11 the lines intersect if and only if we have $\tilde{\zeta}^{\prime \prime}=\tilde{\zeta}_{\omega}$ for some $\omega \in \Omega$.

Put $\bar{A}=A_{\bar{k}}, \bar{V}=V_{\bar{k}}$, and $\Lambda=\Lambda(\bar{k})$. For any $L, L^{\prime} \in \Lambda$ we say that $L$ and $L^{\prime}$ have the same or opposite parity if for the unique $\tilde{\zeta} \in \tilde{\mu}(\bar{k})$ with $\tilde{\zeta} L=L^{\prime}$, the number of elements of the sets in the partition $\pi(\tilde{\zeta})$ is even or odd respectively. For any $\omega, \psi \in \Omega$, let $\Phi_{\omega \psi}$ denote the subgroup of $\tilde{\mu}(\bar{k})$ generated by $\tilde{\zeta}_{\omega}$ and $\tilde{\zeta}_{\psi}$.

Lemma 2.13. Let $L, L^{\prime} \in \Lambda$ be different lines of the same parity. Then $L$ and $L^{\prime}$ do not intersect and there are exactly two lines $M$ and $M^{\prime}$ of the opposite parity that intersect both $L$ and $L^{\prime}$. There are $\omega, \psi \in \Omega$ such that the set $\left\{L, L^{\prime}, M, M^{\prime}\right\}$ is an orbit of $\Lambda$ under the action of $\Phi_{\omega \psi}$.

Proof. Let $I \subset \Omega$ be such that $\zeta_{I} L=L^{\prime}$. Then \#I is even, so $L$ and $L^{\prime}$ do not intersect by Corollary 2.12 After replacing $I$ by $\Omega \backslash I$ if necessary, there are $\omega, \psi \in \Omega$, such that $I=\{\omega, \psi\}$. From Corollary 2.12 we deduce that the only 
lines that intersect both $L$ and $L^{\prime}$ are $M=\zeta_{\omega} L$ and $M^{\prime}=\zeta_{\psi} L$. Indeed, the set $\left\{L, L^{\prime}, M, M^{\prime}\right\}$ is an orbit under $\Phi_{\omega \psi}$.

Remark 2.14. Remembering that $\bar{V}$ is a twist of the Kummer surface associated to the Jacobian $J$ of the curve given by $y^{2}=f(x)$, we note that the lines of one parity correspond to the 16 blow-ups of the nodes on the singular surface $J /\langle-1\rangle$. The lines of the other parity correspond to the tropes; see [7, sect. 3.7. The intersection numbers among these lines are well known.

Definition 2.15. A 4-gon is a set $\left\{L, L^{\prime}, M, M^{\prime}\right\}$ of four lines, such that $L$ and $L^{\prime}$ intersect both $M$ and $M^{\prime}$.

By Lemma 2.13 any two lines of the same parity determine a unique 4-gon. All 4-gons arise in this way, because if the lines $L$ and $L^{\prime}$ both intersect a line $M$, then by Lemma 2.13 both $L$ and $L^{\prime}$ are of the opposite parity to $M$, so $L$ and $L^{\prime}$ have the same parity.

Lemma 2.16. Let $\omega, \psi \in \Omega$ and any $I, J \subset \Omega$ be such that the lines $\zeta_{I} L$ and $\zeta_{J} L$ are not in the same orbit under $\Phi_{\omega \psi}$. Then the cardinalities of the sets $I \cap(\Omega \backslash\{\omega, \psi\})$ and $J \cap(\Omega \backslash\{\omega, \psi\})$ have different parity if and only if the line $\zeta_{I} L$ intersects some line in the orbit under $\Phi_{\omega \psi}$ of the line $\zeta_{J} L$ in which case it intersects exactly one line in this orbit.

Proof. Suppose that the sets $I \cap(\Omega \backslash\{\omega, \psi\})$ and $J \cap(\Omega \backslash\{\omega, \psi\})$ are not equal and that their cardinalities have the same parity. Let $\pi=\left\{\pi_{1}, \pi_{2}\right\} \in \Pi$ be such that $\tilde{\zeta}_{\pi}=\tilde{\zeta}_{I} \tilde{\zeta}_{J}$. Then $\zeta_{I} L$ and $\zeta_{J} L$ are in the same orbit under $\Phi_{\omega \psi}$ if and only if we have $\pi_{i} \subset\{\omega, \psi\}$ for $i=1$ or $i=2$. We conclude $\pi_{i} \not \subset\{\omega, \psi\}$ for $i=1,2$. From the parity hypothesis at the beginning of the proof, we deduce that $\pi_{i} \cap(\Omega \backslash\{\omega, \psi\}) \neq \emptyset$ has even parity for $i=1$ and $i=2$. It follows that for each $\tilde{\zeta} \in \Phi_{\omega \psi}$ the sets in the partition $\pi\left(\zeta \zeta_{I} \zeta_{J}\right)=\pi\left(\zeta \zeta_{\pi}\right)$ contain exactly 2 elements of $\Omega \backslash\{\omega, \psi\}$, so $\tilde{\zeta}_{I} L$ does not intersect any of the lines $\tilde{\zeta} \tilde{\zeta}_{J} L$ in the orbit of $\tilde{\zeta}_{J} L$ by Corollary 2.12 .

Conversely, suppose that $I \cap(\Omega \backslash\{\omega, \psi\})$ and $J \cap(\Omega \backslash\{\omega, \psi\})$ have different parity. Then we have $\pi=\{K \cup\{\theta\}, \Omega \backslash(\{\theta\} \cup K)\}$ for some $\theta \in \Omega \backslash\{\omega, \psi\}$ and $K \subset\{\omega, \psi\}$. Then by Corollary 2.12 the line $\zeta_{I} L$ intersects exactly one line in the orbit of $\tilde{\zeta}_{J} L$, namely $\tilde{\zeta}_{K} \tilde{\zeta}_{J} L$.

Lemma 2.17. Let $F_{1}$ be a 4-gon. Then there are exactly 12 lines in $\Lambda$ that do not intersect any line in $F_{1}$. The set of these 12 lines can be partitioned into three 4gons $F_{2}, F_{3}, F_{4}$ and no other subset of this set is a 4-gon. The set of the remaining 16 lines can be partitioned into four 4-gons $G_{1}, G_{2}, G_{3}, G_{4}$ in such a way that, for every $i, j \in\{1,2,3,4\}$, each line in $F_{i}$ intersects exactly one of the lines in $G_{j}$ and each line in $G_{i}$ intersects exactly one of the lines in $F_{j}$. For any different $i, j \in\{1,2,3,4\}$, no line in $F_{i}$ intersects a line in $F_{j}$ and no line in $G_{i}$ intersects a line in $G_{j}$. There are $\omega, \psi \in \Omega$ such that the $F_{i}$ and $G_{i}$ are the orbits of $\Lambda$ under the action of $\Phi_{\omega \psi}$. If $L$ is a line in $F_{1}$, then for $i \in\{2,3,4\}$ there are $\theta, \theta^{\prime} \in \Omega \backslash\{\omega, \psi\}$ such that $F_{i}$ is the orbit of $\zeta_{\theta \theta^{\prime}} L$, and for $j \in\{1,2,3,4\}$ there is a $\theta \in \Omega \backslash\{\omega, \psi\}$ such that $G_{j}$ is the orbit of $\zeta_{\theta} L$.

Proof. Let $\omega, \psi \in \Omega$ be such that $F_{1}$ is an orbit under $\Phi_{\omega \psi}$, and let $L$ denote some line in $F_{1}$. For any $\theta, \theta^{\prime} \in \Omega \backslash\{\omega, \psi\}$ the lines $\zeta_{\theta \theta^{\prime}} L$ and $\zeta_{\omega \theta \theta^{\prime}} L$ do not intersect any line in $F_{1}$ by Lemma 2.16. This gives 12 lines and one checks that the only three 
4-gons contained in the set of these 12 lines are of the form

$$
\left\{\zeta_{\theta_{1} \theta_{2}} L, \zeta_{\theta_{3} \theta_{4}} L, \zeta_{\omega \theta_{1} \theta_{2}} L, \zeta_{\omega \theta_{3} \theta_{4}} L\right\}
$$

for some permutation $\left(\theta_{i}\right)_{i}$ of the elements in $\Omega \backslash\{\omega, \psi\}$. From the equalities $\zeta_{\theta_{3} \theta_{4}} L=\zeta_{\omega \psi \theta_{1} \theta_{2}} L$ and $\zeta_{\omega \theta_{3} \theta_{4}} L=\zeta_{\psi \theta_{1} \theta_{2}} L$ we deduce that these 4-gons are also orbits under $\Phi_{\omega \psi}$, each containing an element $\zeta_{\theta \theta^{\prime}} L$ for some $\theta, \theta^{\prime} \in \Omega \backslash\{\omega, \psi\}$. The remaining 16 lines do intersect a line in $F_{1}$ by Lemma 2.16 and the only four 4-gons contained in the set of these 16 lines are of the form

$$
\left\{\zeta_{\theta} L, \zeta_{\omega \theta} L, \zeta_{\psi \theta} L, \zeta_{\omega \psi \theta} L\right\},
$$

for some $\theta \in \Omega \backslash\{\omega, \psi\}$. Clearly these 4-gons are orbits under $\Phi_{\omega \psi}$ as well. The remaining statements follow from Lemma 2.16.

Definition 2.18. An exhibit is a quadruple $\mathcal{S}=\left\{S_{1}, S_{2}, S_{3}, S_{4}\right\}$ of 4-gons such that the $S_{i}$ are pairwise disjoint and no line in $S_{i}$ intersects a line in $S_{j}$ for $i \neq j$. A gallery is an unordered pair $\left\{\mathcal{S}_{1}, \mathcal{S}_{2}\right\}$ of exhibits such that $\bigcup_{S \in \mathcal{S}_{1}} S$ and $\bigcup_{S \in \mathcal{S}_{2}} S$ are disjoint. For any gallery $\left\{\mathcal{S}_{1}, \mathcal{S}_{2}\right\}$ we say that $\mathcal{S}_{2}$ is the complementary exhibit of $\mathcal{S}_{1}$.

Remark 2.19. Lemma 2.17 says that each 4-gon is contained in a unique exhibit, which is contained in a unique gallery. It also implies that the set of galleries is in bijection with the set of 15 pairs of different elements in $\Omega$. Figure 1 displays a gallery and the intersections among all the 32 lines in $\Lambda$. In Figure 1 we use the notation $I$ for $\zeta_{I} L$ for some fixed line $L$. The elements of $\Omega$ are denoted by $\omega, \psi, 1,2,3,4$. The two exhibits are made up by the 4 -gons on the bottom and the left of the figure respectively.

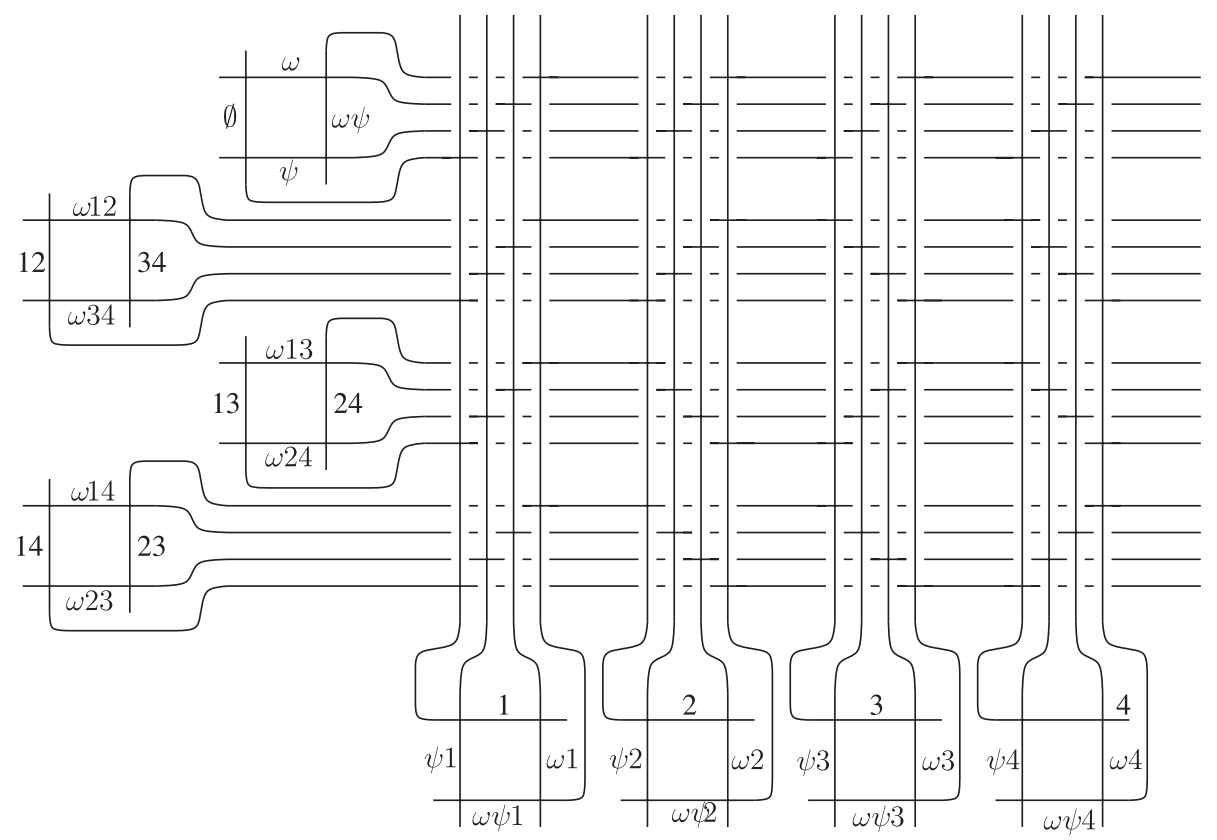

Figure 1. The intersections among the 32 lines in $\Lambda$ 
Lemma 2.20. For each smooth curve $C$ of genus $g$ on a K3 surface, we have $C^{2}=2 g-2$.

Proof. The adjunction formula gives $C \cdot(C+K)=2 g-2$, where $K$ is the canonical divisor of the surface. The lemma now follows from the fact that the canonical divisor of a K3 surface is trivial.

Proposition 2.21. The elements of $\Lambda$ generate a sublattice of the Néron-Severi group $\mathrm{NS}(\bar{V})$ of rank 17 and discriminant 64 .

Proof. Lemma 2.20 implies that $L^{2}=-2$ for all $L \in \Lambda$. From Corollary 2.12 we can deduce all other intersection numbers among elements of $\Lambda$. This gives a $32 \times 32$ Gram matrix of intersection numbers that has rank 17. The matrix also allows us to pick a basis of this sublattice. The Gram matrix with respect to such a basis turns out to have determinant 64 .

Proposition 2.22. The rank of the Néron-Severi group $\mathrm{NS}(\bar{V})$ equals $16+\mathrm{rk} \mathrm{NS}(J)$, where $J$ is the Jacobian of the curve given by $y^{2}=f(x)$.

Proof. The surface $\bar{V}$ is isomorphic to the desingularized Kummer surface associated to $J$ by [7], Chapter 16. The statement therefore follows from [18], Prop. 1.

Proposition 2.23. Generically the lines in $\Lambda$ generate a lattice of finite index in the Néron-Severi group $\mathrm{NS}(\bar{V})$.

Proof. Let $J$ be as in Proposition 2.22. Generically we have $\operatorname{rk} \operatorname{NS}(J)=1$, so $\operatorname{rk} \operatorname{NS}(\bar{V})=17$. By Proposition 2.21 the elements of $\Lambda$ generate a lattice of rank 17 as well, so this lattice has finite index in $\operatorname{NS}(\bar{V})$.

In fact the finite index in Proposition 2.23 is equal to 1 as we will see in Proposition 2.30. The reason for stating that result separately is that one can compute the rank of the Néron-Severi group in explicit cases using methods from 21] and [22].

For any 4-gon $S$ let $D_{S}$ denote the divisor that is the sum of the lines in $S$.

Lemma 2.24. Let $S$ and $S^{\prime}$ be two 4-gons in complementary exhibits. Then the image of $D_{S}+D_{S^{\prime}}$ in Pic $\bar{V}$ is the class of hyperplane sections.

Proof. Let $L$ be a line in $S$ and let $\omega, \psi \in \Omega$ be such that $S$ is the orbit of $L$ under $\Phi_{\omega \psi}$. By Lemma 2.17 the 4-gon $S^{\prime}$ is also an orbit under $\Phi_{\omega \psi}$. It follows from Lemma 2.16 that there is a $\theta \in \Omega \backslash\{\omega, \psi\}$ such that $S^{\prime}$ is the orbit of $\zeta_{\theta} L$. We deduce

$$
D_{S}+D_{S^{\prime}}=L+\zeta_{\omega} L+\zeta_{\psi} L+\zeta_{\psi \omega} L+\zeta_{\theta} L+\zeta_{\theta \omega} L+\zeta_{\theta \psi} L+\zeta_{\theta \psi \omega} L .
$$

One checks that for each $L^{\prime} \in \Lambda$ we have $\left(D_{S}+D_{S^{\prime}}\right) \cdot L^{\prime}=1$. For a hyperplane section $H$ we also have $H \cdot L^{\prime}=1$ for all $L^{\prime} \in \Lambda$. Since the intersection pairing on $\operatorname{Pic} \bar{V}$ is nondegenerate and the lines generically generate a lattice of finite index in Pic $\bar{V}$ by Proposition 2.23, we find that generically $D_{S}+D_{S^{\prime}}$ is a hyperplane section. By specializing, this implies that $D_{S}+D_{S^{\prime}}$ is always a hyperplane section.

Lemma 2.25. Let $S$ and $S^{\prime}$ be two 4-gons in the same exhibit. Then $D_{S}$ and $D_{S^{\prime}}$ are linearly equivalent. 
Proof. Let $S^{\prime \prime}$ be any 4-gon in the complementary exhibit, and let $H$ denote a hyperplane section. Then by Lemma 2.24 both $D_{S}$ and $D_{S^{\prime}}$ are linearly equivalent with $H-D_{S^{\prime \prime}}$.

Proposition 2.26. Let $X$ be a K3 surface over a field and $F$ a reduced and connected curve on $X$ that satisfies $F^{2}=0$. Suppose further that the linear system $|F|$ does not have a base curve. Then there is an elliptic fibration $X \rightarrow \mathbb{P}^{1}$ whose fibers are the elements of $|F|$. Up to an automorphism of $\mathbb{P}^{1}$ this fibration is unique.

Proof. By the adjunction formula we have $F \cdot\left(F+K_{X}\right)=2 p_{a}-2$, where $p_{a}$ is the arithmetic genus of $F$, but $F^{2}=0$ and $K_{X}=0$, so $p_{a}=1$. By the Riemann-Roch theorem for surfaces (9], Thm. V.1.6), we have $h^{0}\left(\varnothing_{X}(-F)\right)-$ $h^{1}\left(\varnothing_{X}(-F)\right)+h^{0}\left(\varnothing_{X}\left(K_{X}+F\right)\right)=1 / 2\left(F \cdot\left(F-K_{X}\right)\right)+1+p_{a}=2$. Now $K_{X}=0$, so $h^{0}\left(\emptyset_{X}\left(K_{X}+F\right)\right)=h^{0}\left(\varnothing_{X}(F)\right)=0$ because $F$ is a nonzero effective divisor. Let us prove that $h^{1}\left(\varnothing_{X}(-F)\right)=0$. From the exact sequence $0 \rightarrow \varnothing_{X}(-F) \rightarrow \emptyset_{X} \rightarrow$ $\varnothing_{F} \rightarrow 0$ of sheaves on $X$ we obtain the exact sequence of cohomology groups

$$
H^{0}\left(X, \varnothing_{X}\right) \rightarrow H^{0}\left(X, \varnothing_{F}\right) \rightarrow H^{1}\left(X, \varnothing_{X}(-F)\right) \rightarrow H^{1}\left(X, \varnothing_{X}\right) .
$$

Since $F$ is projective, reduced, and connected, $H^{0}\left(X, \varnothing_{F}\right)$ consists only of sections constant on $F$, and so the map from $H^{0}\left(X, \varnothing_{X}\right)$ is surjective. On the other hand, $H^{1}\left(X, \varnothing_{X}\right)=0$ because $X$ is a $\mathrm{K} 3$ surface. It follows that $H^{1}\left(X, \varnothing_{X}(-F)\right)=0$ as claimed, and therefore that $h^{0}\left(\varnothing_{X}(-F)\right)=2$.

The only maps whose fibers are elements of the linear system $F$ are those associated to subseries of the complete linear system $|F|$. Since $\varnothing_{X}(-F)$ has a 2dimensional space of sections, the only nonconstant maps of this kind are those associated to the complete linear system. This map is a fibration, for by hypothesis there is no curve contained in all divisors in the linear system $|F|$, so $F^{2}=0$ implies that no two fibers intersect.

Lemma 2.27. For any 4-gon $S$ we have $D_{S}^{2}=0$.

Proof. Write $D_{S}=D_{1}+D_{2}+D_{3}+D_{4}$, where the $D_{i}$ are the geometric irreducible components of $D_{S}$, i.e., the four lines of the 4-gon. By Lemma 2.20 each $D_{i}$ has self-intersection -2 . Also, $D_{i} \cdot D_{i+1}=1$ and $D_{i} \cdot D_{i+2}=0$, with indices read mod 4. The self-intersection of $D_{S}$ is therefore $4 \cdot-2+4 \cdot 2=0$.

Lemma 2.28. Let $\mathcal{S}$ be an exhibit. Then there is an elliptic fibration of $\bar{V}$ for which each 4-gon $S \in \mathcal{S}$ is a fiber. Up to an automorphism of $\mathbb{P}^{1}$ this fibration is unique.

Proof. By Proposition 2.2 the surface $\bar{V}$ is a K3 surface. By Lemma 2.27 we have $D_{S}^{2}=0$ for any 4-gon $S \in \mathcal{S}$. By Lemma 2.25 the effective divisors $D_{S}$ with $S \in \mathcal{S}$ are all contained in the same linear system. The lemma now follows immediately from Proposition 2.26.

Remark 2.29. Since the exhibits come in pairs, so do the elliptic fibrations mentioned in Lemma 2.28. By Remark 2.19 these pairs of fibrations are parametrized by the 15 pairs of elements in $\Omega$.

It is known that generically the lines associated to the nodes and the tropes on the desingularized Kummer surface generate the full Néron-Severi group (cf. Remark 2.14). Together with Propositions 2.21 and 2.23, the following statement is slightly stronger. 
Proposition 2.30. If $\operatorname{rk} \mathrm{NS}(\bar{V})=17$, then the lines in $\Lambda$ generate the full NéronSeveri group $\mathrm{NS}(\bar{V})$.

Proof. Let $L$ denote the sublattice of $\operatorname{NS}(\bar{V})$ generated by the elements of $\Lambda$. By Proposition 2.21 the lattice $L$ has finite index in $\operatorname{NS}(\bar{V})$. Suppose this index is not 1. Then from the equality $64=\operatorname{disc} L=[\operatorname{NS}(\bar{V}): L]^{2} \cdot \operatorname{disc} \operatorname{NS}(\bar{V})$, we find it is divisible by 2 , and disc $\operatorname{NS}(\bar{V})$ is a divisor of $64 / 2^{2}=16$.

Consider the transcendental lattice $T_{\bar{V}}$ of $\bar{V}$, which is the orthogonal complement of $\operatorname{NS}(\bar{V})$ in $H^{2}(\bar{V}, \mathbb{Z})$. As the lattice $H^{2}(\bar{V}, \mathbb{Z})$ is unimodular, we have $\left|\operatorname{disc} T_{\bar{V}}\right|=$ $|\operatorname{disc} \operatorname{NS}(\bar{V})|$, so $\operatorname{disc} T_{\bar{V}}$ is a divisor of 16 as well. However, since $\bar{V}$ is isomorphic to the Kummer surface associated to the Jacobian $J$ of the curve $y^{2}=f(x)$ (see Remark 2.3), we find from [14, Prop. 4.3, that $T_{\bar{V}}$ is isomorphic to $T_{J}(2)$, the transcendental lattice of $J$, scaled by a factor of 2 . Since $T_{\bar{V}}$ has rank $22-17=5$, its discriminant is divisible by $2^{5}=32$. From this contradiction we conclude that the index does equal 1.

2.2. Fields of definition. Recall that $T(F)=\left\{\xi \in A_{F}: \xi^{2}=\delta\right\}$ for any field $F$ for which $\delta$ is contained in $A_{F}$ and that $l=k(\Omega)$ is the splitting field of $f$. Also recall that if $\omega \in F$ is a root of $f$, then $\varphi_{\omega}$ denotes the map $A_{F} \rightarrow F$ that sends $g(X)$ to $g(\omega)$. These maps induce the isomorphism $\varphi: A_{l} \rightarrow \bigoplus_{\omega \in \Omega} l$ given by $X \mapsto\left(\varphi_{\omega}(X)\right)_{\omega}=(\omega)_{\omega}$.

Lemma 2.31. For any $\xi \in T(\bar{k})$ and $\omega, \psi \in \Omega$ we have $\varphi_{\psi}\left(P_{\xi, \omega}\right)=0$ if and only if $\psi=\omega$.

Proof. This follows from the definition of $P_{\xi, \omega}$ and the fact that $\xi \in \bar{A}$ is a unit.

For any object $Y$ to which we can apply every Galois automorphism in $G(\bar{k} / k)$ we will say that $Y$ is defined over the field extension $k^{\prime} \subset \bar{k}$ of $k$ if for all $\sigma \in G\left(\bar{k} / k^{\prime}\right)$ we have ${ }^{\sigma} Y=Y$. The smallest field over which $Y$ is defined will be called the field of definition of $Y$ and denoted by $k(Y)$. Note that every element of $\tilde{T}\left(k^{\prime}\right)$ is defined over $k^{\prime}$, even though it may be represented by an element in $T\left(k^{\prime}\right)$ that is only defined over a quadratic extension of $k^{\prime}$. Note that if $Y=\left(y_{1}, \ldots, y_{n}\right)$ is a sequence, then $k(Y)$ is the composition of all the $k\left(y_{i}\right)$. If $Z=\left\{z_{1}, \ldots, z_{n}\right\}$ is a set, then $k(Z)$ may be strictly smaller then the field of definition of the sequence $\left(z_{1}, \ldots, z_{n}\right)$, a field that we will denote by $k([Z])$.

Lemma 2.32. Inside $\bar{k}$ we have $l \cdot k(L)=k([\Lambda])$ for all $L \in \Lambda$.

Proof. Suppose $\sigma \in G(\bar{k} / k)$ acts trivially on $\Lambda$. Then for all $\omega \in \Omega$ and $\xi \in T(\bar{k})$ the automorphism $\sigma$ fixes the intersection point $P_{\xi, \omega}$ of $L_{\xi}$ and $\zeta_{\omega} L_{\xi}$. The point $P_{\xi, \omega}$ determines $\omega$ uniquely by Lemma 2.31. We conclude that $\sigma$ fixes all $\omega \in \Omega$, so $\sigma$ fixes $l$ and $l$ is contained in $k([\Lambda])$. Clearly, we also have $k(L) \subset k([\Lambda])$, so we find $l \cdot k(L) \subset k([\Lambda])$. For every other $L^{\prime} \in \Lambda$ there is a $\zeta \in \mu\left(A_{l}\right)$ such that $\zeta L=L^{\prime}$. As the automorphism $[\zeta]$ is defined over $l$, we find that $L^{\prime}$ is defined over $l \cdot k(L)$, so $k\left(L^{\prime}\right) \subset l \cdot k(L)$. This holds for all $L^{\prime} \in \Lambda$ so we find $k([\Lambda]) \subset l \cdot k(L)$ and thus $k([\Lambda])=l \cdot k(L)$.

For every $\omega$ we fix a square root $\sqrt{\delta_{\omega}}$ of $\delta_{\omega}=\varphi_{\omega}(\delta)$ in $\bar{k}$, yielding also a fixed square root $\xi_{0}=\varphi^{-1}\left(\left(\sqrt{\delta_{\omega}}\right)_{\omega \in \Omega}\right)$ of $\delta$. Note that with the Legendre polynomials $P_{\omega}$ of Remark 2.1 we can write $\xi_{0}=\sum_{\omega} \sqrt{\delta_{\omega}} P_{\omega}$. We define the fields

$$
m^{\prime}=l\left(\left\{\sqrt{\delta_{\omega}}: \omega \in \Omega\right\}\right) \quad \text { and } \quad m=l\left(\left\{\sqrt{\delta_{\omega}} \sqrt{\delta_{\psi}}: \omega, \psi \in \Omega\right\}\right) .
$$


The square root $\xi_{0}$ of $\delta$ trivializes the torsors $T$ and $\tilde{T}$ under $\mu_{A}$ and $\tilde{\mu}$ respectively, identifying $\zeta \in \mu_{A}(\bar{k})=\mu(\bar{A})$ with $\zeta \xi_{0} \in T(\bar{k})$. By Remark 2.9 the $k$-torsors $\tilde{T}(\bar{k})$ and $\Lambda(\bar{k})$ under $\tilde{\mu}(\bar{k})$ are isomorphic over $k$ as well, identifying the class of $\xi$ in $\tilde{T}(\bar{k})$ with the line $L_{\xi}$. Just after Lemma 2.10 we identified the subset $I \subset \Omega$ with the element $\zeta_{I} \in \mu_{A}(\bar{k})$. Similarly, we write $\xi_{I}=\zeta_{I} \xi_{0}$. We also set $L_{0}=L_{\xi_{0}}$ and write $L_{I}=\zeta_{I} L_{0}=L_{\xi_{I}}$. Note that $L_{I}=L_{\Omega-I}$.

Lemma 2.33. Fix $\sigma \in G(\bar{k} / k)$. Then ${ }^{\sigma} L_{0}=L_{I}$ if and only if $I$ or $\Omega-I$ equals

$$
\left\{\sigma_{\omega}: \omega \in \Omega, \sigma \sqrt{\delta_{\omega}}=\sqrt{\delta_{\sigma_{\omega}}}\right\} .
$$

Proof. This follows immediately from Lemma 2.7 and the equation

$$
\sigma_{\xi_{0}}=\varphi^{-1}\left(\left(\sqrt[\sigma]{\delta_{\sigma^{-1} \omega}}\right)_{\omega \in \Omega}\right)=\zeta_{J} \xi_{0}
$$

where $J$ is the set given in the lemma.

Lemma 2.34. We have $k([\Lambda])=m$.

Proof. For every $\psi \in \Omega$ the element $\sqrt{\delta_{\psi}} \xi_{0}=\sum_{\omega} \sqrt{\delta_{\psi}} \sqrt{\delta_{\omega}} P_{\omega}$ is defined over $m$, where $P_{\omega}$ is the Legendre polynomial introduced in Remark 2.1. The line $L_{0}$ corresponds to the subspace $\left\{\left(\sqrt{\delta_{\psi}} \xi_{0}\right)^{-1}(s X+t): s, t \in m\right\}$ of $A_{m}$, so $L_{0}$ is defined over $m$ as well and we have $k\left(L_{0}\right) \subset m$. From Lemma 2.32 we deduce $k([\Lambda]) \subset m$. For the converse, consider $\sigma \in G(\bar{k} / k([\Lambda]))$. From Lemma 2.33 and the equation $L_{0}={ }^{\sigma} L_{0}$ we find that either we have $\sqrt{\delta_{\omega}}=\sqrt{\delta_{\omega}}$ for all $\omega$, or we have $\sqrt{\delta_{\omega}}=-\sqrt{\delta_{\omega}}$ for all $\omega$. In both cases we find that $\sigma$ acts trivially on $m$, so we also have $m \subset k([\Lambda])$.

Lemma 2.35. Let $\mathcal{S}=\left\{\mathcal{S}_{1}, \mathcal{S}_{2}\right\}$ be a gallery. Then there are $\omega, \psi \in \Omega$ such that we have

$$
k(\mathcal{S})=k(\omega+\psi, \omega \psi) \quad \text { and } \quad k\left(\mathcal{S}_{1}\right)=k\left(\mathcal{S}_{2}\right)=k\left(\omega+\psi, \omega \psi, \prod_{\theta \in \Omega \backslash\{\omega, \psi\}} \sqrt{\delta_{\theta}}\right) .
$$

Proof. Let $\omega, \psi$ be such that the 4 -gons in the $\mathcal{S}_{i}$ are orbits under $\Phi_{\omega \psi}$. Write $k^{\prime}=k(\omega+\psi, \omega \psi)$ and suppose we have $\sigma \in G\left(\bar{k} / k^{\prime}\right)$. Then $\sigma$ fixes the polynomial $(x-\omega)(x-\psi)$, so it permutes $\omega$ and $\psi$. Therefore, $\sigma$ permutes the orbits under $\Phi_{\omega \psi}$, which are the 4-gons in $\mathcal{S}_{1} \cup \mathcal{S}_{2}$ (compare Lemma 2.17). Since every 4-gon is contained in a unique gallery, this implies that $\sigma$ fixes $\mathcal{S}$, so we have $k(\mathcal{S}) \subset k^{\prime}$. For the converse, suppose we have $\sigma \in G(\bar{k} / k(\mathcal{S}))$. Then $\sigma$ permutes the intersection points among any two lines in the same 4-gon in $\mathcal{S}_{1} \cup \mathcal{S}_{2}$. These intersection points are all of the form $P_{\xi, \omega}$ or $P_{\xi, \psi}$ for some $\xi \in T(\bar{k})$. By Lemma 2.31 this implies that $\sigma$ permutes $\omega$ and $\psi$, so it acts trivially on $k^{\prime}$ and we find $k^{\prime} \subset k(\mathcal{S})$, so $k^{\prime}=k(\mathcal{S})$. For the second equality, set $N=\prod_{\theta \in \Omega \backslash\{\omega, \psi\}} \sqrt{\delta_{\theta}}$ and consider $\sigma \in G\left(\bar{k} / k^{\prime}\right)$. Then $\sigma$ fixes $\mathcal{S}$, so it permutes $\mathcal{S}_{1}$ and $\mathcal{S}_{2}$, and $\sigma$ sends $N$ to $\pm N$. Let $n$ denote the number of $\theta \in \Omega \backslash\{\omega, \psi\}$ for which we have $\sqrt{\delta_{\theta}}=-\sqrt{\delta_{\sigma_{\theta}}}$. Then $\sigma$ fixes $N$ if and only if $n$ is even. Let $I \subset \Omega$ be such that ${ }^{\sigma} \xi_{0}=\zeta_{I} \xi_{0}$. Then we have $n=\# I \cap \Omega \backslash\{\omega, \psi\}$ and ${ }^{\sigma} L_{0}=L_{I}$. Suppose that $n=0$ or $n=4$. Then $L_{0}$ and ${ }^{\sigma} L_{0}=L_{I}$ are in the same orbit under $\Phi_{\omega \psi}$, so in the same 4-gon in $\mathcal{S}_{1} \cup \mathcal{S}_{2}$. By Lemma 2.17 each 4-gon is contained in a unique exhibit, so $\sigma$ fixes $\mathcal{S}_{1}$ or $\mathcal{S}_{2}$, and thus both. Now suppose $n \in\{1,2,3\}$. Then ${ }^{\sigma} L_{0}$ is in a different orbit under $\Phi_{\omega \psi}$ from $L_{0}$. By Lemma 2.16 the number $n$ is odd if and only if the line $L_{0}$ intersects some line in the orbit of ${ }^{\sigma} L_{0}$, which, by Lemma 2.17, happens if and only if $L_{0}$ and ${ }^{\sigma} L_{0}$ are in opposite exhibits. 
We conclude that for all $n$ the automorphism $\sigma$ fixes $\mathcal{S}_{1}$ and $\mathcal{S}_{2}$ if and only if $n$ is even, so if and only if $\sigma$ fixes $N$. This implies that $k\left(\mathcal{S}_{1}\right)=k\left(\mathcal{S}_{2}\right)=k^{\prime}(N)$.

Remark 2.36. The first equality of Lemma 2.35 is not surprising as we already saw in Remark 2.19 that galleries are parametrized by pairs of elements in $\Omega$. Note that $k^{\prime}=k(\mathcal{S})=k(\omega+\psi, \omega \psi)$ is the smallest field over which $f$ factors as $f=f_{2} f_{4}$, where $f_{2}$ has degree 2 and roots $\omega$ and $\psi$. It is the field of definition of the 2 -torsion point $(\omega, 0)-(\psi, 0)$ on the Jacobian of the curve $y^{2}=f(x)$. If we set $A_{4}=k^{\prime}[X] / f_{4}$ and we let $\delta^{\prime}$ denote the image of $\delta$ under the natural map $A_{k^{\prime}} \rightarrow A_{4}$, then the element $\prod_{\theta} \sqrt{\delta_{\theta}}$ in Lemma 2.35 is a square root of the norm $N_{A_{4} / k^{\prime}} \delta^{\prime}$ of $\delta^{\prime}$ from $A_{4}$ to $k^{\prime}$. The two elliptic fibrations associated to $\mathcal{S}_{1}$ and $\mathcal{S}_{2}$ in Lemma 2.28 are defined over the field $k\left(\mathcal{S}_{1}\right)=k\left(\mathcal{S}_{2}\right)=k^{\prime}\left(\sqrt{N_{A_{4} / k^{\prime} \delta^{\prime}}}\right)$. We will say that these are the elliptic fibrations associated to the pair $(\omega, \psi)$, or to the factorization $f=f_{2} f_{4}$. The 4-gons in $\mathcal{S}_{1}$ and $\mathcal{S}_{2}$ that make up the fibers of these fibrations are orbits of $\Lambda$ under the group $\Phi_{\omega \psi}$. In section 2.3 we will find explicit equations for these fibrations.

Let $\Lambda_{1}$ and $\Lambda_{2}$ be the two maximal subsets $S$ of $\Lambda$ for which all lines in $S$ have the same parity.

Lemma 2.37. We have $k\left(\Lambda_{1}\right)=k\left(\Lambda_{2}\right)=k(\sqrt{N(\delta)})$, where $N=N_{A / k}$ is the norm from $A$ to $k$.

Proof. Take $\sigma \in G(\bar{k} / k)$. Let $I \subset \Omega$ be such that ${ }^{\sigma} \xi_{0}=\xi_{I}$, and set $n=\# I$. The automorphism $\sigma$ permutes $\Lambda_{1}$ and $\Lambda_{2}$, so it fixes both if and only if $L_{0}$ and ${ }^{\sigma} L_{0}=L_{I}$ have the same parity, i.e., if and only if $n$ is even. Note that $n$ also equals the number of $\omega \in \Omega$ with $\sigma \sqrt{\delta_{\omega}}=-\sqrt{\delta_{\sigma_{\omega}}}$, so $n$ is even if and only if $\sigma$ fixes the element $\prod_{\omega} \sqrt{\delta_{\omega}}=\sqrt{N(\delta)}$. We conclude that $\sigma$ fixes $\Lambda_{1}$ and $\Lambda_{2}$ if and only if $\sigma$ fixes $\sqrt{N(\delta)}$, which shows that $k\left(\Lambda_{1}\right)=k\left(\Lambda_{2}\right)=k(\sqrt{N(\delta)})$.

Let Aut $\Lambda$ denote the group of permutations of $\Lambda$ that respect the intersection pairing. Let $\rho: G(\bar{k} / k) \rightarrow$ Aut $\Lambda$ be the corresponding Galois representation.

Lemma 2.38. The kernel of the representation $\rho: G(\bar{k} / k) \rightarrow$ Aut $\Lambda$ is $G(\bar{k} / m)$.

Proof. This follows from Lemma 2.34.

Proposition 2.39. All extensions among the fields $k \subset l \subset m \subset m^{\prime}$ are Galois and we have exact sequences

$$
\begin{gathered}
1 \rightarrow \operatorname{Gal}\left(m^{\prime} / m\right) \rightarrow \operatorname{Gal}\left(m^{\prime} / l\right) \rightarrow \operatorname{Gal}(m / l) \rightarrow 1 \\
1 \rightarrow \operatorname{Gal}(m / l) \rightarrow \operatorname{Gal}(m / k) \rightarrow \operatorname{Gal}(l / k) \rightarrow 1 \\
1 \rightarrow \operatorname{Gal}\left(m^{\prime} / m\right) \rightarrow \operatorname{Gal}\left(m^{\prime} / l\right) \rightarrow \operatorname{Gal}(m / k) \rightarrow \operatorname{Gal}(l / k) \rightarrow 1 .
\end{gathered}
$$

Proof. The extension $l / k$ is normal because $l$ is the splitting field of $f$ over $k$, and separable because $f$ is. Since $\left[m^{\prime}: l\right]=64=2^{6}$, and we have assumed that char $k \neq 2$, the extension $m^{\prime} / k$ and all subextensions are separable. The group $\operatorname{Gal}(\bar{k} / m)$ is normal in $\operatorname{Gal}(\bar{k} / k)$ because it is the kernel of $\rho$ by Lemma 2.38 This means that $m / k$ is Galois, and therefore so is $m / l$. Similarly, the group $\operatorname{Gal}\left(\bar{k} / m^{\prime}\right)$ is normal in $\operatorname{Gal}(\bar{k} / k)$ because it is the kernel of the representation $\operatorname{Gal}(\bar{k} / k) \rightarrow \operatorname{Aut} T(\bar{k})$. This implies that $m^{\prime} / k$ is Galois, which also follows from the fact that $m^{\prime}$ is obtained from $l$ by adjoining a square root of an element in $l$ as well as the square roots of all conjugates of that element under Gal $(l / k)$. Therefore, 
the extensions $m^{\prime} / m$ and $m^{\prime} / l$ are Galois, too. The first two exact sequences are the standard short exact sequences of Galois groups associated to the double extensions $k \subset l \subset m$ and $l \subset m \subset m^{\prime}$. They can be combined to give the last sequence.

Example 2.40. Let $F$ be any field and define the generic fields

$$
\begin{aligned}
m_{g}^{\prime} & =F\left(\omega_{1}, \ldots, \omega_{6}, d_{0}, \ldots, d_{5}\right)\left[T_{1}, \ldots, T_{6}\right] /\left(T_{j}^{2}-\sum_{i=0}^{5} d_{i} \omega_{j}^{i}: 1 \leq j \leq 6\right), \\
m_{g} & =F\left(\omega_{1}, \ldots, \omega_{6}, d_{0}, \ldots, d_{5},\left\{\epsilon_{i} \epsilon_{j}: 1 \leq i, j \leq 6\right\}\right), \\
l_{g} & =F\left(\omega_{1}, \ldots, \omega_{6}, d_{0}, \ldots, d_{5}\right), \\
k_{g} & =F\left(s_{1}, \ldots, s_{6}, d_{0}, \ldots, d_{5}\right),
\end{aligned}
$$

where $\omega_{1}, \ldots, \omega_{6}, d_{1}, \ldots, d_{6}$ are independent transcendentals, $s_{j}$ denotes the elementary symmetric polynomial of degree $j$ in the variables $\omega_{1}, \ldots, \omega_{6}$, and $\epsilon_{j}$ is the image of $T_{j}$ in $m_{g}^{\prime}$. We have $k_{g} \subset l_{g} \subset m_{g} \subset m_{g}^{\prime}$. Set

$$
f=\prod_{j=1}^{6}\left(X-\omega_{j}\right)=X^{6}-s_{1} X^{5}+s_{2} X^{4}-s_{3} X^{3}+s_{2} X^{4}-s_{5} X^{5}+s_{6} \in k_{g}[X],
$$

and define $A=k_{g}[X] / f$. Recall that, by abuse of notation, we also write $X$ for the image of $X$ in $A$. Set $\delta=\sum_{i=0}^{5} d_{i} X^{i} \in A$. The evaluation maps $\varphi_{j}: A \rightarrow l_{g}$ sending $X$ to $\omega_{j}$ induce an isomorphism $\varphi: A_{l_{g}} \rightarrow \bigoplus_{j=1}^{6} l_{g}$. We have $\epsilon_{j}^{2}=\delta_{j}$ with $\delta_{j}=\varphi_{j}(\delta)$, so the fields $l_{g}, m_{g}$, and $m_{g}^{\prime}$ depend on $k_{g}, f$ and $\delta$ exactly as the corresponding fields without the subscript $g$ for "generic" did before, abbreviating $\omega_{j}$ to $j$ in any index. We will give explicit equations for the intersection points of the lines in $\Lambda_{g}$ in this generic situation. As in Remark 2.1, let $P_{j}$ denote the Legendre polynomial $P_{j}=\prod_{i \neq j}\left(X-\omega_{i}\right) /\left(\omega_{j}-\omega_{i}\right) \in A_{l_{g}}$ for $1 \leq j \leq 6$. Then $\varphi^{-1}$ sends $\left(c_{j}\right)_{j=1}^{6}$ to $\sum_{j=1}^{6} c_{j} P_{j}$. Set $\xi_{0}=\varphi^{-1}\left(\left(\epsilon_{j}\right)_{j}\right)=\sum_{j=1}^{6} \epsilon_{j} P_{j}$. Then we have $\xi_{0}^{2}=\delta$. Let $b_{j i} \in F\left(\omega_{1}, \ldots, \omega_{6}\right)$ be such that $P_{j}=\sum_{i=0}^{5} b_{j i} X^{i}$. In $A_{l_{g}}$ we have $X P_{j}=\omega_{j} P_{j}$, so we find that the $a_{i}$-coordinates of the point $P_{\varepsilon_{0}, \omega_{r}}$ in terms of the $b_{j i}$ are given by the coefficients of

$$
\begin{aligned}
\xi_{0}^{-1}\left(X-\omega_{r}\right) & =\sum_{j=1}^{6} \epsilon_{j}^{-1}\left(X-\omega_{r}\right) P_{j}=\sum_{j=1}^{6} \epsilon_{j}^{-1}\left(\omega_{j}-\omega_{r}\right) P_{j} \\
& =\sum_{i=0}^{5}\left(\sum_{j=1}^{6} \epsilon_{j}^{-1}\left(\omega_{j}-\omega_{r}\right) b_{j i}\right) X^{i} .
\end{aligned}
$$

Multiplying all the coefficients by one of the $\epsilon_{j}^{-1}$ shows that the point $P_{\xi_{0}, \omega_{r}}$ is indeed defined over $m_{g}$. All other intersection points are obtained by replacing some of the $\epsilon_{j}$ by their negatives and $r$ by some $r^{\prime} \in\{1, \ldots, 6\}$. By specialization, these formulas give explicit equations for the intersection points of the lines in $\Lambda$ over any field. This also gives all the lines. Note that the group $\operatorname{Gal}\left(m_{g}^{\prime} / l_{g}\right)$ is isomorphic to $\bigoplus_{j=1}^{6} \mathbb{Z} / 2 \mathbb{Z}$, where the generator of the $j$-th component sends $\epsilon_{j}$ to $-\epsilon_{j}$. The group $\operatorname{Gal}\left(m_{g}^{\prime} / m_{g}\right) \cong \mathbb{Z} / 2 \mathbb{Z}$ embeds diagonally into $\operatorname{Gal}\left(m_{g}^{\prime} / l_{g}\right)$, sending every $\epsilon_{j}$ to $-\epsilon_{j}$. Hence, the group $\Gamma=\operatorname{Gal}\left(m_{g} / l_{g}\right)$ is isomorphic to $(\mathbb{Z} / 2 \mathbb{Z})^{6} /(\mathbb{Z} / 2 \mathbb{Z})$. The group $\operatorname{Gal}\left(l_{g} / k_{g}\right)$ is isomorphic to $S_{6}$. There is a section $\iota^{\prime}$ of the homomorphism $\operatorname{Gal}\left(m_{g}^{\prime} / k_{g}\right) \rightarrow \operatorname{Gal}\left(l_{g} / k_{g}\right)$ that sends an element $\sigma \in \operatorname{Gal}\left(l_{g} / k_{g}\right)$ to the unique lift 
that sends the set $\left\{\epsilon_{1}, \ldots, \epsilon_{6}\right\}$ to itself. The composition $\iota$ of $\iota^{\prime}$ and the restriction map $\operatorname{Gal}\left(m_{g}^{\prime} / k_{g}\right) \rightarrow \operatorname{Gal}\left(m_{g} / k_{g}\right)$ is a section of the homomorphism $\operatorname{Gal}\left(m_{g} / k_{g}\right) \rightarrow$ $\operatorname{Gal}\left(l_{g} / k_{g}\right)$. Through $\iota$ the group $\operatorname{Gal}\left(l_{g} / k_{g}\right) \cong S_{6}$ acts on $\Gamma$ by conjugation. This action is induced by permutation of the components of $\bigoplus_{j=1}^{6} \mathbb{Z} / 2 \mathbb{Z}=\operatorname{Gal}\left(m_{g}^{\prime} / l_{g}\right)$ in the obvious way. Since the middle sequence of Proposition 2.39] splits in this generic case, we find that $\operatorname{Gal}\left(m_{g} / k_{g}\right)$ is isomorphic to the semidirect product $\Gamma \rtimes S_{6}$, which has $32 \cdot 6 !=23040$ elements.

Proposition 2.41. Generically, the representation $\rho: \operatorname{Gal}(\bar{k} / k) \rightarrow$ Aut $\Lambda$ is surjective.

Proof. It suffices to show that $\rho$ is surjective in the case of the generic situation of Example 2.40, so suppose we are in that case. We will use the same notation as in Example 2.40 including abbreviating $\omega_{j}$ to $j$ in indices of lines and points. Take any $\tau \in$ Aut $\Lambda_{g}$ and consider the line $L_{0}=L_{\xi_{0}}$. Since $\Gamma=\operatorname{Gal}\left(m_{g} / l_{q}\right)$ acts transitively on $\Lambda_{g}$, there is a $\sigma_{1} \in \Gamma$ with $\rho\left(\sigma_{1}\right)\left(L_{0}\right)=\tau\left(L_{0}\right)$. Then $\tau^{\prime}=\rho\left(\sigma_{1}\right)^{-1} \tau$ fixes $L_{0}$, so it permutes the six lines $L_{j}$ that intersect $L_{0}$. The corresponding six intersection points are $P_{\xi_{0}, \omega_{j}}$ for $1 \leq j \leq 6$, so $\tau^{\prime}$ induces a unique permutation of the $\omega_{j}$ by Lemma 2.31, which corresponds to an element $\psi \in \operatorname{Gal}\left(l_{g} / k_{g}\right)$. Set $\sigma_{2}=\iota^{\prime}(\psi)$. Then $\sigma_{2}$ sends the set $\left\{\epsilon_{1}, \ldots, \epsilon_{6}\right\}$ to itself, so it fixes $\xi_{0}=\sum_{j=1}^{6} \epsilon_{j} P_{j}$ as both the $\epsilon_{j}$ and the $P_{j}$ are acted on according to their indices. This implies that $\rho\left(\sigma_{2}\right)$ fixes $L_{0}$, while it permutes the intersection points $P_{\xi_{0}, \omega_{j}}$ in the same way $\tau^{\prime}$ does. Therefore $\tau^{\prime \prime}=\rho\left(\sigma_{2}\right)^{-1} \tau^{\prime}$ fixes $L_{0}$ and the six lines $L_{j}$. For $i \neq j$ the line $L_{i j}$ is the unique line that intersects $L_{i}$ and $L_{j}$ that is not equal to $L_{0}$. This implies that $\tau^{\prime \prime}$ also fixes $L_{i j}$. Similarly, the line $L_{i j r}$ is the unique line that intersects both $L_{i j}$ and $L_{i r}$ that is not equal to $L_{i}$. This implies that $\tau^{\prime \prime}$ also fixes $L_{i j r}$. We conclude that $\tau^{\prime \prime}$ is the identity, so $\tau=\rho\left(\sigma_{1} \sigma_{2}\right)$ and $\rho$ is surjective.

By Lemma 2.38 and Proposition 2.41 the generic representation $\rho_{g}: \Gamma \rtimes S_{6} \cong$ $\operatorname{Gal}\left(m_{g} / k_{g}\right) \rightarrow$ Aut $\Lambda_{g} \cong$ Aut $\Lambda$ is an isomorphism. We will denote the composition $\rho_{g} \circ \iota: S_{6} \rightarrow$ Aut $\Lambda$ by $\iota$ as well.

It will be useful to have names for the elements of Aut $\Lambda$. For every set $I \subset \Omega$, let $s_{I} \in$ Aut $\Lambda$ denote the permutation induced by $\left[\zeta_{I}\right]$. Note we have $s_{I}=s_{\Omega \backslash I}$ and $s_{I}$ and $s_{J}$ commute for every $I, J \subset \Omega$. For any permutation $\sigma \in S_{6}=\operatorname{Sym}(\Omega)$, let $t_{\sigma}$ denote the permutation that sends $L_{I}$ to $L_{\sigma_{I}}$. For $\sigma, \tau \in S_{6}$ we have $t_{\sigma} \circ t_{\tau}=t_{\sigma \tau}$, and

$$
t_{\sigma} \circ s_{I}=s_{\sigma_{I}} \circ t_{\sigma} .
$$

Note that the action of $S_{6}$ on $\Lambda$ that we have defined depends on the choice of $L_{0}$, or the $\sqrt{\delta_{\omega}}$, just as the section $\iota$ in Example 2.40 depends on the choice of the square roots $\epsilon_{j}$ of the $\delta_{j}$. We will state some of the following lemmas under an extra condition on $L_{0}$, knowing that the general case may always be obtained by changing some of the $\sqrt{\delta_{\omega}}$ to their negatives and changing the $L_{I}$ and $t_{\sigma}$ accordingly. By specialization of the generic $\omega_{j} \in m_{g}^{\prime}$ of Example 2.40 to the $\omega \in \Omega$, and the $\epsilon_{j}$ to the corresponding $\sqrt{\delta_{\omega}}$, we specialize $k_{g}, l_{g}, m_{g}, \Lambda_{g}$, and the corresponding generic representation $\rho_{g}$ to $k, l, m, \Lambda$, and $\rho$ respectively. Let $r$ denote the associated injective map from $\operatorname{Gal}(m / k)$ to $\operatorname{Gal}\left(m_{g} / k_{g}\right)$. Then we have the following commutative 
diagram:

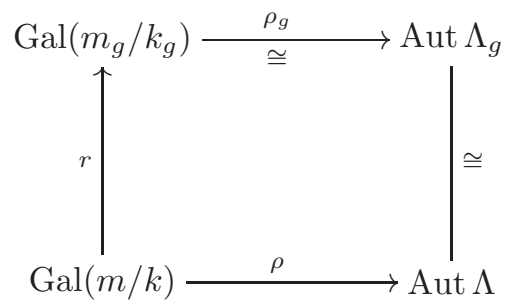

Lemma 2.42. Let $H$ be a subgroup of Aut $\Lambda$ and let $H_{g}$ be the corresponding subgroup of Aut $\Lambda_{g}$. Then the fixed field of $\rho^{-1} H$ is exactly the specialization of the fixed field of $\rho_{g}^{-1} H_{g}$.

Proof. Set $H^{\prime}=\rho^{-1} H$ and $H_{g}^{\prime}=\rho_{g}^{-1} H_{g}$. By the commutative diagram above we have $r^{-1}\left(H_{g}^{\prime}\right)=H^{\prime}$. For every specialization $k^{\prime}$ of a subextension $k_{g}^{\prime}$ of $m_{g}$ over $k_{g}$, we have $\operatorname{Gal}\left(m / k^{\prime}\right)=r^{-1}\left(\operatorname{Gal}\left(m_{g} / k_{g}^{\prime}\right)\right)$. In other words, the fixed field of $H^{\prime}=r^{-1}\left(H_{g}^{\prime}\right)$ is exactly the specialization of the fixed field of $H_{g}^{\prime}$.

For any $\omega, \psi \in \Omega$, let $\Psi_{\omega \psi}$ denote the group generated by $s_{\omega}$ and $s_{\psi}$. Then $\Phi_{\omega \psi}$ acts on $\Lambda$ through $\Psi_{\omega \psi}$. For any exhibit $\mathcal{S}$, let $G_{\mathcal{S}}$ denote the maximal subgroup of Aut $\Lambda$ that fixes $\mathcal{S}$ and let $G_{[\mathcal{S}]}$ denote the maximal subgroup that fixes all 4-gons in $\mathcal{S}$. We will use Lemma 2.42 to find generators of $k([\mathcal{S}])$, the compositum of the fields $k(S)$ for all $S$ in some exhibit $\mathcal{S}$. This field will be used in Section 2.3 to find explicit equations for the elliptic fibration associated to $\mathcal{S}$ in Lemma 2.28

Lemma 2.43. For any exhibit $\mathcal{S}$ the group $G_{\mathcal{S}}$ has order 768 and the natural homomorphism from $G_{\mathcal{S}}$ to the group $\operatorname{Sym}(\mathcal{S})$ of permutations of the 4-gons in $\mathcal{S}$ is surjective. Its kernel is $G_{[\mathcal{S}]}$.

Proof. In the generic case of Example 2.40 , the field $k_{g}(\mathcal{S})$ has degree 30 by Lemma 2.35. Therefore the group $\operatorname{Gal}\left(m_{g} / k_{g}(\mathcal{S})\right)$ has order $23040 / 30=768$. By Lemma 2.38 and Proposition 2.41, the representation $\rho_{g}: \operatorname{Gal}\left(m_{g} / k_{g}\right) \rightarrow$ Aut $\Lambda_{g}$ is an isomorphism, so we find that $G_{\mathcal{S}}$ has order 768 as well. Clearly, the kernel of the homomorphism $\chi: G_{\mathcal{S}} \rightarrow \operatorname{Sym}(\mathcal{S})$ equals $G_{[\mathcal{S}]}$. Let $\omega$ and $\psi$ be such that the 4-gons in $\mathcal{S}$ are orbits under $\Phi_{\omega \psi}$, and set $H=\left\{t_{\sigma}: \sigma \in \operatorname{Sym}(\Omega \backslash\{\omega, \psi\})\right\} \subset$ Aut $\Lambda$. Each $h \in H$ sends orbits under the group $\Phi_{\omega \psi}$ to orbits under the same group, and as we have $h\left(L_{0}\right)=L_{0}$, the permutation $h$ fixes at least one of these orbits, so it fixes the two complementary exhibits associated to the pair $(\omega, \psi)$. We deduce $H \subset G_{\mathcal{S}}$. Without loss of generality, we will assume that $L_{0}$ is not contained in any of the 4-gons of $\mathcal{S}$. Then by Lemma 2.17 for each 4-gon $S$ in $\mathcal{S}$ there is a $\theta \in \Omega \backslash\{\omega, \psi\}$ such that $S$ is the orbit of $L_{\theta}$. It follows that each permutation of $\mathcal{S}$ is induced by a permutation of $\Omega \backslash\{\omega, \psi\}$, so the restriction of $\chi$ to $H$ is surjective, which implies that $\chi$ is surjective.

Lemma 2.44. Let $\mathcal{S}$ be an exhibit, let $\omega, \psi \in \Omega$ be such that the 4-gons of $\mathcal{S}$ are orbits under $\Phi_{\omega \psi}$, and assume that $L_{0}$ is contained in one of the 4-gons of $\mathcal{S}$. Let $\theta_{1}, \theta_{2}, \theta_{3}, \theta_{4}$ be the elements of $\Omega \backslash\{\omega, \psi\}$. Then $G_{[\mathcal{S}]}$ is generated by $s_{\omega}, s_{\psi}$, and $t_{\sigma}$ for $\sigma \in\left\langle(\omega \psi),\left(\theta_{1} \theta_{2}\right)\left(\theta_{3} \theta_{4}\right),\left(\theta_{1} \theta_{3}\right)\left(\theta_{2} \theta_{4}\right)\right\rangle$.

Proof. Set $B=\left\langle\left(\theta_{1} \theta_{2}\right)\left(\theta_{3} \theta_{4}\right),\left(\theta_{1} \theta_{3}\right)\left(\theta_{2} \theta_{4}\right)\right\rangle$ and let $H$ denote the subgroup of Aut $\Lambda$ generated by $s_{\omega}, s_{\psi}, t_{(\omega \psi)}$, and $t_{\sigma}$ for $\sigma \in B$. Note that every $\sigma \in B$ fixes $\omega$ 
and $\psi$. By (2.3) this implies that for every $h \in H$ we have $h \Psi_{\omega \psi} h^{-1}=\Psi_{\omega \psi}$, so $h$ sends orbits under $\Psi_{\omega \psi}$ to orbits under $\Psi_{\omega \psi}$, i.e., $h$ permutes the 4 -gons in $\mathcal{S}$ and its complementary exhibit. The elements $s_{\omega}, s_{\psi}$, and $t_{(\omega \psi)}$ fix each of these 4-gons. Let $S \in \mathcal{S}$ be the 4-gon containing $L_{0}$. We have $t_{\sigma}\left(L_{0}\right)=L_{0}$ for all $\sigma \in B$, so $h$ sends $S$ to $S$ for all $h \in H$. Let $S^{\prime} \in \mathcal{S}$ be a different 4-gon. Then by Lemma 2.17 there are $\theta, \theta^{\prime} \in \Omega \backslash\{\omega, \psi\}$ such that $s_{\theta \theta^{\prime}}(S)=S^{\prime}$. For each $\sigma \in B$ we have

$$
t_{\sigma}\left(s_{\theta \theta^{\prime}}\left(L_{0}\right)\right)=s_{\sigma\left(\theta \theta^{\prime}\right)}\left(t_{\sigma}\left(L_{0}\right)\right)=s_{\sigma\left(\theta \theta^{\prime}\right)}\left(L_{0}\right) .
$$

For all $\sigma \in B$ we have $s_{\theta \theta^{\prime}} S=s_{\sigma\left(\theta \theta^{\prime}\right)} S$, so $t_{\sigma}$ also fixes $S^{\prime}$. We conclude $H \subset G_{[\mathcal{S}]}$. By Lemma 2.43 the order of $G_{[\mathcal{S}]}$ equals $768 / 4 !=32=\# H$, so we have $H=$ $G_{[\mathcal{S}]}$.

We can now find explicit generators of the field $k([\mathcal{S}])$ in the generic case.

Lemma 2.45. Consider the generic case of Example 2.40. Let $\left\{\mathcal{S}, \mathcal{S}^{\prime}\right\}$ be a gallery, such that the 4-gons of $\mathcal{S}$ are orbits under $\Phi_{\omega_{5} \omega_{6}}$, and assume that $L_{0}$ is contained in one of the 4-gons of $\mathcal{S}$. Set $N=\epsilon_{1} \epsilon_{2} \epsilon_{3} \epsilon_{4}, \alpha_{1}=\omega_{1} \omega_{4}+\omega_{2} \omega_{3}, \alpha_{2}=\omega_{1} \omega_{3}+\omega_{2} \omega_{4}$, $\alpha_{3}=\omega_{1} \omega_{2}+\omega_{3} \omega_{4}, \beta_{1}=\epsilon_{1} \epsilon_{4}+\epsilon_{2} \epsilon_{3}, \beta_{2}=\epsilon_{1} \epsilon_{3}+\epsilon_{2} \epsilon_{4}$, and $\beta_{3}=\epsilon_{1} \epsilon_{2}+\epsilon_{3} \epsilon_{4}$. Then $k_{g}^{\prime}=k_{g}\left(\omega_{5}+\omega_{6}, \omega_{5} \omega_{6}, \alpha_{1}, \alpha_{2}, \alpha_{3}\right)$ is the unique $S_{3}$-extension of $k_{g}\left(\left\{\mathcal{S}, \mathcal{S}^{\prime}\right\}\right)=$ $k_{g}\left(\omega_{5}+\omega_{6}, \omega_{5} \omega_{6}\right)$ contained in the $S_{4}$-extension $k_{g}\left(\left\{\mathcal{S}, \mathcal{S}^{\prime}\right\}\right)\left(\omega_{1}, \omega_{2}, \omega_{3}, \omega_{4}\right)$. Set $n_{g}=k_{g}^{\prime}(N)$. Then the field $k_{g}([\mathcal{S}])$ equals $n_{g}\left(\beta_{1}, \beta_{2}, \beta_{3}\right)$ and is an $S_{4}$-extension of $k_{g}(\mathcal{S})=k_{g}\left(\left\{\mathcal{S}, \mathcal{S}^{\prime}\right\}\right)(N)$. Its unique $S_{3}$-subextension is $n_{g}$, and the quadratic subextensions of $n_{g}$ are generated by the $\beta_{i}$ :

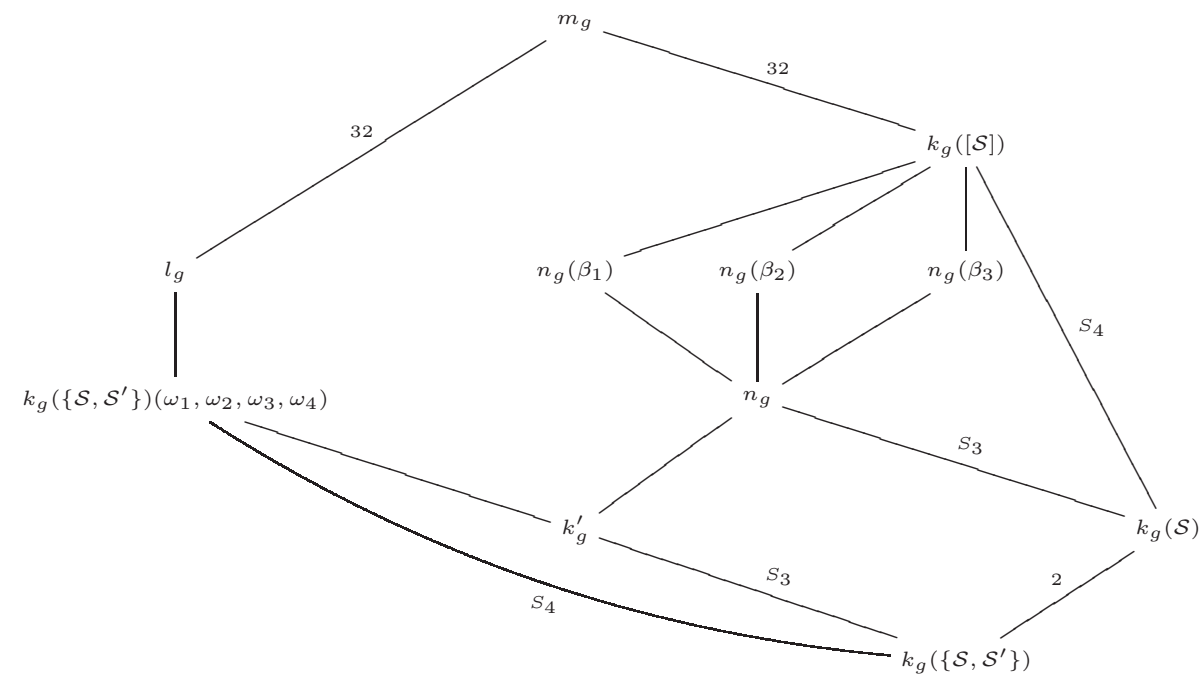

Proof. The first statement is elementary Galois theory. The field $k_{g}([\mathcal{S}])$ is the fixed field of the group $\rho_{g}^{-1}\left(G_{[\mathcal{S}]}\right)$. By Lemma 2.43 this field is an $S_{4}$-extension of the fixed field $k_{g}(\mathcal{S})$ of $G_{\mathcal{S}}$, which equals $k_{g}\left(\omega_{5}+\omega_{6}, \omega_{5} \omega_{6}, N\right)=k_{g}\left(\left\{\mathcal{S}, \mathcal{S}^{\prime}\right\}\right)(N)$ by Lemma 2.35. Using Lemma 2.44 one checks that the group $\rho_{g}^{-1}\left(G_{[\mathcal{S}]}\right)$ acts trivially on $n_{g}\left(\beta_{1}, \beta_{2}, \beta_{3}\right)$, so we conclude $n_{g}\left(\beta_{1}, \beta_{2}, \beta_{3}\right) \subset k_{g}([\mathcal{S}])$. Since $k_{g}^{\prime}$ and $k_{g}(\mathcal{S})$ intersect in $k_{g}\left(\left\{\mathcal{S}, \mathcal{S}^{\prime}\right\}\right)$, we find that the compositum $n_{g}$ is an $S_{3}$-extension of $k_{g}(\mathcal{S})$, and therefore the unique $S_{3}$-extension contained in $k_{g}([\mathcal{S}])$. By elementary Galois theory and group theory this implies that there are three quadratic extensions of $n_{g}$ contained in $k_{g}([\mathcal{S}])$. Note that $\rho_{g}^{-1}\left(s_{\omega_{1} \omega_{4}}\right)$ and $\rho_{g}^{-1}\left(s_{\omega_{1} \omega_{3}}\right)$ act trivially on $n_{g}\left(\beta_{1}\right)$ and $n_{g}\left(\beta_{2}\right)$ respectively, but nontrivially on $\beta_{2}$ and $\beta_{1}$ respectively. We conclude 
that $\beta_{1}$ and $\beta_{2}$ generate two different quadratic extensions of $n_{g}$. By symmetry, $\beta_{3}$ generates a third. This implies $\left[n_{g}\left(\beta_{1}, \beta_{2}, \beta_{3}\right): n_{g}\right] \geq 4=\left[k_{g}([\mathcal{S}]): n_{g}\right]$, so we deduce that $k_{g}([\mathcal{S}])=n_{g}\left(\beta_{1}, \beta_{2}, \beta_{3}\right)$.

The generators of the field $k([\mathcal{S}])$ in any other special case follow immediately.

Corollary 2.46. Let the notation be as in Lemma 2.44. Set $N=\sqrt{\delta_{1} \delta_{2} \delta_{3} \delta_{4}}$, $\alpha_{1}=\theta_{1} \theta_{4}+\theta_{2} \theta_{3}, \alpha_{2}=\theta_{1} \theta_{3}+\theta_{2} \theta_{4}, \alpha_{3}=\theta_{1} \theta_{2}+\theta_{3} \theta_{4}, \beta_{1}=\sqrt{\delta_{1} \delta_{4}}+\sqrt{\delta_{2} \delta_{3}}$, $\beta_{2}=\sqrt{\delta_{1} \delta_{3}}+\sqrt{\delta_{2} \delta_{4}}$, and $\beta_{3}=\sqrt{\delta_{1} \delta_{2}}+\sqrt{\delta_{3} \delta_{4}}$. Then $k_{g}([\mathcal{S}])$ equals

$$
k\left(\omega+\psi, \omega \psi, N, \alpha_{1}, \alpha_{2}, \alpha_{3}, \beta_{1}, \beta_{2}, \beta_{3}\right) .
$$

Proof. Since $k_{g}([\mathcal{S}])$ is the fixed field of the group $\rho^{-1}\left(G_{[\mathcal{S}]}\right)$, it follows from Lemma 2.42 that it suffices to do this in the generic case. This is dealt with in Lemma 2.45 .

2.3. The elliptic fibrations. Let $\mathcal{S}=\left\{S_{1}, S_{2}, S_{3}, S_{4}\right\}$ and $\mathcal{S}^{\prime}=\left\{S_{5}, S_{6}, S_{7}, S_{8}\right\}$ be complementary exhibits. By Lemma 2.28 there is an elliptic fibration $\bar{V} \rightarrow \mathbb{P}^{1}$ such that the 4-gons in $\mathcal{S}$ are some of the fibers. For any $S \in \mathcal{S}$ this fibration can be defined over the field $k(S)$. It is possible, however, that none of the fibers is defined over the field $k(\mathcal{S})$. This implies that the base of the family of fibers is not isomorphic to $\mathbb{P}^{1}$ over $k(\mathcal{S})$. As the base curve does become isomorphic to $\mathbb{P}^{1}$ over some extension field, it is isomorphic to a conic. In this section we will give explicit equations, both for such a conic and for the fibration map in the generic case of Example 2.40. We will use the notation introduced in that example. The equations for any special case follow by specialization. Although the expressions involved become quite large, all computations in this section can still be checked by hand. We recommend, however, to check them with the MAGMA script provided 23]. We will first give the elliptic fibration over the field $k_{g}([\mathcal{S}])$, over which the base curve can be taken to be the projective line.

2.3.1. A fibration over the projective line. After renumbering the elements of $\Omega$, we may assume that the 4-gons of $\mathcal{S}$ are orbits under $\Phi_{\omega_{5} \omega_{6}}$. After applying an automorphism that sends some of the $\epsilon_{i}$ to $-\epsilon_{i}$ (for notation, see Example 2.40), we may also assume that $L_{0}$ is contained in one of the 4 -gons of $\mathcal{S}$. We renumber $S_{1}, \ldots, S_{4}$ and $S_{5}, \ldots, S_{8}$, so that $S_{1}, \ldots, S_{8}$ contain the lines $L_{14}, L_{24}, L_{34}, L_{0}$, $L_{1}, L_{2}, L_{3}$, and $L_{4}$ respectively. In particular, this means

$$
\begin{array}{ll}
S_{1}=\left\{L_{14}, L_{23}, L_{145}, L_{235}\right\}, & S_{5}=\left\{L_{1}, L_{15}, L_{16}, L_{156}\right\}, \\
S_{2}=\left\{L_{13}, L_{24}, L_{135}, L_{245}\right\}, & S_{6}=\left\{L_{2}, L_{25}, L_{26}, L_{256}\right\} .
\end{array}
$$

For notational convenience, we let $N, \alpha_{i}$ and $\beta_{i}$ be as in Lemma 2.45. We also set

$$
\begin{aligned}
& \gamma_{1}=\alpha_{3}-\alpha_{2}, \quad \Delta_{4}=\prod_{1 \leq i<j \leq 4}\left(\omega_{i}-\omega_{j}\right), \\
& \gamma_{2}=\alpha_{1}-\alpha_{3} \text {, } \\
& \gamma_{3}=\alpha_{2}-\alpha_{1}, \quad \kappa_{j}=\prod_{1 \leq i \leq 4}^{i \neq j}\left(\omega_{j}-\omega_{i}\right), \quad 1 \leq j \leq 4, \\
& \eta=\sum_{i=1}^{4} \epsilon_{i} \\
& c_{r}=\text { elementary symmetric polynomial in the } \omega_{j}(1 \leq j \leq 4) \text { of degree } r \text {. }
\end{aligned}
$$

Note that for $1 \leq j \leq 4$ and $J \subset \Omega$ we have $\varphi_{j}\left(\xi_{J}\right)= \pm \epsilon_{j}$, where the sign is negative if and only if we have $j \in J$. For the evaluation of various linear forms at 
the intersection points of the lines in $\Lambda$, it will also be convenient to notice that we have

$$
\sum_{j=1}^{4} \omega_{j}^{r} \kappa_{j}^{-1}= \begin{cases}-c_{4}^{-1} & r=-1 \\ 0 & r=0,1,2 \\ 1 & r=3 .\end{cases}
$$

It will turn out that the elliptic fibration associated to $\mathcal{S}$ factors through the projection of $\mathbb{P}(\bar{A})$ to $\mathbb{P}^{3}$ by the coordinates $\varphi_{i}$ for $1 \leq i \leq 4$, i.e., the projection away from the line given by $\varphi_{i}=0$ for $1 \leq i \leq 4$. The image of $\bar{V}$ under this projection is the nonsingular quadric $D_{\omega_{5} \omega_{6}}$ of Remark 2.5. Note that $\bar{V}$ is contained in the inverse image of $D_{\omega_{5} \omega_{6}}$ under the indicated projection, which is the cone over the cone over $D_{\omega_{5} \omega_{6}}$ in $\mathbb{P}(\bar{A})$, given by $Q=0$ with

$$
Q=\omega_{5} \omega_{6} Q_{0}-\left(\omega_{5}+\omega_{6}\right) Q_{1}+Q_{2}=\sum_{j=1}^{4} \kappa_{j}^{-1} \delta_{j} \varphi_{j}^{2},
$$

as was pointed out in Remark 2.5. Consider the linear forms

$$
\begin{array}{ll}
l_{1}=\sum_{j=1}^{4} \kappa_{j}^{-1} \epsilon_{j} \varphi_{j}, & m_{1}=\sum_{j=1}^{4} \omega_{j}\left(2 \omega_{j}-c_{1}\right) \kappa_{j}^{-1} \epsilon_{j} \varphi_{j}, \\
l_{2}=\sum_{j=1}^{4} \omega_{j} \kappa_{j}^{-1} \epsilon_{j} \varphi_{j}, & m_{2}=\sum_{j=1}^{4}\left(2 c_{4} \omega_{j}^{-1}-c_{3}\right) \kappa_{j}^{-1} \epsilon_{j} \varphi_{j} .
\end{array}
$$

Lemma 2.47. On $\bar{V}$ we have $l_{1} m_{2}=l_{2} m_{1}$. The map $\chi: \bar{V} \rightarrow \mathbb{P}^{1}$ that sends $x$ to $\left[l_{1}(x): m_{1}(x)\right]=\left[l_{2}(x): m_{2}(x)\right]$ is an elliptic fibration, defined over $k_{g}([\mathcal{S}])$. The 4-gons $S_{1}, S_{2}, S_{3}, S_{4}$ are fibers above $\left[-1: \alpha_{1}\right]$, [-1: $\left.\alpha_{2}\right]$, [-1: $\left.\alpha_{3}\right]$, and $[0: 1]$ respectively.

Proof. From (2.4) one easily works out that $m_{1} l_{2}-l_{1} m_{2}=Q$, so on $\bar{V}$ we find $l_{1} m_{2}=l_{2} m_{1}$. The four equations $l_{1}=m_{1}=l_{2}=m_{2}=0$ are linearly independent, so the base locus of the map $\chi$ is given by $\varphi_{i}=0$ on $\bar{V}$, for $1 \leq i \leq 4$. Together with the equations $Q_{0}=Q_{1}=Q_{2}=0$ (see Proposition 2.2), this implies that the base locus of $\chi$ is empty. The fiber $F_{0}$ of $\chi$ above $[a: b]$ is the intersection of $\bar{V}$ with the three-space given by $b l_{i}=a m_{i}$ for $i=1,2$. The quadric $Q$ vanishes on this three-space, in which the fiber $F_{0}$ is therefore given by $Q_{0}=Q_{1}=0$. Since every smooth intersection of two quadrics in $\mathbb{P}^{3}$ is a curve of genus 1 , we deduce that $\chi$ is an elliptic fibration, whose fibers all have degree 4 . The intersection points of the lines in $S_{4}$ are $P_{\xi, \omega_{r}}$ with $\xi \in\left\{\xi_{0}, \xi_{56}\right\}$, and $r \in\{5,6\}$. From (2.4) and the identity

$$
\epsilon_{j} \varphi_{j}\left(\xi_{I}^{-1}\left(X-\omega_{r}\right)\right)= \pm\left(\omega_{j}-\omega_{r}\right),
$$

where the sign is positive if and only if $j \notin I$, we find that the $l_{i}$ vanish on these points, and thus on the lines in $S_{4}$. This implies that $S_{4}$ is contained in the fiber above [0:1]. Since all fibers have degree 4 , the union of the lines in $S_{4}$ is a whole fiber. The lines in $S_{1} \cup S_{2} \cup S_{3}$ do not intersect any line in $S_{4}$, so they are fibral as well, which implies that all $S \in \mathcal{S}$ are fibers of $\chi$. Their images are easily computed by evaluating the $l_{i} / m_{i}$ on the appropriate intersection points $P_{\xi, \omega_{5}}$ of two lines in the 4-gons, using (2.6) and perhaps a computer algebra package to verify that for instance the ratio $l_{1}\left(P_{\xi_{24}, \omega_{5}}\right): m_{1}\left(P_{\xi_{24}, \omega_{5}}\right)$, which is the ratio between

$$
\frac{\omega_{1}-\omega_{5}}{\kappa_{1}}-\frac{\omega_{2}-\omega_{5}}{\kappa_{2}}+\frac{\omega_{3}-\omega_{5}}{\kappa_{3}}-\frac{\omega_{4}-\omega_{5}}{\kappa_{4}}
$$


and

$$
\begin{aligned}
\frac{\omega_{1}\left(2 \omega_{1}-c_{1}\right)\left(\omega_{1}-\omega_{5}\right)}{\kappa_{1}}- & \frac{\omega_{2}\left(2 \omega_{2}-c_{1}\right)\left(\omega_{2}-\omega_{5}\right)}{\kappa_{2}} \\
& +\frac{\omega_{3}\left(2 \omega_{3}-c_{1}\right)\left(\omega_{3}-\omega_{5}\right)}{\kappa_{3}}-\frac{\omega_{4}\left(2 \omega_{4}-c_{1}\right)\left(\omega_{4}-\omega_{5}\right)}{\kappa_{4}},
\end{aligned}
$$

does indeed equal $-1: \alpha_{2}$ (see [23). Since $\chi$ is also given by $\left[\eta l_{i}: \eta m_{i}\right]$, and the polynomials $\eta l_{i}$ and $\eta m_{i}$ are fixed by $\rho_{g}^{-1}\left(G_{[\mathcal{S}]}\right)$, we find that $\chi$ is defined over $k_{g}([\mathcal{S}])$.

Remark 2.48. The map $\chi$ of Lemma 2.47 is in fact defined over $k_{g}\left(S_{1}\right)$. We found the linear forms $l_{i}$ and $m_{i}$ as follows. Using simple linear algebra we found linear forms $h_{1}, h_{2}, h_{3}, h_{4}$ vanishing on the lines in $S_{4} \cup S_{5}, S_{4} \cup S_{6}, S_{3} \cup S_{5}$, and $S_{3} \cup S_{6}$ respectively. The elliptic fibrations given by $\left[h_{1}: h_{2}\right]$ and $\left[h_{3}: h_{4}\right]$ both have the 4-gons in $\mathcal{S}^{\prime}$ as fibers, so they differ by an automorphism of the base, which fixes the points $[0: 1]$ and $[1: 0]$ as both fibrations have the same fibers $S_{5}$ and $S_{6}$ there. This implies that after the appropriate scaling of the $h_{i}$ we may assume $h_{1} h_{4}=h_{2} h_{3}$. The space of linear forms vanishing on $S_{4}$ is spanned by $h_{1}$ and $h_{2}$. We can pick a $k\left(S_{4}\right)$-basis $l_{1}^{\prime}=a h_{1}+b h_{2}$ and $l_{2}^{\prime}=c h_{1}+d h_{2}$ for some $a, b, c, d \in m_{g}$. The fibers $F_{1}$ and $F_{2}$ of the fibration $\left[h_{1}: h_{2}\right]=\left[h_{3}: h_{4}\right]$ above the points $[-b: a]$ and $[-d: c]$ respectively are then defined over $k\left(S_{4}\right)$, as they are the complement of $S_{4}$ inside the hyperplane section given by $l_{1}^{\prime}$ and $l_{2}^{\prime}$ respectively. The space of linear forms vanishing on $F_{1}$ is spanned by $l_{1}^{\prime}$ and $m_{1}^{\prime \prime}=a h_{3}+b h_{4}$. For some $p, q$, the form $m_{1}^{\prime}=p l_{1}^{\prime}+q m_{1}^{\prime \prime}=p l_{1}^{\prime}+a q h_{3}+b q h_{4}$ is also defined over $k\left(S_{4}\right)$. Set $m_{2}^{\prime}=p l_{2}^{\prime}+c q h_{3}+d q h_{4}$. Then from $h_{1} h_{4}=h_{2} h_{3}$ we also find $l_{1}^{\prime} m_{2}^{\prime}=l_{2}^{\prime} m_{1}^{\prime}$ on $\bar{V}$. For some choice of $a, b, c, d, p, q$, the given $l_{i}$ and $m_{i}$ satisfy $l_{i}^{\prime}=\eta l_{i}$, and $m_{i}^{\prime}=\eta m_{i}$.

2.3.2. The fibration over a conic. Let $\tau \in \operatorname{Gal}\left(m_{g} / k_{g}\right)$ denote the automorphism that fixes all the $\omega_{j}$ and the $\epsilon_{j}$ for $j \geq 3$, and sends $\epsilon_{i}$ to $-\epsilon_{i}$ for $i=1,2$. Then $\tau$ induces the nontrivial automorphism of the quadratic extension $k_{g}([\mathcal{S}]) / n_{g}\left(\beta_{3}\right)$, generated by $\beta_{2}$. Since $\tau$ permutes the 4 -gons in $\mathcal{S}$, the elliptic fibration ${ }^{\tau} \chi$ differs from $\chi$ by some automorphism $\psi$ of the base curve $\mathbb{P}^{1}$ by Proposition 2.26, i.e., we have ${ }^{\tau} \chi=\psi \circ \chi$. This implies that the image of the map $\left(\chi,{ }^{\tau} \chi\right): \bar{V} \rightarrow \mathbb{P}^{1} \times \mathbb{P}^{1}$ is contained in the graph of $\psi$. Under the Segre embedding $\mathbb{P}^{1} \times \mathbb{P}^{1} \rightarrow \mathbb{P}^{3}$ this graph maps to a conic that we can embed in $\mathbb{P}^{2}$. We will now make this explicit. For $i=1,2$ we set

$p_{i}=2\left({ }^{\tau} l_{i}\right) l_{i}, \quad q_{i}=\left({ }^{\tau} l_{i}\right) m_{i}+\left({ }^{\tau} m_{i}\right) l_{i}+2 \alpha_{3} l_{i}{ }^{\tau} l_{i}, \quad r_{i}=\beta_{2}^{-1}\left(\left({ }^{\tau} m_{i}\right) l_{i}-\left({ }^{\tau} l_{i}\right) m_{i}\right)$.

Let $C_{1} \subset \mathbb{P}^{2}$ be the conic given by $\gamma_{1} \gamma_{2} p^{2}+q^{2}=\beta_{2}^{2} r^{2}$.

Lemma 2.49. There is an elliptic fibration $\nu_{1}: \bar{V} \rightarrow C_{1}$, defined over $n_{g}\left(\beta_{3}\right)$, given by $x \mapsto\left[p_{i}(x): q_{i}(x): r_{i}(x)\right]$ for $i=1,2$, such that the 4-gons $S_{1}, S_{2}, S_{3}, S_{4}$ are fibers above $\left[2: \gamma_{1}-\gamma_{2}:-\gamma_{3} \beta_{2}^{-1}\right],\left[2: \gamma_{1}-\gamma_{2}: \gamma_{3} \beta_{2}^{-1}\right],\left[0: \beta_{2}: 1\right]$, and $\left[0: \beta_{2}:-1\right]$ respectively.

Proof. Note that the images of the $S_{i}$ under $\chi$, given in Lemma2.47, are $\tau$-invariant. This implies $\left({ }^{\tau} \chi\right)\left(S_{i}\right)={ }^{\tau}\left(\chi\left({ }^{\tau^{-1}} S_{i}\right)\right)=\chi\left({ }^{\tau} S_{i}\right)$. Note also that $\tau$ acts on the $S_{i}$ as the permutation $\left(S_{1} S_{2}\right)\left(S_{3} S_{4}\right)$. By Proposition 2.26 the elliptic fibrations ${ }^{\tau} \chi$ and $\chi$ differ by an automorphism of $\mathbb{P}^{1}$. As an automorphism of $\mathbb{P}^{1}$ is determined by its action on the $\chi\left(S_{i}\right)$, this allows us to check that the automorphism $\psi:[s: t] \mapsto$ $\left[-\alpha_{3} s-t:\left(\alpha_{3}^{2}+\gamma_{1} \gamma_{2}\right) s+\alpha_{3} t\right]$ of $\mathbb{P}^{1}$ satisfies ${ }^{\tau} \chi=\psi \chi$. By Lemma 2.47 it suffices 
to check that $\psi$ switches the points $\left[-1: \alpha_{1}\right]$ and $\left[-1: \alpha_{2}\right]$ and also the points $\left[-1: \alpha_{3}\right]$ and $[0: 1]$ (see [23]). We conclude that $\left(\chi,{ }^{\tau} \chi\right): \bar{V} \rightarrow \mathbb{P}^{1} \times \mathbb{P}^{1}$ is an elliptic fibration over the graph of $\psi$.

Let $h: \mathbb{P}^{1} \times \mathbb{P}^{1} \rightarrow \mathbb{P}^{3}$ denote the modified Segre embedding that sends $([a: b],[c: d])$ to $[x: y: z: w]=\left[a c: a d+b c: \beta_{2}^{-1}(a d-b c): b d\right]$. Then the composition $g=h \circ\left(\chi, \chi^{\tau}\right): \bar{V} \rightarrow \mathbb{P}^{3}$ is $\tau$-invariant, so it is defined over $n_{g}\left(\beta_{3}\right)$. The image of the graph of $\psi$ under $h$ is the conic given by $y^{2}-\beta_{2}^{2} z^{2}=4 x w$ and $\left(\alpha_{3}^{2}+\gamma_{1} \gamma_{2}\right) x+\alpha_{3} y+w=0$. The image of this conic under the projection $\pi: \mathbb{P}^{3} \rightarrow \mathbb{P}^{2},[x, y, z, w] \mapsto\left[2 x: y+2 \alpha_{3} x: z\right]$ is $C_{1}$, so the composition $\nu_{1}=\pi \circ g$ is an elliptic fibration of $\bar{V}$ over $C_{1}$. Since $\pi$ and $g$ are defined over $n_{g}\left(\beta_{3}\right)$, so is $\nu_{1}$. As $\chi$ is given by $\left[l_{i}: m_{i}\right]$, for $i=1,2$, one checks easily that the fibration $\nu_{1}$ is given by $\left[p_{i}: q_{i}: r_{i}\right]$. The images of the fibers are easily computed using the images given in Lemma 2.47 and the fact that we have $\left({ }^{\tau}\left(l_{i} / m_{i}\right)\right)\left(S_{i}\right)=\left(l_{i} / m_{i}\right)\left({ }^{\tau} S_{i}\right)$ as noted above.

We will construct an automorphism $\psi$ of $\mathbb{P}^{2}$ such that $\psi \circ \nu_{1}$ is an elliptic fibration from $\bar{V}$ to a conic $C$, such that both $C$ and the fibration are defined over $k_{g}(\mathcal{S})$, the field of definition of the fibration. We know that there is an elliptic fibration over a conic defined over $k_{g}(\mathcal{S})$ whose fibers include the 4 -gons of $\mathcal{S}$. By Proposition 2.26 it is unique up to an isomorphism of the conic, so we know such a $\psi$ exists. We will do this in two steps by first descending to $n_{g}$ and then to $k_{g}(\mathcal{S})$. Suppose at some step we have a fibration $\nu_{i}: \bar{V} \rightarrow C_{i}$, with $C_{i}$ a conic, defined over a field $K_{i}$ that is Galois over $K_{i+1}$ with Galois group $G_{i}$. We are looking for an automorphism $\psi_{i}$ of $\mathbb{P}^{2}$ such that $\nu_{i+1}=\psi_{i} \circ \nu_{i}: \bar{V} \rightarrow C_{i+1}$ with $C_{i+1}=\psi_{i}\left(C_{i}\right)$ is defined over $K_{i+1}$.

For all $g \in G_{i}$ there is an isomorphism $\sigma(g):{ }^{g} C_{i} \rightarrow C_{i}$ such that $\nu_{i}=\sigma(g) \circ{ }^{g} \nu_{i}$. Since $\sigma(g)^{*}: \operatorname{Pic} C_{i} \rightarrow$ Pic ${ }^{g} C_{i}$ sends the canonical divisor of $C_{i}$ to that of ${ }^{g} C_{i}$, the automorphism $\sigma(g)$ is induced by a unique automorphism of $\mathbb{P}^{2}$, which we will also denote by $\sigma(g)$. These automorphisms satisfy the cocycle condition $\sigma(h g)=$ $\sigma(h) \circ{ }^{h} \sigma(g)$. The automorphism $\psi_{i}$ that we seek satisfies $\psi_{i} \circ \sigma(g)={ }^{g} \psi_{i}$, so $\sigma$ is a coboundary with values in $\operatorname{Aut} \mathbb{P}_{K_{i}}^{2}$ :

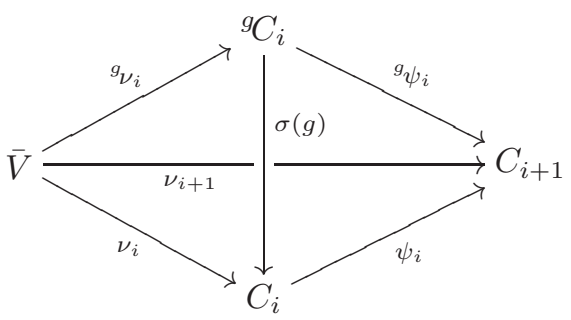

We can find $\psi_{i}$ as follows. Consider the homomorphism $\mathrm{GL}_{3}\left(K_{i}\right) \rightarrow$ Aut $\mathbb{P}_{K_{i}}^{2}$ that maps a matrix $M \in \mathrm{GL}_{3}\left(K_{i}\right)$ to the automorphism that sends $[x: y: z]$ to $\left[x^{\prime}: y^{\prime}: z^{\prime}\right]$ with $\left(x^{\prime} y^{\prime} z^{\prime}\right)^{t}=M(x y z)^{t}$. Through this homomorphism we may identify Aut $\mathbb{P}_{K_{i}}^{2}$ with $\mathrm{PGL}_{3}\left(K_{i}\right)$.

Our first step will be to lift $\sigma$ to a cocycle for $\mathrm{GL}_{3}\left(K_{i}\right)$. The map det: $\mathrm{GL}_{3}\left(K_{i}\right)$ $\rightarrow K_{i}^{*}, M \mapsto \operatorname{det}(M)$ induces a homomorphism $\mathrm{PGL}_{3}\left(K_{i}\right) \rightarrow K_{i}^{*} / K_{i}^{* 3}$. For any $G_{i}$-set $\Xi$, let $Z^{1}(\Xi)$ denote the corresponding set of 1 -cocycles with coefficients in 
$\Xi$. We obtain the following diagram:

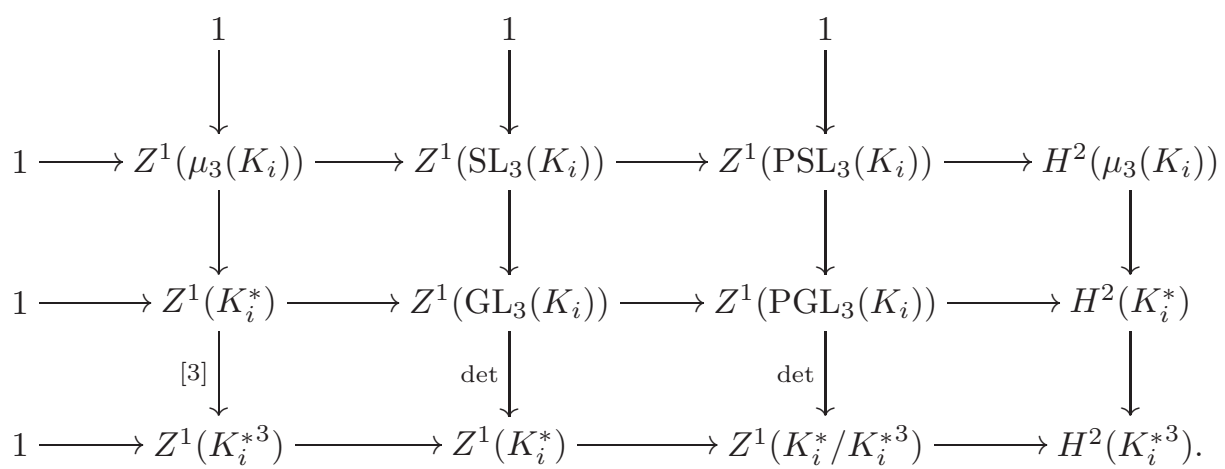

Since $\sigma \in Z^{1}\left(\mathrm{PGL}_{3}\left(K_{i}\right)\right)$ is in fact a coboundary, it maps to zero in $H^{1}\left(\mathrm{PGL}_{3}\left(K_{i}\right)\right)$, so it also maps to zero in $H^{2}\left(K_{i}^{*}\right)$. Therefore we can lift $\sigma$ to an element $\sigma^{\prime} \in$ $Z^{1}\left(\mathrm{GL}_{3}\left(K_{i}\right)\right)$. To do this in practice we note that we are working over a generic field, so we may assume $K_{i}$ has no cube roots of unity. This implies that $\mathrm{SL}_{3}\left(K_{i}\right)$ is isomorphic to the subgroup $\mathrm{PSL}_{3}\left(K_{i}\right)$ of $\mathrm{PGL}_{3}\left(K_{i}\right)$, so an element $M \in \mathrm{GL}_{3}\left(K_{i}\right)$ is uniquely determined by its determinant and its image in $\mathrm{PGL}_{3}\left(K_{i}\right)$. Thus $\sigma^{\prime} \in$ $Z^{1}\left(\mathrm{GL}_{3}\left(K_{i}\right)\right)$ is uniquely determined by its image $\sigma$ and $\operatorname{det} \sigma^{\prime} \in Z^{1}\left(K_{i}^{*}\right)$, which is a lift of $\operatorname{det} \sigma \in Z^{1}\left(K_{i}^{*} / K_{i}^{* 3}\right)$. To find $\sigma^{\prime}$ it therefore suffices to find a lift of $\operatorname{det} \sigma \in Z^{1}\left(K_{i}^{*} / K_{i}^{* 3}\right)$ to $Z^{1}\left(K_{i}^{*}\right)$. In our case $\operatorname{det} \sigma$ will always be trivial, so this step is easy.

The second step is to write $\sigma^{\prime}=Z^{1}\left(\mathrm{GL}_{3}\left(K_{i}\right)\right)$ as a coboundary, which is possible as we have $H^{1}\left(\mathrm{GL}_{3}\left(K_{i}\right)\right)=1$ by a generalization of Hilbert 90 . For this we use a standard trick, discussed in [17, Proposition X.3. For any matrix $M$ we set

$$
b_{M}=\sum_{g \in G} \sigma^{\prime}(g)\left({ }^{g} M\right) \text {. }
$$

One checks that we have $\sigma^{\prime}(h) \cdot{ }^{h} b_{M}=b_{M}$. Choosing $M$ carefully so that $b_{M}$ is invertible, we may take $\psi_{i}$ to be the automorphism associated to the matrix $b_{M}^{-1}$. After some simplifications of these automorphisms we get the following lemma, which can in fact be checked without knowing how we obtained the equations. Let $C_{2} \subset \mathbb{P}^{2}(u, v, w)$ be the conic given by $\gamma_{1} \beta_{1}^{2} u^{2}+\gamma_{2} \beta_{2}^{2} v^{2}+\gamma_{3} \beta_{3}^{2} w^{2}$ and set

$$
\begin{aligned}
u_{i} & =\beta_{1}^{-1}\left(q_{i}+\gamma_{2} p_{i}\right)=\beta_{1}^{-1}\left({ }^{\tau} l_{i} m_{i}+{ }^{\tau} m_{i} l_{i}+2 \alpha_{1} l_{i}{ }^{\tau} l_{i}\right), \\
v_{i} & =\beta_{2}^{-1}\left(q_{i}-\gamma_{1} p_{i}\right)=\beta_{2}^{-1}\left({ }^{\tau} l_{i} m_{i}+{ }^{\tau} m_{i} l_{i}+2 \alpha_{2} l_{i}{ }^{\tau} l_{i}\right), \\
w_{i} & =-\beta_{2} \beta_{3}^{-1} r_{i}=\beta_{3}^{-1}\left({ }^{\tau} l_{i} m_{i}-{ }^{\tau} m_{i} l_{i}\right) .
\end{aligned}
$$

Lemma 2.50. There is an elliptic fibration $\nu_{2}: \bar{V} \rightarrow C_{2}$, defined over $n_{g}$, given by $x \mapsto\left[u_{i}(x): v_{i}(x): w_{i}(x)\right]$ for $i=1,2$, such that the 4-gons $S_{1}, S_{2}, S_{3}, S_{4}$ are fibers above $\left[-\beta_{1}^{-1}: \beta_{2}^{-1}: \beta_{3}^{-1}\right],\left[\beta_{1}^{-1}:-\beta_{2}^{-1}: \beta_{3}^{-1}\right],\left[\beta_{1}^{-1}: \beta_{2}^{-1}:-\beta_{3}^{-1}\right]$, and $\left[\beta_{1}^{-1}: \beta_{2}^{-1}: \beta_{3}^{-1}\right]$ respectively.

Proof. The nontrivial automorphism $\pi$ of the extension $n_{g}\left(\beta_{3}\right) / n_{g}$ is induced by the automorphism that fixes all $\omega_{j}$ and all $\epsilon_{j}$, except for $\epsilon_{1}$ and $\epsilon_{3}$, which are sent to their negatives. It acts on the $S_{i}$ as the permutation $\left(S_{1} S_{3}\right)\left(S_{2} S_{4}\right)$. The composition $\nu_{2}: \bar{V} \rightarrow \mathbb{P}^{2}$ of $\nu_{1}$ and

$$
\psi: \mathbb{P}^{2} \rightarrow \mathbb{P}^{2},[p: q: r] \mapsto\left[\beta_{1}^{-1}\left(q+\gamma_{2} p\right): \beta_{2}^{-1}\left(q-\gamma_{1} p\right):-\beta_{2} \beta_{3}^{-1} r\right]
$$


is given by $x \mapsto\left[u_{i}(x): v_{i}(x): w_{i}(x)\right]$. The inverse of $\psi$ is given by

$$
[u: v: w] \mapsto\left[\beta_{1} u-\beta_{2} v: \gamma_{1} \beta_{1} u+\gamma_{2} \beta_{2} v: \gamma_{3} \beta_{2}^{-1} \beta_{3} w\right] .
$$

Substituting this into the equation for $C_{1}$, we find that the conic $\psi\left(C_{1}\right)$ is equal to $C_{2}$. Alternatively, one checks that we have

$$
\gamma_{1} \beta_{1}^{2} u_{i}^{2}+\gamma_{2} \beta_{2}^{2} v_{i}^{2}+\gamma_{3} \beta_{3}^{2} w_{i}^{2}=4 \gamma_{3} b_{i}{ }^{\tau} l_{i} l_{i} Q
$$

with $b_{1}=\omega_{1}+\omega_{2}-\omega_{3}-\omega_{4}$ and $b_{2}=-c_{4}\left(\omega_{1}^{-1}+\omega_{2}^{-1}-\omega_{3}^{-1}-\omega_{4}^{-1}\right)$ (see 23]). Since $Q$ vanishes on $V$, this also shows that $\nu_{2}$ maps $\bar{V}$ to $C_{2}$. As $\pi$ permutes $\mathcal{S}$, the 4 -gons in $\mathcal{S}$ are also fibers of $\pi_{\nu_{2}}$, so $\nu_{2}$ and ${ }^{\pi} \nu_{2}$ differ by an isomorphism on the base by Proposition 2.26. Therefore, there is a unique isomorphism $h: C_{2} \rightarrow{ }^{\pi} C_{2}=C_{2}$ such that $\pi_{\nu_{2}}=h \circ \nu_{2}$. Since $h$ fixes the anticanonical divisor on $C_{2}$, which determines the embedding of $C_{2}$ in $\mathbb{P}^{2}$, the isomorphism $h$ comes from a unique automorphism of $\mathbb{P}^{2}$, which we will also denote by $h$. Using the points $\nu_{1}\left(S_{i}\right)$ given in Lemma 2.49, one easily computes $\nu_{2}\left(S_{i}\right)=\psi\left(\nu_{1}\left(S_{i}\right)\right)$ to be as claimed. Using the identity $\left({ }^{\pi} \nu_{2}\right)\left(S_{i}\right)=\pi\left(\nu_{2}\left({ }^{-1} S_{i}\right)\right)$ we check that we have $\left({ }^{\pi} \nu_{2}\right)\left(S_{i}\right)=\nu_{2}\left(S_{i}\right)$ for $1 \leq i \leq 4$, so $h$ fixes the four points $\nu_{2}\left(S_{i}\right)$. As these points all lie on $C_{2}$, no three of them are collinear. This implies that the action of $h$ on the four points determines $h$ uniquely, which means that $h$ is the identity, so ${ }^{\pi} \nu_{2}=\nu_{2}$. We conclude that $\nu_{2}$ is defined over the fixed field $n_{g}$ of $\pi$.

Finally, we let $C_{3} \subset \mathbb{P}^{2}(x, y, z)$ be the conic given by

$$
\begin{aligned}
\gamma_{1} \beta_{1}^{2}(x & \left.+\gamma_{1} \Delta_{4} y+\left(\alpha_{2}+\alpha_{3}\right) z\right)^{2}+\gamma_{2} \beta_{2}^{2}\left(x+\gamma_{2} \Delta_{4} y+\left(\alpha_{1}+\alpha_{3}\right) z\right)^{2} \\
& +\gamma_{3} \beta_{3}^{2}\left(x+\gamma_{3} \Delta_{4} y+\left(\alpha_{1}+\alpha_{2}\right) z\right)^{2}=0
\end{aligned}
$$

and we set

$$
\begin{aligned}
& x_{i}=2 \Delta_{4}\left(\left(\alpha_{2} \alpha_{3}-\alpha_{1}^{2}\right) u_{i}+\left(\alpha_{1} \alpha_{3}-\alpha_{2}^{2}\right) v_{i}+\left(\alpha_{1} \alpha_{2}-\alpha_{3}^{2}\right) w_{i}\right), \\
& y_{i}=-\gamma_{1} u_{i}-\gamma_{2} v_{i}-\gamma_{3} w_{i}, \\
& z_{i}=\Delta_{4}\left(\left(\gamma_{2}-\gamma_{3}\right) u_{i}+\left(\gamma_{3}-\gamma_{1}\right) v_{i}+\left(\gamma_{1}-\gamma_{2}\right) w_{i}\right) .
\end{aligned}
$$

Lemma 2.51. There is an elliptic fibration $\nu_{3}: \bar{V} \rightarrow C_{3}$, defined over $k_{g}(\mathcal{S})$, given by $x \mapsto\left[x_{i}(x): y_{i}(x): z_{i}(x)\right]$ for $i=1,2$, such that the 4-gons of $\mathcal{S}$ are fibers of $\nu_{3}$.

Proof. Let $\pi_{1}, \pi_{2} \in \operatorname{Gal}\left(n_{g} / k_{g}(\mathcal{S})\right)$ denote the automorphisms induced by the permutations $\left(\omega_{1} \omega_{2} \omega_{3}\right)$ and $\left(\omega_{1} \omega_{2}\right)$ on the $\omega_{j}$ respectively and the corresponding permutation on the $\epsilon_{j}$. Then $\pi_{1}$ and $\pi_{2}$ induce generators of $\operatorname{Gal}\left(n_{g} / k_{g}(\mathcal{S})\right)$. They act on $\mathcal{S}$, the $\alpha_{j}, \beta_{j}, \gamma_{j}$, and the $\phi_{j}$ as the permutations $(1,2,3)$ and $(1,2)$ on the indices, except that $\pi_{2}$ also negates the $\gamma_{j}$. Note also that we have $\pi_{1}\left(\Delta_{4}\right)=\Delta_{4}$ and $\pi_{2}\left(\Delta_{4}\right)=-\Delta_{4}$. The composition $\nu_{3}: \bar{V} \rightarrow \mathbb{P}^{2}$ of $\nu_{2}$ and $\psi: \mathbb{P}^{2} \rightarrow \mathbb{P}^{2},[u: v:$ $w] \mapsto[x: y: z]$ with

$$
\begin{aligned}
& x=2 \Delta_{4}\left(\left(\alpha_{2} \alpha_{3}-\alpha_{1}^{2}\right) u+\left(\alpha_{1} \alpha_{3}-\alpha_{2}^{2}\right) v+\left(\alpha_{1} \alpha_{2}-\alpha_{3}^{2}\right) w\right), \\
& y=-\gamma_{1} u-\gamma_{2} v-\gamma_{3} w, \\
& z=\Delta_{4}\left(\left(\gamma_{2}-\gamma_{3}\right) u+\left(\gamma_{3}-\gamma_{1}\right) v+\left(\gamma_{1}-\gamma_{2}\right) w\right) .
\end{aligned}
$$

is given by $P \mapsto\left[x_{i}(P): y_{i}(P): z_{i}(P)\right]$. The inverse of $\psi$ is given by $[x: y: z] \mapsto\left[x+\gamma_{1} \Delta_{4} y+\left(\alpha_{2}+\alpha_{3}\right) z: x+\gamma_{2} \Delta_{4} y+\left(\alpha_{1}+\alpha_{3}\right) z: x+\gamma_{3} \Delta_{4} y+\left(\alpha_{1}+\alpha_{2}\right) z\right]$.

Substituting this in the equation for $C_{2}$, we find that the conic $\psi\left(C_{2}\right)$ is equal to $C_{3}$, so $\nu_{3}$ maps $\bar{V}$ to $C_{3}$. Note that for all $g \in \operatorname{Gal}\left(n_{g} / k_{g}(\mathcal{S})\right)$ we have ${ }^{g} C_{3}=C_{3}$. 
As in the proof of Lemma 2.50, for all $g \in \operatorname{Gal}\left(n_{g} / k_{g}(\mathcal{S})\right)$ there is an automorphism $h_{g}$ of $\mathbb{P}^{2}$ such that we have ${ }^{g_{\nu_{3}}}=h_{g} \circ \nu_{3}$. Evaluating $\psi$ at the points $\nu_{2}\left(S_{i}\right)$ given in Lemma 2.50 and using the identity $\left({ }^{g} \nu_{3}\right)\left(S_{i}\right)=g\left(\psi\left(\nu_{2}\left({ }^{-1} S_{i}\right)\right)\right)$, we check that for all $g \in \operatorname{Gal}\left(n_{g} / k_{g}(\mathcal{S})\right)$ we have $\left({ }^{g} \nu_{3}\right)\left(S_{i}\right)=\nu_{3}\left(S_{i}\right)$ for $1 \leq i \leq 4$. It suffices to check this for $g=\pi_{1}, \pi_{2}$. As in the proof of Lemma 2.50 this implies that for all $g \in \operatorname{Gal}\left(n_{g} / k_{g}(\mathcal{S})\right)$ the automorphism $h_{g}$ is the identity, so ${ }^{g} \nu_{3}=\nu_{3}$. We conclude that $\nu_{3}$ is defined over $k_{g}(\mathcal{S})$.

Remark 2.52. Even though the fibration $\nu_{3}$ is defined over $k_{g}(\mathcal{S})$, the polynomials $x_{i}, y_{i}, z_{i}$ that describe $\nu_{3}$ are not, and neither is the defining equation of $C_{3}$. The latter issue is easily resolved by multiplying the given equation for $C_{3}$ by $\Delta_{4}$ to obtain an equation defined over $k(\mathcal{S})$. To settle the former, we can replace $x_{i}, y_{i}, z_{i}$ with Galois-invariant polynomials as follows. Again we descend from the field $k_{g}([\mathcal{S}])$ to $k_{g}(\mathcal{S})$ in steps. The polynomials $l_{i}$ and $m_{i}$ are not defined over $k_{g}([\mathcal{S}])$, but $\eta l_{i}$ and $\eta m_{i}$ are, as they are fixed by the elements of $G_{[\mathcal{S}]}$; see Lemma 2.44, Scaling by $\beta_{2}{ }^{\tau} \eta \eta$, we find that the polynomials $u_{i}^{\prime}=\beta_{2}{ }^{\tau} \eta \eta u_{i}, v_{i}^{\prime}=\beta_{2}{ }^{\tau} \eta \eta v_{i}$, and $w_{i}^{\prime}=\beta_{2}{ }^{\tau} \eta \eta w_{i}$ are also defined over $n_{g}\left(\beta_{3}\right)$ and define $\nu_{2}$ as well. Since $\nu_{2}$ is defined over $n_{g}$, the polynomials ${ }^{\pi} u_{i}^{\prime},{ }^{\pi} v_{i}^{\prime}$, and ${ }^{\pi} w_{i}^{\prime}$, define $\nu_{2}$ as well, where $\pi$ is the nontrivial automorphism of $n_{g}\left(\beta_{3}\right) / n_{g}$ as in the proof of Lemma 2.50. This implies that $\nu_{2}$ can also be defined by $\left[u_{i}^{\prime \prime}: v_{i}^{\prime \prime}: w_{i}^{\prime \prime}\right]$ with $u_{i}^{\prime \prime}=u_{i}^{\prime}+{ }^{\pi} u_{i}^{\prime}, v_{i}^{\prime \prime}=v_{i}^{\prime}+{ }^{\pi} v_{i}^{\prime}$, and $w_{i}^{\prime \prime}=w_{i}^{\prime}+{ }^{\pi} w_{i}^{\prime}$ unless these polynomials vanish on $V$, which in this generic case they do not. One checks, for instance, that we have

$$
\begin{array}{r}
\frac{\beta_{1} u_{1}^{\prime \prime}}{\beta_{2}}=8\left(\epsilon_{1} \epsilon_{2}-\epsilon_{3} \epsilon_{4}\right)\left(\frac{\kappa_{1}^{-1} \delta_{1} \varphi_{1}^{2}+\kappa_{4}^{-1} \delta_{4} \varphi_{4}^{2}}{\omega_{1}-\omega_{4}}+\frac{\kappa_{2}^{-1} \delta_{2} \varphi_{2}^{2}+\kappa_{3}^{-1} \delta_{3} \varphi_{3}^{2}}{\omega_{2}-\omega_{3}}\right) \\
+4\left(\delta_{1}+\delta_{2}-\delta_{3}-\delta_{4}\right)\left(\omega_{1}-\omega_{2}-\omega_{3}+\omega_{4}\right)\left(\left(\omega_{1}-\omega_{2}\right) \kappa_{1}^{-1} \kappa_{2}^{-1} \epsilon_{1} \epsilon_{2} \varphi_{1} \varphi_{2}\right. \\
\left.+\left(\omega_{3}-\omega_{4}\right) \kappa_{3}^{-1} \kappa_{4}^{-1} \epsilon_{3} \epsilon_{4} \varphi_{3} \varphi_{4}\right)
\end{array}
$$

(see [23]). Using the same, trick we descend to $k_{g}(\mathcal{S})$. The polynomials

$$
\begin{aligned}
x_{i}^{\prime} & =2 \Delta_{4}\left(\left(\alpha_{2} \alpha_{3}-\alpha_{1}^{2}\right) u_{i}^{\prime \prime}+\left(\alpha_{1} \alpha_{3}-\alpha_{2}^{2}\right) v_{i}^{\prime \prime}+\left(\alpha_{1} \alpha_{2}-\alpha_{3}^{2}\right) w_{i}^{\prime \prime}\right), \\
y_{i}^{\prime} & =-\gamma_{1} u_{i}^{\prime \prime}-\gamma_{2} v_{i}^{\prime \prime}-\gamma_{3} w_{i}^{\prime \prime}, \\
z_{i}^{\prime} & =\Delta_{4}\left(\left(\gamma_{2}-\gamma_{3}\right) u_{i}^{\prime \prime}+\left(\gamma_{3}-\gamma_{1}\right) v_{i}^{\prime \prime}+\left(\gamma_{1}-\gamma_{2}\right) w_{i}^{\prime \prime}\right),
\end{aligned}
$$

are defined over $n_{g}$ and $\nu_{3}$ is defined by $\left[x_{i}^{\prime}: y_{i}^{\prime}: z_{i}^{\prime}\right]$. Since $\nu_{3}$ is defined over $k_{g}(\mathcal{S})$, it is also defined by $\left[{ }^{g} x_{i}^{\prime}:{ }^{g} y_{i}^{\prime}:{ }^{g} z_{i}^{\prime}\right]$ for any $g \in \operatorname{Gal}\left(n_{g} / k_{g}(\mathcal{S})\right)$ and therefore by $\left[x_{i}^{\prime \prime}: y_{i}^{\prime \prime}: z_{i}^{\prime \prime}\right]$ with $x_{i}^{\prime \prime}=\sum_{g \in \operatorname{Gal}\left(n_{g} / k_{g}(\mathcal{S})\right)}{ }^{g} x_{i}^{\prime}, y_{i}^{\prime \prime}=\sum_{g \in \operatorname{Gal}\left(n_{g} / k_{g}(\mathcal{S})\right)}{ }^{g} y_{i}^{\prime}$, and $z_{i}^{\prime \prime}=\sum_{g \in \operatorname{Gal}\left(n_{g} / k_{g}(\mathcal{S})\right)}{ }^{g} z_{i}^{\prime}$, unless these polynomials, defined over $k(\mathcal{S})$, vanish on $V$. An explicit computation shows that they do not. Over $k_{q}(\mathcal{S})$ we can write $f=f_{2} f_{4}$ with $f_{4}=X^{4}-c_{1} X^{3}+c_{2} X^{2}-c_{3} X+c_{4}$, where the $c_{r}$ are the symmetric polynomials in the $\omega_{j}(1 \leq j \leq 4)$ of degree $r$. The image in $A_{4}=k_{g}[X] / f_{4}$ of $\delta \in A$ can be written as $\delta^{\prime}=d_{3} X^{3}+d_{2} X^{2}+d_{1} X+d_{0}$ with $d_{i} \in k_{g}\left(c_{1}, c_{2}, c_{3}, c_{4}\right)$. We may also consider the $d_{i}$ to be independent transcendentals contained in $k_{g}$ (here the $d_{i}$ are not the same as in Example 2.40). Let $N$ denote a square root of the norm $N_{A_{4} / k_{g}}\left(\delta^{\prime}\right)$ of $\delta^{\prime}$. Note that as always we have $\varphi_{j}=\sum_{i=0}^{5} \omega_{j}^{i} a_{i}$, where the $a_{i}$ are the original coordinates of $\mathbb{P}(A)$. The polynomials $x_{i}^{\prime \prime}, y_{i}^{\prime \prime}$, and $z_{i}^{\prime \prime}$ are quadratic in the $a_{i}$ with coefficients in the field $\mathbb{Q}\left(c_{1}, c_{2}, c_{3}, c_{4}, d_{0}, d_{1}, d_{2}, d_{3}, N\right)$. Expressing them as such takes a file that has size about one megabyte 23]. Note also that the equations for the fibration do not depend on $\omega_{5}$ and $\omega_{6}$. This shows that 
the fibration does indeed factor through the quadric $D_{\omega_{5} \omega_{6}}$ in $\mathbb{P}^{3}$ of Remark 2.5], because that is the image of $\bar{V}$ under the projection from $\mathbb{P}(\bar{A})$ to $\mathbb{P}^{3}$ using only the coordinates $\varphi_{1}, \ldots, \varphi_{4}$.

Remark 2.53. In any specific example, we can consider the specialization of the equations for $C_{3}$ and the fibration $\nu_{3}$ in Lemma 2.51, or better, Remark 2.52 For a proper closed subset in the family of all curves of genus 2 and choices of $\delta$ these equations may vanish. Outside this subset, this specialization gives us an elliptic fibration $\nu$ of a surface $V$ over a conic $C$. If $V$ is everywhere locally solvable, then so is $C$. Since $C$ satisfies the Hasse principle, this implies that $C$ has a rational point, which can be found by standard algorithms. This gives us a rational fiber $F_{0}$ on $V$. The linear system of hyperplanes through $F_{0}$ is 2-dimensional and determines an elliptic fibration $\nu^{\prime}$ of $V$ over $\mathbb{P}^{1}$, given by linear polynomials. The 4 -gons that are fibers of $\nu^{\prime}$ belong to the complementary exhibit of the exhibit whose 4-gons are fibers of $\nu$. Applying the same trick again, we also obtain an elliptic fibration over $\mathbb{P}^{1}$ that is equivalent to $\nu$. On the other hand, even if $C$ is everywhere locally solvable, $V$ may not be.

\section{Arithmetical applications}

In this section we combine the theory of the previous section with the idea of the Brauer-Manin obstruction. We will give a K3 surface $V$ on which the BrauerManin obstruction blocks the existence of rational points. We also exhibit a family of curves of genus 2 for each member of which there exists a twist of the Jacobian that has rational points everywhere locally and that admits a map to $V$. It follows that the twists do not have global points and thus that every member of the family of curves of genus 2 has nontrivial Tate-Shafarevich group.

3.1. The Brauer group and the Brauer-Manin obstruction. To explain the Brauer-Manin obstruction, we let $V$ be a variety over a number field $K$. If there is a place $\mathfrak{p}$ of $K$ at which $V$ has no points, then of course $V$ has no $K$-rational points. But if $V$ has points everywhere locally, we can sometimes use the Brauer group to prove that it does not have any points defined over $K$. We begin by defining the Brauer group of a scheme; this material is taken from the beginning of [13, Chapter 4.

Definition 3.1. Let $R$ be a ring. Then an Azumaya algebra $A$ over $R$ is a free $R$ algebra of finite rank as an $R$-module such that $A \otimes_{R} A^{\text {op }}$ is isomorphic to $\operatorname{End}(A)$ by the map taking $a \otimes a^{\prime}$ to the endomorphism $x \rightarrow a x a^{\prime}$.

Definition 3.2. Let $V$ be a scheme. An Azumaya algebra $\mathcal{A}$ on $V$ is a coherent sheaf of $\mathcal{O}_{V}$-algebras whose stalk at every point $x$ of $V$ is an Azumaya algebra over the local ring of $V$ at $x$. The Brauer group of $V$ is the semigroup of Azumaya algebras on $V$ under tensor product modulo the subsemigroup of endomorphism algebras of locally free sheaves.

The Brauer group of a field $K$ is often defined as the semigroup of finitedimensional central simple algebras over $K$ under the operation of tensor product modulo the subsemigroup $\left\{M_{n}(K): n \in \mathbb{N}\right\}$. This is a special case of the definition 
above. Alternatively, $\mathrm{Br} K$ can be thought of as $H^{2}\left(K, K^{\mathrm{sep}}{ }^{*}\right)$, which can be rewritten as $H_{\text {ét }}^{2}\left(\operatorname{Spec} K, K^{\mathrm{sep} *}\right)$ using the standard equivalence ([13], Example III.1.7) between étale cohomology of Spec $K$ and Galois cohomology of $\operatorname{Gal}\left(K^{\text {sep }} / K\right)$-modules. We extend this definition to general schemes as follows.

Definition 3.3. For a variety $V$ over a field $K$, we use $\bar{V}$ to denote $V \otimes_{K} \bar{K}$.

Definition 3.4. The cohomological Brauer group $\operatorname{Br} V$ of a scheme $V$ is $H_{\text {ét }}^{2}\left(V, \mathbb{G}_{m}\right)$. If $V$ is defined over a field $K$, then the algebraic part of the Brauer group $\mathrm{Br}_{1} V$ is the kernel of the natural map $\operatorname{Br} V \rightarrow \operatorname{Br} \bar{V}$.

We can use the Brauer group to find obstructions to the existence of points, but it is difficult to compute. However, we can compute the algebraic part of the Brauer group. By [13, Prop. IV.2.15, the Brauer group and the cohomological Brauer group are isomorphic when $V$ is a smooth variety over a field. For $V$ defined over a number field, the algebraic part of the Brauer group can be computed as $H^{1}(K, \operatorname{Pic} \bar{V})$ : this is part of [19, Cor. 2.3.9. As stated in Corollary 2.4, we have Pic $\bar{V}=\mathrm{NS} \bar{V}$, a finitely generated free abelian group.

In this paper, we will only consider the algebraic part of the Brauer group. Now we explain how to use the Brauer group to show that $V$ has no $K$-rational points.

Definition 3.5. Let $K$ be a number field and $\mathfrak{p}$ a place of $K$. The local invariant $\operatorname{inv}_{\mathfrak{p}}(s)$ of an element $s$ of the Brauer group of the local field $K_{\mathfrak{p}}$ is its image under the natural homomorphism $\operatorname{Br} K_{\mathfrak{p}} \rightarrow \mathbb{Q} / \mathbb{Z}$ ([17, Proposition XII.6).

Recall that this homomorphism is always injective, and that it is surjective if $\mathfrak{p}$ is non-archimedean, while its image is generated by $1 / 2$ if $\mathfrak{p}$ is real and is trivial if $\mathfrak{p}$ is complex.

Definition 3.6. For $s \in \operatorname{Br} V, \mathfrak{p}$ a place of $K$, and $P \in V\left(K_{\mathfrak{p}}\right)$, the local invariant of $s$ at $P$ is the local invariant $\operatorname{inv}_{\mathfrak{p}}\left(s_{P}\right)$ of the element $s_{P} \in \operatorname{Br} K_{\mathfrak{p}}$ obtained by pulling back the cohomology class $s$ by the map Spec $K_{\mathfrak{p}} \rightarrow V$ whose image is the point $P$. More concretely, it is the local invariant of the Azumaya algebra over $K_{\mathfrak{p}}$ whose multiplication table is given by evaluating the elements of the multiplication table of an Azumaya algebra representing $s$ at the coordinates of $P$.

Every $K$-point $P$ of $V$ corresponds to a morphism Spec $K \rightarrow V$ that induces a map $\operatorname{Br} V \rightarrow \operatorname{Br} K$ by pulling back cohomology. We denote the image of an element $s \in \operatorname{Br} V$ under this map by $s(P)$, yielding a map $V(K) \rightarrow \operatorname{Br} K$ that is also denoted by $s$. Similarly, for every place $\mathfrak{p}$ (archimedean or non-archimedean) of $K$ we get a map $s_{\mathfrak{p}}: V\left(K_{\mathfrak{p}}\right) \rightarrow \operatorname{Br} K_{\mathfrak{p}}$. We obtain the following commutative diagram, where the top horizontal map is the diagonal embedding and $\lambda_{s}$ is defined to be the composition shown:

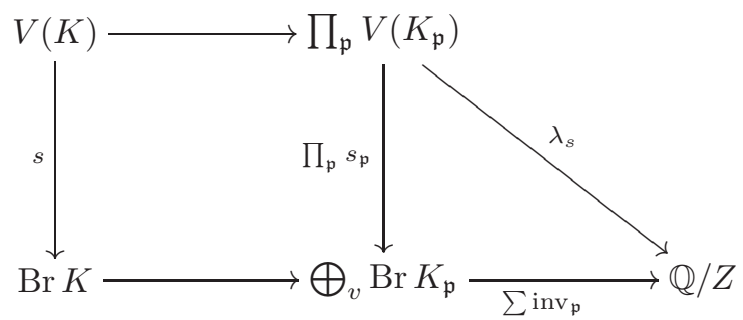


The bottom row is exact by class field theory, so the image of the top horizontal map is contained in $\lambda_{s}^{-1}(0)$. If we show that $\bigcap_{s \in \operatorname{Br} V} \lambda_{s}^{-1}(0)=\emptyset$, then we may conclude that there is no $K$-rational point on $V$, and we say that the BrauerManin obstruction blocks the existence of rational points on $V$. It suffices to let $s$ run over a set of generators of $\mathrm{Br} V / \mathrm{Br} K$. Often it is more convenient to consider only elements of $\operatorname{Br} V / \operatorname{Br} K$ belonging to a subset $B$, and then we speak of the Brauer-Manin obstruction on $B$ blocking the existence of rational points.

Proposition 3.7. Let $B$ be a subgroup of $\operatorname{Br} V / \operatorname{Br} K$ of order 2 generated by the element s. Then the Brauer-Manin obstruction on B blocks the existence of rational points on $V$ if and only if, for all $\mathfrak{p}$, the local invariant of $s$ is constant on $K_{\mathfrak{p}}$-points of $V$ and the constants do not add to 0 .

Proof. The sufficiency of this condition is clear. For the necessity, note that, if the local invariant is not constant on $K_{\mathfrak{p}}$-points for some $\mathfrak{p}$, we may choose an arbitrary collection of local points at other places with invariants adding to $\alpha \in\{0,1 / 2\}$ and then choose a $K_{\mathfrak{p}}$-point whose invariant is equal to $\alpha$, thus obtaining a system of points with local invariants adding to 0 . In the case where the local invariant is constant on $K_{\mathfrak{p}}$-points for all $\mathfrak{p}$, the necessity of the condition that the constants not add to 0 is obvious.

Now let us explain how to construct K3 surfaces with elements of the Brauer group that are likely to give nontrivial obstructions. Although it is usually easy to calculate $H^{1}(K, \operatorname{Pic} \bar{V})$, at least when $H^{1}\left(V, \mathcal{O}_{V}\right)=0$ and so Pic $\bar{V}$ is finitely generated, it is difficult to find Azumaya algebras corresponding to nontrivial cohomology classes. Doing so requires finding rational divisors in rational divisor classes. Such divisors always exist if $V$ has points everywhere locally, but in practice it is not easy to find them. Over an algebraically closed field the effective divisors in a linear equivalence class constitute a $\mathbb{P}^{n}$, so the problem reduces to finding a rational point on a locally solvable Brauer-Severi variety. This can be reduced to solving a norm equation from a field over which all elements of Pic $\bar{V}$ are defined, but even when the splitting field of $f$ is fairly small this is likely to be impractical.

We will follow [4, section 4.4, in using elliptic fibrations on varieties to construct nontrivial elements of the Brauer group. For a variety with a fibration $\phi$, we define the vertical Picard group $\operatorname{Pic}_{\phi} \bar{V}$ to be the subgroup of Pic $\bar{V}$ spanned by the classes of components of fibers. The vertical Brauer group $\operatorname{Br}_{\phi} V$ can then be defined as $H^{1}\left(K, \mathrm{Pic}_{\phi} \bar{V}\right)$. (We deviate from the standard notations $\mathrm{Pic}_{\text {vert }}$ and $\mathrm{Br}_{\text {vert }}$ used in [4 because it is necessary for us to distinguish between vertical Picard and Brauer groups coming from different fibrations on the same variety.) The inclusion of $\operatorname{Pic}_{\phi} \bar{V}$ into Pic $\bar{V}$ gives a natural map $\operatorname{Br}_{\phi} V \rightarrow H^{1}(K, \operatorname{Pic} \bar{V})$, which need not be either injective nor surjective.

It is shown in 4, Prop. 4.21, that elements of $\mathrm{Br}_{\phi} V$ are represented by Azumaya algebras that are pulled back from central simple algebras on the function field of the target of $\phi$. The stalk of such an Azumaya algebra is constant on all fibers of $\phi$, so the local invariants of such algebras are too.

Definition 3.8. Let $F$ be any field, and $a$ and $b$ nonzero elements of $F$. The symbol $(a, b)$ denotes the central simple $F$-algebra of rank 4 with basis $1, i, j, k$ and multiplication given by $i^{2}=a, j^{2}=b, i j=k, j i=-k$.

Proposition 3.9. If $F$ is a local field, the local invariant of $(a, b)$ is 0 if and only if the quadratic form $x^{2}-a y^{2}-b z^{2}$ represents 0 nontrivially in $F$; otherwise it is 
$1 / 2$. In particular, if the residue characteristic of $F$ is odd, then the invariant is 0 if and only if at least one of the following conditions holds: $a$ and $b$ both have even valuation; one of $a$ and $b$ is a square; or $a b$ is a square.

Proof. The first statement is well known (note in particular that if $a$ or $b$ is a square, then the local invariant is 0 ). The second follows from the discussion at the beginning of [17, section XIV.4, which applies just as well to any finite extension of $\mathbb{Q}_{p}$ as to $\mathbb{Q}_{p}$ itself.

Proposition 3.10. Let $\phi: V \rightarrow \mathbb{P}^{1}$ be an elliptic fibration, and suppose that $V$ has bad fibers of type $I_{4}$ over $(\alpha: 1)$, where $[\mathbb{Q}(\alpha): \mathbb{Q}]=4$. Suppose further that the field of definition of the components of the fiber at $(\alpha: 1)$ is $\mathbb{Q}(\alpha, \sqrt{c})$, where $c \in \mathbb{Q}(\alpha)$ is of square norm, and that the $\mathbb{Q}(\alpha)$-components of this fiber consist of two disjoint lines. Then the pullback of the algebra cores $\mathbb{Q}(\alpha) / \mathbb{Q}(c, t-\alpha) \in \operatorname{Br} \mathbb{Q}(t)$ to $V$, where $t$ is the coordinate on the standard affine patch of $\mathbb{P}^{1}$, is an element of $\mathrm{Br}_{\phi} V$.

Proof. This follows immediately from [4, Proposition 4.28.

Remark 3.11. Note that the hypotheses entail that the fiber consists of two pairs of disjoint lines $L_{1}, M_{1}$ and $L_{2}, M_{2}$, where all the $L_{i}$ and $M_{i}$ are defined and conjugate over one and the same quadratic extension $\mathbb{Q}(\alpha, \sqrt{c})$ of $\mathbb{Q}(\alpha)$.

For this to be useful to us, we need to know how to compute the local invariants of the algebra $\operatorname{cores}_{\mathbb{Q}(\alpha) / \mathbb{Q}}(c, t-\alpha) \in \operatorname{Br} \mathbb{Q}$. The following proposition addresses this question.

Proposition 3.12 (cf. [5], Lemma 5 (i)). The local invariant of $\operatorname{cores}_{\mathbb{Q}(\alpha) / \mathbb{Q}}(c, t-\alpha)$ at $p$ is the sum of those of $(c, t-\alpha)$ at places of $\mathbb{Q}(\alpha)$ lying above $p$.

Now we are ready to show how we chose $f$ and $\delta$ so that there would be a nontrivial element of the Brauer group arising from an elliptic fibration.

Definition 3.13. From now on, $f$ will always denote a polynomial which is the product of three irreducible quadratic polynomials $f_{1}, f_{2}, f_{3}$ and $\delta$ will be an element of $(\mathbb{Q}[X] /(f))^{*}$. For given $f$ and $\delta$, let $\iota$ be an isomorphism from $\mathbb{Q}[X] /(f)$ to $\bigoplus_{i=1}^{3} \mathbb{Q}[X] /\left(f_{i}\right)$ (which exists by the Chinese Remainder Theorem), fix isomorphisms $\kappa_{i}$ from $\overline{\mathbb{Q}}[X] /\left(f_{i}\right)$ to $\overline{\mathbb{Q}} \oplus \overline{\mathbb{Q}}$ (again by the Chinese Remainder Theorem), let $v_{i}$ be the component of $\iota(\delta)$ in $\mathbb{Q}[X] /\left(f_{i}\right)$, and let $v_{i k}$ be the $k$ th component of $\kappa_{i}\left(v_{i}\right)$. For convenience define $\delta_{j}$ so that $\delta_{2(i-1)+k}=v_{i k}$ (this notation coincides with the $\delta_{j}$ of Example 2.40). Also, let $\sigma_{i}$ be the nontrivial automorphism of $\mathbb{Q}[X] /\left(f_{i}\right)$ and let $r_{i}$ be a fixed root of $f_{i}$ in $\overline{\mathbb{Q}}$.

Theorem 3.14. With $f$ and $\delta$ as above, let $V$ be the K3 surface constructed from $f, \delta$. Suppose further that the splitting field of $f$ is of degree 8 ; that the norm of $v_{1}$ is a square; that the norms of $v_{2}$ and $v_{3}$ multiplied by the discriminant of $f_{1}$ are squares; and that the $v_{i}$ are otherwise generic. Then the field of definition of the lines of $V$ has degree 32, both elliptic fibrations associated to the factorization $f=\left(f_{1}\right)\left(f_{2} f_{3}\right)$ in Remark 2.36 satisfy the conditions of Proposition 3.10, and the elements of the respective vertical Brauer groups constructed in that proposition map to the same element of $H^{1}(\mathbb{Q}, \operatorname{Pic} \bar{V})$ and hence to the same element of $\operatorname{Br} V / \operatorname{Br} K$.

Proof. The condition on $\delta$ shows that the norm of the projection of $\delta$ to $\mathbb{Q}[X] /\left(f_{2} f_{3}\right)$ is a square, so the elliptic fibrations are defined over $\mathbb{Q}$ by Remark 2.36. As we 
did in the discussion just after Lemma 2.32, fix a square root $\sqrt{\delta_{j}}$ of $\delta_{j}$ for $j \in$ $\{1, \ldots, 6\}$ (corresponding to $\epsilon_{j}$ in Example 2.40). Let us now determine the action of the absolute Galois group of $\mathbb{Q}$ on the lines. By Lemma 2.38, it factors through the extension $m$ of the splitting field $l=\mathbb{Q}\left(r_{1}, r_{2}, r_{3}\right)$ of $f$ obtained by adjoining all elements of the form $\sqrt{\delta_{i}} \sqrt{\delta_{j}}$. Note that $\delta_{1} \delta_{2}=v_{11} v_{12}=N_{\left(\mathbb{Q}[X] / f_{1}\right) / \mathbb{Q}}\left(v_{1}\right)$ is a square by hypothesis. Similarly, letting $\Delta_{1}$ denote the discriminant of $f_{1}$, we find that $\Delta_{1} \delta_{3} \delta_{4}$ and $\Delta_{1} \delta_{5} \delta_{6}$ are squares, and thus that $\delta_{3} \delta_{4}$ and $\delta_{5} \delta_{6}$ are squares in $\mathbb{Q}\left(\sqrt{\Delta_{1}}\right)=\mathbb{Q}\left(r_{1}\right)$. It follows that up to square factors in $\mathbb{Q}\left(r_{1}\right)$ every element of the form $\delta_{i} \delta_{j}$ is equivalent to $\delta_{1} \delta_{3}, \delta_{1} \delta_{5}$, or $\delta_{3} \delta_{5}$. We conclude that $m=l\left(\sqrt{\delta_{1}} \sqrt{\delta_{3}}, \sqrt{\delta_{1}} \sqrt{\delta_{5}}\right)$. As the $v_{i}$ are otherwise generic, we find that the field $m$ of definition of the lines has degree 4 over $l$ and therefore degree 32 over $\mathbb{Q}$.

We now describe the Galois group of $m$ over $\mathbb{Q}$ in terms of the $s, t$ described in the discussion following Proposition 2.41. The $v_{i}$ are generic aside from their given properties, so the field $m^{\prime}=l\left(\sqrt{\delta_{1}}, \ldots, \sqrt{\delta_{6}}\right)=m\left(\sqrt{\delta_{1}}\right)$ has degree 2 over $m$ (cf. discussion following Lemma 2.32 and Example 2.40). Recall that for a permutation $p \in S_{6}$, the automorphism $t_{p}$ of $m$ is induced from $m^{\prime}$ by sending $\delta_{j}$ and $\sqrt{\delta_{j}}$ to $\delta_{p(j)}$ and $\sqrt{\delta_{p(j)}}$ respectively. For a subset $I \subseteq\{1,2, \ldots, 6\}$, the automorphism $s_{I}$ of $m$ is induced from $m^{\prime}$ by fixing all $\delta_{j}$ and sending $\sqrt{\delta_{j}}$ to $\pm \sqrt{\delta_{j}}$ where the sign is negative if and only if $j \in I$. We will also refer to the 32 lines on $V$ using the notation $L_{I}$ introduced before Lemma 2.33 .

The automorphism $\sigma_{1}$ lifts to an automorphism of $m^{\prime}$ that fixes $r_{2}$ and $r_{3}$, and therefore all $\delta_{j}$ for $3 \leq j \leq 6$. Because $\sigma_{1}$ sends $\sqrt{\Delta_{1}}$ to $-\sqrt{\Delta_{1}}$, and $\delta_{3} \delta_{4}$ and $\delta_{5} \delta_{6}$ are squares up to a factor $\Delta_{1}$, this lift changes the signs of the square roots of one of $\delta_{3}$ and $\delta_{4}$ and of one of $\delta_{5}$ and $\delta_{6}$. The induced automorphism of $m$ is then $t_{(12)} s_{i, j}$ for some $i \in\{3,4\}$ and $j \in\{5,6\}$. The automorphisms $\sigma_{2}$ and $\sigma_{3}$ lift to $t_{(34)}$ and $t_{(56)}$ respectively. Together with $s_{\{1,2\}}$ and $s_{\{3,4\}}$ these elements generate a subgroup of $\operatorname{Gal}(m / \mathbb{Q})$ of order 32 , so they generate the full Galois group. The orbits of this group on the lines are of order 8 and each orbit contains two nonintersecting lines from each of the four fibers of one fibration. In particular, all four $I_{4}$ fibers are conjugate, so they are indeed defined over a field of degree 4 .

By Remark 2.36 the fibers of the elliptic fibrations are orbits of $\Lambda$ under the group generated by $s_{1}$ and $s_{2}$ in Aut $\Lambda$ (only their product $s_{1} s_{2}$ is in the subgroup of Aut $\Lambda$ induced by Galois). Consider first the fibration associated to the exhibit $\mathcal{S}$ that contains a 4-gon $S$ containing $L_{0}$. Then that 4-gon is $S=\left\{L_{0}, L_{1}, L_{2}, L_{12}\right\}$. One easily checks that the subgroup $\operatorname{Gal}(m / k(S))$ is generated by $t_{(34)}, t_{(56)}$, and $s_{\{1,2\}}$. This group fixes $\mathbb{Q}\left(r_{1}\right)$, so we have $\mathbb{Q}\left(r_{1}\right) \subset k(S)$, and in fact the group $\operatorname{Gal}\left(m / \mathbb{Q}\left(r_{1}\right)\right)$ is generated by $\operatorname{Gal}(m / k(S))$ and $s_{\{3,4\}}$. Under $\operatorname{Gal}(m / k(S))$ the 4-gon $S$ breaks up into the orbits $\left\{L_{0}, L_{12}\right\}$ and $\left\{L_{1}, L_{2}\right\}$, each consisting of two disjoint lines.

Let $L$ be any line in $S$. The subgroup $\operatorname{Gal}(m / k(L))$ is generated by $t_{(34)}$ and $t_{(56)}$, which is normal in $\operatorname{Gal}\left(m / \mathbb{Q}\left(r_{1}\right)\right)$. Therefore, $k(L)$ is Galois over $\mathbb{Q}\left(r_{1}\right)$ and the corresponding Galois group is $(\mathbb{Z} / 2 \mathbb{Z})^{2}$. Since $k(S)$ is one of the quadratic subfields, it follows from elementary Galois theory that $k(L)$ can be obtained from $k(S)$ by adjoining the square root of an element $c \in \mathbb{Q}\left(r_{1}\right)$. As $k(S)$ has degree 4 over $\mathbb{Q}$ and $\mathbb{Q}\left(r_{1}\right)$ is a quadratic subextension, the norm of $c$ from $k(S)$ to $\mathbb{Q}$ is indeed a square. The arguments for the opposite fibration are completely similar.

The final statement of the proposition, that the elements of the vertical Brauer group obtained in this way give the same element of $H^{1}(\mathbb{Q}, \operatorname{Pic} \bar{V})$, is proved by a 
calculation using MAGMA. The point is to verify that the images of the nontrivial elements of $H^{1}\left(\mathbb{Q}, \operatorname{Pic}_{\phi} V\right)$ and $H^{1}\left(\mathbb{Q}, \operatorname{Pic}_{\phi^{\prime}} V\right)$ constructed in Proposition 3.10 in $H^{1}(\mathbb{Q}, \operatorname{Pic} \bar{V})$ are equal, where $\phi$ and $\phi^{\prime}$ are the two fibrations associated to the factorization $f=\left(f_{1}\right)\left(f_{2} f_{3}\right)$ as in Remark 2.36, See [23, for details.

Remark 3.15. In special cases it is possible for the Picard group of $V$ to have higher rank than in the generic case (in fact this happens in the example that we present immediately below). Thus we may have constructed elements, not of $H^{1}(\mathbb{Q}, \operatorname{Pic} \bar{V})$, but only of $H^{1}(\mathbb{Q}, P)$, where $P$, the subgroup of Pic $\bar{V}$ generated by the classes of the lines, is a proper subgroup of Pic $\bar{V}$. The inclusion $P \rightarrow \operatorname{Pic} \bar{V}$ gives a map from $H^{1}(\mathbb{Q}, P)$ to $H^{1}(\mathbb{Q}$, Pic $\bar{V})$, which allows us to consider the elements we have constructed as elements of the Brauer group. It is possible that our elements could be in the kernel of this map in some situations. However, they are well-defined Brauer classes, and so they may be used to attempt to prove that $V$ has no rational points. If their local invariants do not add to 0 , it indicates that they are not in the kernel.

Remark 3.16. It is not essential for the method that the element of $H^{1}(\mathbb{Q}, \operatorname{Pic} \bar{V})$ be obtainable from two different fibrations. However, as was first pointed out by Swinnerton-Dyer, this means that the local invariants are constant on the fibers of two different fibrations and are therefore much more likely to be constant than would otherwise be the case. This greatly facilitated finding the example below.

3.2. An explicit example. Now let us present our example, which was found by searching various choices of $f_{1}, f_{2}, f_{3}$ with small splitting fields $\mathbb{Q}(i), \mathbb{Q}(\sqrt{2}), \mathbb{Q}(\sqrt{5})$ such that the discriminant of $f_{1} f_{2} f_{3}$ is small and $\delta$ such that the field of definition of the lines would not introduce new bad primes. For the rest of this paper, let $f=\left(x^{2}+1\right)\left(x^{2}-2 x-1\right)\left(x^{2}+x-1\right)=f_{1}(x) f_{2}(x) f_{3}(x)$, let $A_{f}=\mathbb{Q}[X] / f(X)$, let $E$ be the algebra $\mathbb{Q}(i) \oplus \mathbb{Q}(\sqrt{2}) \oplus \mathbb{Q}(\sqrt{5})$, fix an isomorphism $\iota: A_{f} \rightarrow E$ as in Definition 3.13, and let

$$
\delta=\left(-2 X^{5}+3 X^{4}+5 X^{3}-8 X^{2}+7 X+7\right) / 6 \in A_{f},
$$

so that $\iota(\delta)=(3,-(1+\sqrt{2}),(1+\sqrt{5}) / 2)$. Then $f, \delta$ satisfy the conditions of Theorem 3.14. For nonzero rational $t$ let $C_{t}$ be the curve $y^{2}=t f(x)$. Let $V$ be the $\mathrm{K} 3$ surface $V_{f, \delta}$. Our goal is to prove the main theorem, Theorem 1.1 restated and slightly reworded here for ease of reading:

Theorem 3.17. Let $S$ be the union of $\{5\}$ with the set of primes that split completely in the field of definition of the lines of $V$, which is

$$
F=\mathbb{Q}(\sqrt{-1}, \sqrt{2}, \sqrt{5}, \sqrt{-3(1+\sqrt{2})}, \sqrt{6(1+\sqrt{5})}) .
$$

Then for all $n$ that are products of elements of $S$, the 2-part of the Tate-Shafarevich group of the Jacobian of the curve $C_{-6 n}$ is nontrivial.

To do so, we will follow the strategy outlined in the introduction: First we will show that $\delta$ gives an element of the fake Selmer group of the Jacobian of the curve in question by showing that the corresponding principal homogeneous space of the Jacobian of $C_{-6 n}$ has points everywhere locally. Then we will use the element of the Brauer group described above to prove that $V_{f, \delta}$ has no rational points and conclude that the principal homogeneous space has no rational points either. We begin by 
summarizing some results from [20] on the fake Selmer group of the Jacobian of a hyperelliptic curve of genus 2 .

Definition 3.18. Let $g$ be a squarefree polynomial of degree 6 over $\mathbb{Q}$ and $C$ the curve $y^{2}=g(x)$. For any field $K$ of characteristic 0 , let $H_{K}=\operatorname{ker}\left(N:\left(A_{g} \otimes\right.\right.$ $\left.K)^{*} /\left(A_{g} \otimes K\right)^{* 2} K^{*} \rightarrow K^{*} / K^{* 2}\right)$ with $A_{g}=\mathbb{Q}[X] / g(X)$. (Note that the norm is well defined, because $\operatorname{deg} g$ is even and so $N\left(K^{*}\right) \subset K^{* 2}$.) Write $H$ instead of $H_{\mathbb{Q}}$. As in [20, Prop. 5.5, let the fake Selmer group $\operatorname{Sel}_{\text {fake }}^{(2)}(\mathbb{Q}, \mathrm{Jac} C)$ of $C$ be the subgroup of $H$ consisting of elements that are everywhere locally in the image of the 2-descent map on the Jacobian of $C$. Define $\Delta_{K}$, the descent map over $K$, to be the function from the set of $K$-rational points of $C$ which are neither Weierstrass points nor points at infinity to $H_{K}$ such that $\Delta_{K}\left(x_{0}, y_{0}\right)=1 \otimes x_{0}-X \otimes 1$.

Proposition 3.19. The function $\Delta_{K}$ may be extended multiplicatively to a function from $J(K)$ to $H_{K}$.

Proof. This is essentially [16, Lemma 2.1 , as modified by the discussion in section 2.5 ; see also 20, sect. 6 for explicit formulas for Weierstrass points.

Proposition 3.20. Let $S$ be the set of primes introduced in Theorem 3.17. Then for all $n$ that are products of elements of $S$, the fake Selmer group of the Jacobian of $C_{-6 n}$ contains $\delta$. Equivalently, the principal homogeneous space of $\operatorname{Jac}\left(C_{-6 n}\right)$ corresponding to $\delta$ is everywhere locally solvable.

Proof. We need only show that $\delta$ is in the image of the local Selmer maps for primes of bad reduction and $\infty$. The primes of bad reduction are 2, 3, 5, and primes dividing $n$. At a prime $p$ dividing $n$, we have that $\delta$ corresponds under $\iota$ to an element of the form $\left(3,3 a^{2}, 3 b^{2}\right)$ in $E \otimes \mathbb{Q}_{p}$. Therefore, $\delta$ is contained in $\left(A_{f} \otimes \mathbb{Q}_{p}\right)^{*} \mathbb{Q}_{p}^{*}$, so it is the identity element in $H_{\mathbb{Q}_{p}}$ and is in the image of every homomorphism. Thus $\delta$ is in the image of the local 2-descent map. Note also that every product of elements of $S$ is positive, is equivalent to 1 or 5 in $\mathbb{Q}_{p}^{*} / \mathbb{Q}_{p}^{* 2}$ for $p=2,5$, and is a unit locally at 3 . It follows that we need only check the assertion for $n=1, p=2,3,5, \infty$, and $n=5, p=2,3,5$. This can be done by using MAGMA's TwoSelmerGroup command to compute the fake Selmer groups of $C_{-6}$ and $C_{-6.5}$ and verifying that $\delta$ is an element of both. The last statement follows by applying the fact that the principal homogeneous space over a field $K$ corresponding to $\delta$ has points everywhere locally if and only if $\delta$ is in the image of $\Delta_{K}$ to all completions of $\mathbb{Q}$.

Proposition 3.21. If the image of $\operatorname{Jac}\left(C_{t}\right) / 2 \operatorname{Jac}\left(C_{t}\right)$ under the 2-descent map $\Delta_{\mathbb{Q}}$ is properly contained in the fake Selmer group, then $\operatorname{Jac}\left(C_{t}\right)$ has nontrivial TateShafarevich group.

Proof. The map $\operatorname{Jac}\left(C_{t}\right) / 2 \operatorname{Jac}\left(C_{t}\right) \rightarrow \operatorname{Sel}_{\text {fake }}^{(2)} \operatorname{Jac}\left(C_{t}\right)$ factors through the surjective map from the actual Selmer group $\operatorname{Sel}^{(2)}\left(\operatorname{Jac}\left(C_{t}\right)\right)$ to $\operatorname{Sel}_{\text {fake }}^{(2)} \operatorname{Jac}\left(C_{t}\right)$. Therefore the image of $\operatorname{Jac}\left(C_{t}\right) / 2 \mathrm{Jac}\left(C_{t}\right)$ in $\operatorname{Sel}^{(2)}\left(\mathrm{Jac}\left(C_{t}\right)\right)$ is also properly contained in $\operatorname{Sel}^{(2)}\left(\operatorname{Jac}\left(C_{t}\right)\right)$. Every element of $\operatorname{Sel}^{(2)}\left(\operatorname{Jac}\left(C_{t}\right)\right)$ not in the image, maps to a nontrivial element of the Tate-Shafarevich group. 
Now we indicate the relation between the descent map and the K3 surface $V_{f, \delta}$ that we have defined.

Proposition 3.22. With $f$ and $\delta$ as above, let $V$ be the $K 3$ surface constructed from $f, \delta$. Suppose that $V$ has no rational points. Then $\delta$ is not in the image of the 2-descent map for the Jacobian of the curve $y^{2}=f(x)$.

Proof. Suppose, to the contrary, that $\delta$ were in the image; that is, that $\Delta_{\mathbb{Q}}(D)=\delta$ for some rational divisor $D$ of degree 0 . By Riemann-Roch, this divisor may be taken to be of the form $\left(x_{1}, y_{1}\right)+\left(x_{2}, y_{2}\right)-H$, where $H$ is the hyperelliptic divisor. Since $\Delta_{\mathbb{Q}}(H)=1$, this means that $\Delta_{\mathbb{Q}}(D)=\Delta_{\mathbb{Q}}\left(\left(x_{1}, y_{1}\right)+\left(x_{2}, y_{2}\right)\right)=\left(x_{1}-X\right)\left(x_{2}-\right.$ $X)$ in $A_{f}^{*} /\left(A_{f}^{*}\right)^{2} \mathbb{Q}^{*}$, where we have identified $A_{f} \otimes_{\mathbb{Q}} \mathbb{Q}$ with $A_{f}$. In other words, for some $r \in \mathbb{Q}$ and $q \in A_{f}$ we have $\delta q^{2}=r x_{1} x_{2}-r\left(x_{1}+x_{2}\right) X+r X^{2}$. In particular, the point of $\mathbb{P}^{5}$ whose coordinates are the coefficients of $q$ lies on $V$.

Remark 3.23. Propositions 3.21 and 3.22 together imply that if $\delta$ is an element of the fake Selmer group $\operatorname{Sel}_{\text {fake }}^{(2)}\left(\mathbb{Q}, \operatorname{Jac} C_{t}\right)$ and the corresponding $V_{f, \delta}$ has no rational points, then $\operatorname{Jac}\left(C_{t}\right)$ has nontrivial Tate-Shafarevich group. This also follows from the fact that $V_{f, \delta}=V_{t f, \delta}$ is a quotient of the homogeneous space of Jac $C_{t}$ corresponding to $\delta$. Indeed, this implies that if $V_{f, \delta}$ has no rational points, then neither does the homogeneous space, which implies that its image in the Tate-Shafarevich group is nontrivial.

It is also worth mentioning that the existence of the nontrivial element of $\amalg$ that we will exhibit is not a consequence of the results of [15] on odd curves, which we now describe briefly.

Definition $3.24([15)$. Let $C$ be a curve over $\mathbb{Q}$ of genus $g$. We say that $C$ is deficient at $p$, where $p$ is a prime or $\infty$, if $C$ has no rational divisor of degree $g-1$ over $\mathbb{Q}_{p}$. We say that $C$ is even or odd depending on the parity of the number of places at which $C$ is deficient.

Denote the 2-primary part of $\amalg(\operatorname{Jac}(C))$ by $\amalg(\operatorname{Jac}(C))\left[2^{\infty}\right]$. Then, if $\amalg(\operatorname{Jac}(C))\left[2^{\infty}\right]$ is finite, its order is a square if and only if $C$ is even ([15, Theorem 11). It follows that if $C$ is odd, then $\amalg(\operatorname{Jac}(C))\left[2^{\infty}\right]$ is nontrivial. The following proposition proves that the elements of $\amalg$ that we find are not merely artifacts of the oddness of our curves.

Proposition 3.25. If $n$ is a product of primes in $S$, then $C_{-6 n}$ is even.

Proof. A curve is never deficient at a prime $p$ of good reduction, for there are always points over all sufficiently large finite fields of characteristic $p$ and therefore over unramified extensions of $\mathbb{Q}_{p}$ of all sufficiently large degrees. The primes of bad reduction are $2,3,5$, and those dividing $n$. The point $(i, 0)$ on $C_{-6 n}$ is defined over $\mathbb{Q}_{p}$ for all $p$ congruent to $1 \bmod 4$, which includes 5 and all primes dividing $n$. It is also clear that there are real points on $C_{-6 n}$. As a result, the only primes that need to be considered are 2 and 3. One checks (for example, using the IsDeficient command in MAGMa) that $C_{-6 n}$ is deficient at those primes. It follows that $C_{-6 n}$ is even. 
We now start to show that $V$ has no rational points, from which it follows that $\delta$ is not in the image of the global 2-descent map. First we will limit the set of primes to be considered in the calculation of the Brauer-Manin obstruction, then we will explain how to calculate the invariants there, and after that we will actually compute the invariants.

Lemma 3.26. Let $W$ be a variety over $\mathbb{Q}$ with good reduction at $p$ and let $s \in \operatorname{Br} W$. Then the local invariant of $s$ at $p$ is constant.

Proof. This is a weaker version of Theorem 1 of [5].

Lemma 3.27. Let $W$ be an elliptic surface over $\mathbb{Q}$ with an Azumaya algebra s given by $\operatorname{cores}(c, t-\alpha)$ as in Proposition 3.10. Let $p$ be a prime of good reduction for $W$ such that the fiber at infinity of $W$ has smooth $\mathbb{Q}_{p}$-rational points (in particular, this is true if the fiber at infinity has good reduction mod $p$ ). Then the local invariant of $s$ at $p$ is equal to 0 .

Proof. By Lemma 3.26, the local invariant is constant, so it suffices to evaluate it at one point. Since the fiber at infinity has smooth $\mathbb{Q}_{p}$-rational points, so do all sufficiently near fibers. In particular, for all sufficiently large integers $k$ the fiber at $p^{-2 k}$ has rational points, and the local invariant is therefore

$$
\operatorname{cores}\left(p^{-2 k}-\alpha, c\right)=\sum_{\mathfrak{p} \mid p}\left(p^{-2 k}-\alpha, c\right)_{\mathfrak{p}} .
$$

But $p^{-2 k}-\alpha$ is a square in all completions at primes above $p$ for all sufficiently large $k$, and hence the local invariant is 0 for sufficiently large $k$.

Lemma 3.28. Let $W$ be an elliptic surface over $\mathbb{Q}$ with an Azumaya algebra $\operatorname{cores}(c, t-\alpha)$, where $c$ has square norm. Suppose that the valuation of $\alpha / 4 p^{n}$ is positive at all primes above $p$ at which $c$ is not a square. Then the invariant at $p$ is 0 on all fibers of coordinate $t$ where $v(t) \leq n$.

Proof. We notice that $t /(t-\alpha)$ is a square for all $t$ with $v(t) \leq n$, and therefore that the invariants of $(c, t)$ and $(c, t-\alpha)$ are equal at all primes above $p$ where $c$ is not a square. They are also equal at primes above $\mathfrak{p}$ at which $c$ is a square, being both equal to 0 there, so they are equal at all primes above $p$. Thus the invariant at $p$ of $\operatorname{cores}(c, t-\alpha)$ is equal to that of $\operatorname{cores}(c, t)$ (see Proposition 3.12). But this is equal to the invariant of $(N(c), t)$, which is 0 because $N(c)$ is a square.

Lemma 3.29. Let $t_{0} \in \mathbb{Q}_{p}$ and $n \in \mathbb{Z}$ be such that $p^{n} / 4\left(t_{0}-\alpha\right)$ has positive valuation in $\mathbb{Q}(\alpha)_{\mathfrak{p}}$ for all primes $\mathfrak{p}$ above $p$ where $c$ is not a square locally. Then the local invariant of the Azumaya algebra $\operatorname{cores}(c, t-\alpha)$ is the same for all values of $t$ in the disc $t_{0}+p^{n} \mathbb{Z}_{p}$.

Proof. It is sufficient to show that $(t-\alpha) /\left(t_{0}-\alpha\right)$ is a square for all $t \in t_{0}+p^{n} \mathbb{Z}_{p}$ at all primes $\mathfrak{p}$ above $p$ where $c$ is not a square. But this is equal to $1+k p^{n} /\left(t_{0}-\alpha\right)$ for some $k \in \mathbb{Z}_{p}$, which by assumption is congruent to $1 \bmod 4 \mathfrak{p}$. It is therefore a square by Hensel's lemma. 
Lemma 3.30. It can be effectively determined whether there are $\mathbb{Q}_{p}$-rational points of $V$ mapping under a given fibration to a given disc $D$ in $\mathbb{P}^{1}\left(\mathbb{Q}_{p}\right)$.

Proof. By changing coordinates, we may assume that the disc is $\left\{\left(\mathbb{Z}_{p}: 1\right)\right\}$. Consider the graph of the fibration as a subscheme of $\mathbb{P}^{5} \times \mathbb{P}^{1}$. It is sufficient to determine whether there is a standard affine patch $\mathbb{A}^{5}$ of $\mathbb{P}^{5}$ such that the intersection of the patch $\mathbb{A}^{5} \times D$ of $\mathbb{P}^{5} \times \mathbb{P}^{1}$ with the graph of the fibration has $\mathbb{Z}_{p}$-points. Since the graph is smooth, this can be decided using Hensel's lemma.

Theorem 3.31. Let $V$ be a smooth variety with an elliptic fibration $\phi$ over $\mathbb{P}^{1}$ satisfying the hypotheses of Proposition 3.10 and let $\operatorname{cores}(c, t-\alpha) \in \operatorname{Br} \phi$ be the Azumaya algebra constructed there. Let $p$ be a prime. Then the set of values of the local invariant of $\operatorname{cores}(c, t-\alpha)$ on $V\left(\mathbb{Q}_{p}\right)$ can be effectively determined.

Proof. Using Hensel's lemma or Lemma 3.30 we can check whether $V$ has any $\mathbb{Q}_{p^{-}}$ points. If not, then we are done, so we may assume that $V$ has $\mathbb{Q}_{p}$-points. If $V$ has good reduction at $p$, then by Lemma 3.26 the local invariant cores $(c, t-\alpha)$ is constant, so it suffices to evaluate it at one point of $V$. Since $V$ is nonsingular, its $\mathbb{Q}_{p}$-points are dense, so we can find a point for which $t-\alpha$ is bounded away from 0 and $\infty$. It is then easy to compute the local invariant there.

We will describe the algorithm for calculating the values of the local invariant of $\operatorname{cores}(c, t-\alpha)$ at a prime $p$ of bad reduction for $V$ and then prove that it terminates. The point is that this local invariant turns out to be locally constant. In fact, we will show how to find an explicit finite covering of $\mathbb{P}^{1}\left(\mathbb{Q}_{p}\right)$ by discs in the $p$-adic topology, such that for each disc the invariant is constant on the set of $\mathbb{Q}_{p}$-points on $V$ mapping to that disc under the elliptic fibration.

First we show that such a disc exists around each point in $\mathbb{P}^{1}\left(\mathbb{Q}_{p}\right)$, starting with the point at infinity. After a change of variables we may assume that the fiber at $t=\infty$ is smooth over $\mathbb{Q}_{p}$. If the fiber at $\infty$ does have points over $\mathbb{Q}_{p}$, then by Lemma 3.28 we can find an $n$ such that the local invariant is 0 on all points on fibers above $t$ with $v(t)<n$. In this case the disc $v(t)<n$ has the desired property. If the fiber at $t=\infty$ does not have any $\mathbb{Q}_{p}$-points, then there is a $p$-adic neighborhood of $\infty \in \mathbb{P}^{1}\left(\mathbb{Q}_{p}\right)$ above which there are no $\mathbb{Q}_{p}$-points either, so the invariant is clearly constant above any disc contained in this neighborhood.

Now observe that, by Lemma 3.29, every finite $t_{0}$ has a suitable neighborhood except for those that are roots of the minimal polynomial of $\alpha$, so we are reduced to considering the finite set of these $t_{0}$. Here the argument will be more subtle. Fix such $t_{0}$ and let $\mathfrak{p}$ be the corresponding place of $\mathbb{Q}(\alpha)$, so that the completion map $\rho: \mathbb{Q}(\alpha) \hookrightarrow \mathbb{Q}(\alpha)_{\mathfrak{p}}$ sends $\alpha$ to $t_{0}$. Here we used the obvious embedding $\mathbb{Q}_{p} \hookrightarrow \mathbb{Q}(\alpha)_{\mathfrak{p}}$, which is an isomorphism as $\alpha$ is contained in $\mathbb{Q}_{p}$.

First consider the case that the fiber above $t=t_{0}=\rho(\alpha)$ does have a point $P$ over $\mathbb{Q}_{p} \cong \mathbb{Q}(\alpha)_{\mathfrak{p}}$. Then $P$ must be a smooth point of the $\mathbb{Q}(\alpha)_{\mathfrak{p}}$-component it lies on, because by hypothesis each $\mathbb{Q}(\alpha)$-component is smooth. Therefore the geometric component on which $P$ lies is defined over $\mathbb{Q}(\alpha)_{\mathfrak{p}} \cong \mathbb{Q}_{p}$, so by hypothesis all geometric components of the fiber at $t=t_{0}=\rho(\alpha)$ are defined over $\mathbb{Q}_{p}$, which means that $\rho(c)$ is a square. For all other places $\mathfrak{q}$ above $p$ the embedding $\mathbb{Q}_{p} \hookrightarrow$ $\mathbb{Q}(\alpha)_{\mathfrak{q}}$ does not send $t_{0}$ to $\alpha$, so we can find an integer $n$ such that under each such embedding the element $p^{n} / 4\left(t_{0}-\alpha\right)$ has positive valuation in $\mathbb{Q}(\alpha)_{\mathfrak{q}}$. By Lemma 3.29 
the invariant is constant above the disc $t_{0}+p^{n} \mathbb{Z}_{p}$. In the implementation of this algorithm it will be useful to remember that $c$ is a square in $\mathbb{Q}(\alpha)_{\mathfrak{p}}$, as this implies that the local invariant corresponding to the place $\mathfrak{p}$ is 0 globally. In the case that the fiber above $t=t_{0}$ does not have any $\mathbb{Q}_{p}$-points, there is a $p$-adic neighborhood of $t_{0} \in \mathbb{P}^{1}\left(\mathbb{Q}_{p}\right)$ above which there are no $\mathbb{Q}_{p}$-points either, so the invariant is constant above any disc contained in this neighborhood.

The algorithm is as follows. First apply a change of coordinates to $\mathbb{P}^{1}$ if necessary, so that the fiber at infinity is smooth. Then find a neighborhood of infinity above which the local invariant is constant. This reduces to considering $t$ in some set of the form $p^{n} \mathbb{Z}_{p}$. For each $t_{0}$ that maps to $\alpha$ in $\mathbb{Q}(\alpha)_{\mathfrak{p}}$ for some place $\mathfrak{p}$ above $p$, find an appropriate disc around $t_{0}$. In doing so we may find that the value of the local invariant corresponding to some $\mathfrak{p}$ is 0 for all $\mathbb{Q}_{p}$-points of $V$. Such $\mathfrak{p}$ can be ignored in the remainder of the computation. If there are points above any of the discs found thus far, compute the corresponding local invariants.

We now divide the remaining region of $\mathbb{P}^{1}\left(\mathbb{Q}_{p}\right)$ into discs and place them in a queue, recording which local invariants we already know to occur. For each disc, we use Lemma 3.29 to try to discover that the local invariant is constant there. If it is, and if the local invariant is already known to occur, the disc may be ignored, as the conclusions do not depend on whether there are rational points there. If it is, and if the local invariant is not already known to occur, we use Lemma 3.30 to test whether there are rational points in that disc. If there are rational points on the disc, we record that the invariant on the disc occurs. The only possible values of the invariant of an element of $\operatorname{Br} V / \operatorname{Br} \mathbb{Q}$ of order 2 are $0, \frac{1}{2} \in \mathbb{Q} / \mathbb{Z}$; if both of these are now known to occur, we are done. On the other hand, if the local invariant is not constant on the disc, we divide it into $p$ smaller discs and add them to the end of the queue. In other words, we perform a breadth-first search. The reason for using breadth-first rather than depth-first search is that local invariants are more likely to be different on relatively distant points of a $p$-adic disc, so breadth-first search will tend to find different invariants more quickly. When the queue is empty we are done and have recorded all values of the local invariant.

Now we show that this algorithm terminates. It could fail to do so only if there is an infinite descending sequence of discs above which there are rational points but in which the local invariant is not seen to be constant. Let $t_{0}$ be the unique point in the intersection of the discs. Then we have shown before that there is a disc around $t_{0}$ on which the local invariant of $\operatorname{cores}(c, t-\alpha)$ is seen to be constant, which is a contradiction, so the algorithm does terminate.

Remark 3.32. This theorem is somewhat special to our situation. If the Azumaya algebra were constructed using fibers of type $I_{2}$, for example, this proof would not apply, and indeed in this case the local invariant associated to a place $\mathfrak{p}$ above $p$ need not be locally constant on $\mathbb{P}^{1}$ around a $t_{0}$ that maps to $\alpha$ in $\mathbb{Q}(\alpha)_{\mathfrak{p}}$. In such situations, it is still possible for the invariant to be locally constant. Sometimes this can be proved by using another Azumaya algebra representing the same Brauer class, as in [11.

Let us now show how to apply this algorithm in our example. 
Proposition 3.33. The Brauer-Manin obstruction blocks the existence of rational points on the K3 surface $V$ corresponding to $(f, \delta)$.

Proof. The surface $V$ is defined by the equations $q_{1}=q_{2}=q_{3}=0,1$ where

$$
\begin{aligned}
q_{1} & =2 x_{1} x_{2}-2 x_{1} x_{3}-2 x_{1} x_{4}+4 x_{1} x_{6}-x_{2}^{2}-2 x_{2} x_{3}+4 x_{2} x_{5} \\
& -4 x_{2} x_{6}+4 x_{3} x_{4}-4 x_{3} x_{5}+2 x_{3} x_{6}-2 x_{4}^{2}+2 x_{4} x_{5} \\
& -6 x_{4} x_{6}-3 x_{5}^{2}+14 x_{5} x_{6}-10 x_{6}^{2}, \\
q_{2} & =2 x_{1}^{2}-5 x_{1} x_{2}+9 x_{1} x_{4}+2 x_{1} x_{5}-7 x_{1} x_{6}+9 x_{2} x_{3}+2 x_{2} x_{4}-7 x_{2} x_{5} \\
& +x_{3}^{2}-7 x_{3} x_{4}+11 x_{3} x_{6}+11 x_{4} x_{5}+8 x_{4} x_{6}+4 x_{5}^{2}+9 x_{5} x_{6}+19 x_{6}^{2}, \\
q_{3} & =21 x_{1}^{2}-44 x_{1} x_{2}-10 x_{1} x_{3}+84 x_{1} x_{4}+18 x_{1} x_{5}-64 x_{1} x_{6}-5 x_{2}^{2} \\
& +84 x_{2} x_{3}+18 x_{2} x_{4}-64 x_{2} x_{5}-38 x_{2} x_{6}+9 x_{3}^{2}-64 x_{3} x_{4}-38 x_{3} x_{5} \\
& +76 x_{3} x_{6}-19 x_{4}^{2}+76 x_{4} x_{5}-58 x_{4} x_{6}-29 x_{5}^{2}-124 x_{5} x_{6}-183 x_{6}^{2} .
\end{aligned}
$$

The primes of bad reduction of $V$ are those dividing the discriminant of $f$, namely $2,3,5$, and those involved in $\delta$, which are again 2,3,5. Each of the fibrations is defined by two alternative pairs of linear forms (see Lemma 2.51 and following remarks). We let

$$
\begin{aligned}
& l_{1}=x_{1}+13 x_{3}+17 x_{4}+68 x_{5}+123 x_{6}, \\
& l_{2}=x_{2}-16 x_{3}-19 x_{4}-84 x_{5}-145 x_{6}, \\
& l_{3}=2 x_{2}-8 x_{3}-8 x_{4}-42 x_{5}-68 x_{6}, \\
& l_{4}=-x_{1}-4 x_{2}+9 x_{3}+5 x_{4}+46 x_{5}+61 x_{6},
\end{aligned}
$$

and then one of the fibrations, which we will denote by $F_{1}$, is given by $\left(l_{1}: l_{2}\right)$ or $\left(l_{3}: l_{4}\right)$, while the other, which will be written $F_{2}$, is given by $\left(l_{1}: l_{3}\right)$ or $\left(l_{2}: l_{4}\right)$. To verify that the two sets of defining equations for each $F_{i}$ give the same map, note that $l_{1} l_{4}-l_{2} l_{3}=-33 q_{1}-74 q_{2}+4 q_{3}$. We have already seen that $F_{1}$ satisfies the hypotheses of Proposition 3.10. now we calculate the local invariant of the associated Azumaya algebra by means of the algorithm described in Theorem 3.31 We will show that this Azumaya algebra has local invariant 1/2 at 2 and 0 at all other primes; the energetic reader may wish to verify that the Azumaya algebra corresponding to $F_{2}$ has local invariant $1 / 2$ at 2,3 , and 5 , and local invariant 0 elsewhere.

The $I_{4}$-fibers of $F_{1}$ lie over the point $\left(129 r^{3}+187 r^{2}+285 r-1469\right) / 449$, where $r^{4}-12 r+13=0$. The fact that this extension is totally complex implies that the local invariant at $\infty$ is 0 , because that local invariant is the sum of local invariants of central simple algebras over $\mathbb{C}$. The components of these fibers are defined over the extension $\mathbb{Q}(r, \sqrt{c})$ where $c=-6 r^{3}-9 r^{2}-12 r+57$ (note that actually $c$ has minimal polynomial $s^{2}-6 s+18$, so $c \in \mathbb{Q}(i)$, as expected from the proof of Theorem 3.14).

\footnotetext{
${ }^{1}$ One can find a linear change of coordinates after which $V$ can be defined by equations with smaller coefficients. We preferred to retain the more natural coordinates.
} 
The fiber at $\infty$ is the vanishing locus of the polynomials

$$
\begin{aligned}
& x_{1}+55 x_{3}+71 x_{4}+290 x_{5}+519 x_{6}, \\
& x_{2}-16 x_{3}-19 x_{4}-84 x_{5}-145 x_{6}, \\
& x_{3}^{2}+414 x_{3} x_{4}-4538 x_{3} x_{5}-5876 x_{3} x_{6}+448 x_{4}^{2}-3796 x_{4} x_{5} \\
& \quad-4200 x_{4} x_{6}-23754 x_{5}^{2}-73732 x_{5} x_{6}-56083 x_{6}^{2}, \\
& 7596 x_{3} x_{4}-83956 x_{3} x_{5}-108804 x_{3} x_{6}+8241 x_{4}^{2}-70306 x_{4} x_{5} \\
& \quad-77950 x_{4} x_{6}-438959 x_{5}^{2}-1362754 x_{5} x_{6}-1036791 x_{6}^{2},
\end{aligned}
$$

and it has good reduction outside $2,3,5,397,449$. This can be checked either by a Groebner-basis computation or by embedding the fiber in $\mathbb{P}^{3}$ as the curve defined by two quadrics $Q_{1}, Q_{2}$ and observing that the curve is nonsingular away from primes dividing the discriminant of $\operatorname{det}\left(t M_{1}+M_{2}\right)$, where $M_{1}$ and $M_{2}$ are the symmetric matrices corresponding to $Q_{1}, Q_{2}$. By Lemma 3.27, the local invariant at every other prime is 0 . We examine these primes in turn.

First we consider $p=2$. We find that there are no rational points in the fibers over $t$ with $v_{2}(t) \leq 1$. In fact, none of the affine patches of the graph of the fibration (see Lemma 3.30) has points mod $2^{3}$ for such $t$. On the other hand, the point $[1: 0: 5: 3: 6: 1]$ modulo 8 can be lifted to a 2 -adic point on the fiber at 0 . Let us show that the local invariant is $1 / 2$ above all $t$ with $v_{2}(t) \geq 2$. Indeed, by Lemma 3.29 it suffices to consider $t=0,4$. There is one prime $\mathfrak{p}$ of $\mathbb{Q}(r)$ above 2 , and it is totally ramified. Therefore it suffices to show that the conics $x^{2}-(t-\alpha) y^{2}-c z^{2}$ are not solvable at $\mathfrak{p}$ for $t=0,4$. In both cases this can be checked modulo $\mathfrak{p}^{5}$.

Next we consider $p=3$. This time we find that there are no rational points in fibers above $t$ with $v_{3}(t) \leq-1$, and that this can be checked modulo $3^{2}$. However, the point $[8: 0: 7: 4: 2: 1]$ modulo 9 can be lifted to a 3 -adic point on the fiber at 4 . Let us show that the local invariant is 0 above all $t$ with $v_{3}(t) \geq 0$. Again, by Lemma 3.29 it suffices to consider $t=0,1,2$. There are two primes of $\mathbb{Q}(r)$ lying above 3 , both unramified of degree 2, and $c$ has valuation 1 at both. On the other hand, $\alpha$ has valuation 0 at both primes and $\alpha$ is not congruent to any integer modulo either prime. Furthermore, $2-\alpha$ is a square at both, while $-\alpha$ and $1-\alpha$ are squares at neither. In particular, it follows from Lemma 3.9 that the local invariant at $t$ is 0 above all $t \in \mathbb{Z}_{3}$.

For $p=5$, the computations are simpler, because $c$ is a square at all primes of $\mathbb{Q}(r)$ lying above 5 . Indeed, there is one ramified prime at which the completion is isomorphic to $\mathbb{Q}_{5}(\sqrt{5})$ and $c$ is congruent to $4 \bmod \sqrt{5}$, and one unramified prime of degree 2 at which $c$ is congruent to $2 \bmod 5$, which is a square in $\mathbb{F}_{25}$. Thus the local invariant is 0 above all $t \in \mathbb{P}^{1}\left(\mathbb{Q}_{5}\right)$.

Finally, we need to consider the primes where $V$ has good reduction but the fiber at infinity has bad reduction, namely 397 and 449 . Note that $c$ is a unit at every place above 397. Also, for every point $P$ whose image $t_{P}$ in $\mathbb{P}^{1}$ is not congruent to $\alpha$ mod any $\mathfrak{p}$ above 397 , the difference $t_{P}-\alpha$ is a unit at all $\mathfrak{p}$ above 397 . Proposition 3.9 then shows that the local invariant of $\left(c, t_{P}-\alpha\right)$ is 0 at all such $\mathfrak{p}$, so that the local invariant of $\operatorname{cores}\left(c, t_{P}-\alpha\right)$ is 0 by Proposition 3.12. Over $\mathbb{F}_{397}$, the $I_{4}$-fibers lie above $(47: 1),(144: 1),(224: 1),(379: 1)$ (the first coordinates are the roots of the minimal polynomial of $\alpha$ in $\mathbb{F}_{397}$ ). Since $V$ has good reduction at 397 , every $\mathbb{F}_{397}$-point on $V$ lifts to a $\mathbb{Q}_{397}$-point, so it suffices to verify that the $\mathbb{F}_{397}$-point 
(246: $16: 98: 0: 1: 0)$ maps to $(0: 1)$ by $F_{1}$. But by Proposition 3.26, it follows that the local invariant at 397 is identically 0 .

To prove that the local invariant at 449 is 0 , it is enough to check that $c$ is a square at all places of $\mathbb{Q}(r)$ above 449; indeed, it is congruent to 204 or 251 at all of these places. Alternatively, it can be verified that the fiber at infinity contains a $\mathbb{Q}_{449}$-point lying above $(246: 105: 375: 347: 1: 0)$. This completes the proof.

Theorem 3.17 now follows by combining Propositions 3.20, 3.21, 3.22, and 3.33,

3.3. The Richelot isogeny. Following a suggestion of Nils Bruin and Victor Flynn, we now study the interaction of the element of $\amalg$ constructed in Theorem 3.17 with the Richelot isogeny on the Jacobian. A Richelot isogeny (cf. [7, Chapter 9) is an isogeny of the Jacobian of a curve of genus 2 to that of another curve whose kernel is a maximal isotropic subgroup of the 2-torsion with respect to the Weil pairing. Given a curve of genus 2 with Weierstrass points $W_{1}, \ldots, W_{6}$, such subgroups consist of 0 and three divisors of the form $W_{i}-W_{j}$ such that no $W_{i}$ appears in more than one of them; since $W_{i}-W_{j}=W_{j}-W_{i}$ in the Jacobian, they correspond to partitions of the Weierstrass points into three pairs. Letting the equation of the curve be $y^{2}=f(x)$, where $\operatorname{deg} f=6$, the Weierstrass points are $\left(\delta_{i}, 0\right)$ where $\delta_{i}$ is a root of $f$, so such partitions correspond to factorizations of $f$ as products $f_{1} f_{2} f_{3}$ of three quadratic factors. The curve whose Jacobian is isogenous is then defined by the equation $y^{2}=c g_{1} g_{2} g_{3}$, where $g_{i}=f_{i+1} f_{i+2}^{\prime}-f_{i+1}^{\prime} f_{i+2}$, indices are read mod 3 , and $c$ is the determinant of the matrix of coefficients of the $f_{i}$ ([7, sect. 9.2). Observe that multiplying one of the $f_{i}$ by a constant $k$ multiplies $c$, $g_{i+1}$, and $g_{i+2}$ by $k$. This is compatible with the evident fact that if the Jacobian of $C$ is isogenous to the Jacobian of $C^{\prime}$, then the Jacobian of the twist of $C$ by $k$ is isogenous to the Jacobian of the twist of $C^{\prime}$ by $k$. In particular, we find that the Jacobian of the curve

$$
C_{t}: y^{2}=t\left(x^{2}+1\right)\left(x^{2}-2 x-1\right)\left(x^{2}+x-1\right)
$$

is isogenous to that of

$$
C_{-t}^{\prime}: y^{2}=-t\left(x^{2}+1\right)\left(x^{2}+2 x-1\right)\left(x^{2}-4 x-1\right)=-t g_{1}(x) g_{2}(x) g_{3}(x)=-t g(x) .
$$

In addition, we observe that there is only one rational Richelot isogeny on each of these curves, because the 2-torsion points that are not rational are defined over extensions of degree 4. A Galois-stable subgroup containing such a point contains four elements other than the identity, so it cannot have order 4 . It follows that the only rational maximal isotropic subgroup is the one made up of the four rational points. From now on, we will denote the Richelot isogeny from $\operatorname{Jac}\left(C_{t}\right)$ to $\operatorname{Jac}\left(C_{-t}^{\prime}\right)$ by $\phi_{t}$, or simply by $\phi$.

First we note that $\amalg\left(\operatorname{Jac}\left(C_{6 n}^{\prime}\right)\right)$ is also nontrivial for $n$ in the set $S$ described in Theorem 3.17

Theorem 3.34. Let $S$ be the set of primes described in Theorem 3.17. Then for all $n$ which are products of elements of $S$, the 2-part of the Tate-Shafarevich group of the Jacobian of the curve $y^{2}=6 n g(x)$ is nontrivial.

Proof. The proof of this theorem is very similar to that of Theorem 3.17 except that one uses $\delta^{\prime}=(3,1+\sqrt{2},(1+\sqrt{5}) / 2) \in \mathbb{Q}[x] /\left(g_{1}(x)\right) \oplus \mathbb{Q}[x] /\left(g_{2}(x)\right) \oplus \mathbb{Q}[x] /\left(g_{3}(x)\right)$. Note that the field of definition of the lines of $V_{g, \delta^{\prime}}$ turns out to be the same as that of $V_{f, \delta}$, because -1 is a square in the splitting field of $g$. 
It is natural to ask whether these systematically-occurring elements of $\amalg$ are in the kernel of the map induced on Tate-Shafarevich groups by $\phi_{-6 n}$. It is not clear whether a general result can be obtained here. We will prove only that they are not in the kernel for the smallest case $n=1$. To do so, we calculate the Selmer groups of these isogenies in the special case $n=1$. See [7, sections 10.2 and 10.4 for the theory of these computations and section 11.4 for a detailed example.

Theorem 3.35. The Selmer groups of the Richelot isogenies on the Jacobians of the curves $y^{2}=-6 f(x)$ and $y^{2}=6 g(x)$ are isomorphic to $(\mathbb{Z} / 2)^{3}$.

Proof. The primes of bad reduction of both $C_{-6}$ and $C_{6}^{\prime}$ are 2,3,5. For both Jacobians, generators of the 2-torsion subgroup map to $(10,2),(2,-2) \in\left(\mathbb{Q}^{*} / \mathbb{Q}^{* 2}\right)^{2}$. From equation $(10.4 .8)$ of [7] we see that $\left|J\left(\mathbb{Q}_{p}\right) / \phi^{\prime}\left(J^{\prime}\left(\mathbb{Q}_{p}\right)\right)\right| \cdot\left|J^{\prime}\left(\mathbb{Q}_{p}\right) / \phi\left(J\left(\mathbb{Q}_{p}\right)\right)\right|$ is equal to $16,4,4,2$ respectively for $p=2,3,5, \infty$. So we have already found generators of $J\left(\mathbb{Q}_{p}\right) / \phi^{\prime}\left(J^{\prime}\left(\mathbb{Q}_{p}\right)\right)$ and $J^{\prime}\left(\mathbb{Q}_{p}\right) / \phi\left(J\left(\mathbb{Q}_{p}\right)\right)$ except in the case $p=2$. We check that there is a $\mathbb{Q}_{2}(\sqrt{6})$-rational point on $C_{-6}$ with $x$-coordinate $3+\sqrt{6}$ whose image is $(10,-2)$ up to 2 -adic squares. Also, there is a $\mathbb{Q}_{2}(\sqrt{6})$-rational point on $C_{6}^{\prime}$ with $x$-coordinate $5+\sqrt{6}$ whose image is $(10,-2)$. These new generators are independent of the image of the 2-torsion points of $C_{-6}$ and $C_{6}^{\prime}$ respectively, so we have found the full Selmer group of the isogeny for both $C$ and $C^{\prime}$ :

\begin{tabular}{|c|c|c|}
\hline$p$ & $C$ & $C^{\prime}$ \\
\hline 2 & $(5,1),(2,2),(1,-1)$ & $(5,1),(2,2),(1,-1)$ \\
3 & $(1,-1),(-1,1)$ & $(1,-1),(-1,1)$ \\
5 & $(5,1),(2,2)$ & $(5,1),(2,2)$ \\
$\infty$ & $(1,-1)$ & $(1,-1)$ \\
\hline
\end{tabular}

It is now straightforward to see that the Selmer groups in both cases are generated by $(5,1),(2,2),(1,-1)$.

It follows immediately that the Selmer groups contain $(1,-1)$, which is not in the image of the 2 -torsion. We now show that $(1,-1)$ is a nonzero element of the Tate-Shafarevich group of the Richelot isogeny, from which it will follow that the elements of $\amalg$ found in Theorem 3.17 and Theorem 3.34 are not in the kernel of the maps $\amalg\left(\operatorname{Jac}\left(C_{-6}\right)\right)[2] \rightarrow \amalg\left(\operatorname{Jac}\left(C_{6}^{\prime}\right)\right)\left[\phi^{\prime}\right]$ and $\amalg\left(\operatorname{Jac}\left(C_{6}^{\prime}\right)\right)[2] \rightarrow \amalg\left(\operatorname{Jac}\left(C_{-6}\right)\right)[\phi]$ induced by the Richelot isogeny for $n=1$.

Theorem 3.36. The element of $\amalg\left(\operatorname{Jac}\left(C_{-6}\right)\right)$ described in Theorem 3.17 is not in the kernel of the map $\amalg\left(\operatorname{Jac}\left(C_{-6}\right)\right)[2] \rightarrow \amalg\left(\operatorname{Jac}\left(C_{6}^{\prime}\right)\right)\left[\phi^{\prime}\right]$ induced by the Richelot isogeny, and similarly for $C_{6}^{\prime}$.

Proof. We will do this only for $C$, the calculations for $C^{\prime}$ being essentially identical. We will first represent the element described in Theorem 3.17 by an explicit cocycle with values in $\operatorname{Jac}\left(C_{-6}\right)[2]$, apply $\phi$, and show that the image is the nontrivial element $(1,-1)$ in $\operatorname{Sel}_{\phi^{\prime}}$.

Recall (Definition 3.13) that we write $\delta_{i}$ for the components of the image of $\delta$ that gives the nontrivial element of $\amalg\left(\operatorname{Jac}\left(C_{-6 n}\right)\right)$ under a fixed isomorphism $A_{f} \otimes \overline{\mathbb{Q}} \rightarrow \oplus_{1}^{6} \overline{\mathbb{Q}}$ in which $\overline{\mathbb{Q}}[X] /\left(f_{j}\right)$ corresponds to components $2 j-1$ and $2 j$. Given $\sigma \in \operatorname{Gal}(\overline{\mathbb{Q}} / \mathbb{Q})$, write $s$ for the permutation of $\{1,2, \ldots, 6\}$ induced by $\sigma$ on the $\delta_{j}$. By remarks in the proof of [16, Proposition 2.2 and in [16, section 2.5, we can write the element of $\amalg$ as a cocycle with values in $\mu_{2}\left(A_{f} \otimes \overline{\mathbb{Q}}\right) / \pm 1$. As in the discussion preceding Lemma 2.10 , we identify $A_{f} \otimes \overline{\mathbb{Q}}$ with $\oplus_{1}^{6} \overline{\mathbb{Q}}$ with Galois acting 
by ${ }^{\sigma}\left(a_{1}, \ldots, a_{6}\right)=\left({ }^{\sigma} a_{s^{-1}(1)}, \ldots,{ }^{\sigma} a_{s^{-1}(6)}\right)$. Indeed, the cocycle corresponding to the element of $\amalg$ constructed in Theorem 3.34 is the one that takes $\sigma \in \operatorname{Gal}(\overline{\mathbb{Q}} / \mathbb{Q})$ to ${ }^{\sigma} \alpha / \alpha$, where

$\alpha=\left(\sqrt{\left(\delta_{1} \delta_{j}\right)}\right)_{j=1}^{6}=\sqrt{(1,1,-3(1+\sqrt{2}),-3(1-\sqrt{2}), 3(1+\sqrt{5}) / 2,3(1-\sqrt{5}) / 2)}$.

In particular, this cocycle, which we will denote $z_{\alpha}$, factors through $\operatorname{Gal}(F / \mathbb{Q})$, where $F$ is as in Theorem 3.17

Now let us write $z_{\alpha}$ as a cocycle with values in $J[2]$. Following the description in [16, section 2.5, we see that $\left(r_{i}\right) \in \mu_{2}\left(L^{\prime}\right) / \pm 1$ corresponds to a 2-torsion point $T$ on the Jacobian such that either $e\left(T,\left(\delta_{j}, 0\right)-\left(\delta_{1}, 0\right)\right)$ is equal to $r_{j}$ for all $j$ from 1 to 6 or it is equal to $-r_{j}$ for all $j$ from 1 to 6 , where $e$ is the Weil pairing. The rational 2-torsion divisors are 0 and $\left(\delta_{2 k}, 0\right)-\left(\delta_{2 k-1}, 0\right)$ for $k=1,2,3$. Since two 2 -torsion divisors of the form $\left(\delta_{i}, 0\right)-\left(\delta_{j}, 0\right)$ have Weil pairing 1 or -1 depending on whether the number of points appearing in both counted with multiplicity is even or odd, the rational 2-torsion points correspond to the following elements of $\mu_{2}\left(L^{\prime}\right) / \pm 1$ :

$$
(1,1,1,1,1,1), \quad(1,1,-1,-1,1,1), \quad(1,1,1,1,-1,-1), \quad(1,1,-1,-1,-1,-1) .
$$

We claim that the image of $z_{\alpha}(\sigma)$ under the pairing with the points $\left(\delta_{j}, 0\right)-\left(\delta_{1}, 0\right)$ is one of these sequences corresponding to an element of the kernel of the Richelot isogeny if and only if $\sigma$ fixes a square root $i$ of -1 . Indeed, it is clear that the first two components of $z_{\alpha}(\sigma)$ are always 1 , while the product of components 3 and 4 is 1 if and only if $\sigma$ fixes a square root of $\delta_{3} \delta_{4}=-9$, and similarly for components 5 and 6 .

These points are the kernel of the Richelot isogeny, which therefore maps $z_{\alpha}$ to a cocycle that factors through $\mathbb{Q}(i)$ and that takes the nontrivial element of this Galois group to the rational 2-torsion point arising from the factor $g_{1}$. This corresponds to the element $(1,-1)$ of the Selmer group of $\phi^{\prime}$. To see this, note that $(1, i)$ is a square root of $(1,-1)$, and the action of $\sigma$ multiplies $(1, i)$ by $(1,1)$ if $\sigma$ fixes $i$ and by $(1,-1)$ otherwise. But the image of the 2 -torsion point coming from $g_{1}$ under the $\phi^{\prime}$-Weil pairing is $(1,-1)$, because the pullback of this point pairs trivially with the point coming from the factor $f_{1}$ on $J$ and nontrivially with the point coming from $f_{2}$. As a result, the image of $z_{\alpha}$ under the Richelot isogeny corresponds to the cocycle $\sigma \rightarrow^{\sigma}(1, i) /(1, i)$.

Recall from Definition 3.18 and Proposition 3.19 that the full 2-descent map $\Delta_{C^{\prime}}$ is a map from $\operatorname{Jac}\left(C^{\prime}\right)(\mathbb{Q})$ to $\mathbb{Q}[X] /(g)$. Composing $\Delta_{C^{\prime}}$ with an isomorphism from $\mathbb{Q}[X] /(g)$ to $\bigoplus_{i=1}^{3} \mathbb{Q}[X] /\left(g_{i}\right)$ obtained from the Chinese Remainder Theorem, we see that the map takes a point $\left(x_{0}, y_{0}\right)$ to $\left(x_{0}-\rho_{i}\right)_{i=1}^{3}$; in other words, its components are defined by the functions $x-\rho_{i}$. We remark that $g_{1}=N\left(x-\rho_{1}\right)$ and $g_{2}=N\left(x-\rho_{2}\right)$. It follows that if $P$ is a point of $\operatorname{Jac}\left(C_{6}^{\prime}\right)$, then the image of $P$ under $\delta_{R, C^{\prime}}$, the descent map for the Richelot isogeny, is the norm of the first two components of its image under $\Delta_{C^{\prime}}$.

However, using MAGMA's TwoSelmerGroup command to compute the fake Selmer group, it is easy to verify that the norm map from the fake Selmer group for full 2-descent to the Selmer group of the Richelot isogeny is an isomorphism for $C_{6}^{\prime}$. It follows that the image of an element of $\amalg\left(C_{6}^{\prime}\right)$ cannot be the image of a rational 
divisor, so $(1,-1)$ belongs to the Tate-Shafarevich group of the Richelot isogeny on $C_{6}^{\prime}$.

Remark 3.37. This proof applies to any twist $C_{6 n}^{\prime}$, where $n$ is a product of elements of the set $S$ described in Theorem 3.34, for which we know that no rational point on the Jacobian of $C_{6 n}^{\prime}$ maps by $\delta_{R}$ to $(1,-1)$. However, there is no reason to expect this to be true in general; if the rank is large, it is very unlikely that the fake Selmer group for multiplication by 2 would be isomorphic to the Selmer group of the Richelot isogeny, and the proof would fail.

\section{ACKNOWLEDGMENTS}

We thank Michael Stoll for suggesting this project to us, Nils Bruin and Victor Flynn for helpful suggestions and explanations, William Stein for letting us use his computers, CRM in Montreal and MSRI for their hospitality and financial support, those who have supported us through their grants, and the MAGMA group for developing their software. Also, the first author thanks the Nuffield Foundation for funding his research with a grant in their Awards to Newly Appointed Lecturers program, which supported him at CRM in the fall of 2005, and the University of Waterloo. The second author also thanks PIMS, Simon Fraser University, the University of British Columbia, and Universidad de los Andes.

\section{REFERENCES}

[1] W. Barth, C. Peters, and A. van de Ven, Compact Complex Surfaces, Ergebnisse der Mathematik und ihrer Grenzgebiete, 3. Folge, Band 4, Springer-Verlag, 1984. MR749574 (86c:32026)

[2] E. Bombieri and D. Mumford, Enriques' classification of surfaces in char. p, II. Complex Analysis and Algebraic Geometry - Collection of papers dedicated to K. Kodaira, W.L. Baily and T. Shioda, eds. Iwanami and Cambridge Univ. Press (1977), 23-42. MR0491719 (58:10922a)

[3] M. Bright, Brauer Groups of Diagonal Quartic Surfaces, J. Symbolic Computation 41 (2006), 544-558. MR2209163 (2007h:14022)

[4] M. Bright, Computations on Diagonal Quartic Surfaces, Unpublished Ph.D. dissertation, Cambridge University, 2002, available at www.boojum.org.uk.

[5] M. Bright and H. P. F. Swinnerton-Dyer, Computing the Brauer-Manin obstructions, Math. Proc. Camb. Phil. Soc. 137 (2004), 1-16. MR2075039 (2005c:11081)

[6] N. Bruin and E. V. Flynn, Exhibiting SHA[2] on hyperelliptic Jacobians, J. Number Theory 118 (2006), 266-291. MR2225283 (2006m:11091)

[7] J. W. S. Cassels and E. V. Flynn, Prolegomena to a Middlebrow Arithmetic of Curves of Genus 2, LMS Lecture Notes 230, Cambridge University Press (1996). MR1406090 (97i:11071)

[8] P. Deligne, Cohomologie des intersections complètes, Exposé XI in Groupes de monodromie en géométrie algébrique (SGA 7 II), Lecture Notes in Math. 340, Springer, Berlin, 1973.

[9] R. Hartshorne, Algebraic Geometry, GTM 52, Springer-Verlag, New York, 1977. MR0463157 $(57: 3116)$

[10] R. Hartshorne, Equivalence relations of algebraic cycles and subvarieties of small codimension, Algebraic Geometry, Arcata 1974, Amer. Math. Soc. Proc. Symp. Pure Math. 29 (1975), 129-164. MR0369359 (51:5592)

[11] A. Logan, The Brauer-Manin obstruction on del Pezzo surfaces of degree 2 branched along a plane section of a Kummer surface, Math. Proc. Camb. Phil. Soc., to appear.

[12] Yu. Manin, Cubic forms: algebra, geometry, arithmetic, North-Holland Mathematical Library 4, North-Holland, Amsterdam, 1986 (2nd edition, translated by M. Hazewinkel). MR833513 (87d:11037)

[13] J. S. Milne, Étale Cohomology, Princeton Mathematical Series 33, Princeton University Press, Princeton, 1980. MR559531 (81j:14002) 
[14] D.R. Morrison, On K3 surfaces with large Picard number, Invent. Math. 75 (1984), 105-121. MR.728142 (85j:14071)

[15] B. Poonen and M. Stoll, The Cassels-Tate pairing on polarized abelian varieties, Ann. of Math. (2) 150 (1999), no. 3, 1109-1149. MR1740984 (2000m:11048)

[16] E.F. Schaefer, Computing a Selmer group of a Jacobian using functions on the curve, Math. Ann. 310 (1998), 447-471. MR1612262(99h:11063)

[17] J.-P. Serre, Local Fields, GTM 67, Springer-Verlag, New-York, 1979. MR554237|(82e:12016)

[18] T. Shioda, Algebraic cycles on certain K3 surfaces in characteristic p, Manifolds-Tokyo 1973 (Proc. Internat. Conf., Tokyo, 1973), Univ. Tokyo Press, Tokyo (1975), 357-364. MR0435084 $(55: 8046)$

[19] A. Skorobogatov, Torsors and rational points, Cambridge Tracts in Mathematics 144. Cambridge University Press, Cambridge, 2001. MR1845760 (2002d:14032)

[20] M. Stoll, Implementing 2-descent for Jacobians of hyperelliptic curves, Acta Arith. 98 (2001), no. 3, 245-277. MR:1829626 (2002b:11089)

[21] R. van Luijk, An elliptic K3 surface associated to Heron triangles, Journal of Number Theory, 123 (2007), 92-119. MR 2295433 (2007k:14077)

[22] R. van Luijk, K3 surfaces with Picard number one and infinitely many rational points, J. Algebra Number Theory 1 (2007), no. 1, 1-15. MR2322921

[23] Electronic resources, available at http://oldweb.cecm.sfu.ca/ ${ }^{\sim}$ rluijk/ShaK3/.

Department of Pure Mathematics, University of Waterloo, Waterloo, On, Canada, N2L $3 \mathrm{G} 1$

E-mail address: a5logan@math.uwaterloo.ca

Department of Mathematics, Simon Fraser University, Burnaby, BC, Canada, V5A $1 \mathrm{~S} 6$

E-mail address: rmluijk@gmail.com 